\title{
Effects of Selective Attention on Sensory Processing of Visual Motion
}

\author{
Dissertation \\ zur Erlangung des Doktorgrades \\ der Mathematisch-Naturwissenschaftlichen Fakultäten \\ der Georg-August Universität zu Göttingen
}

vorgelegt von

Laura Busse, M.Sc. (Neural \& Behavioral Sciences) aus Bergisch-Gladbach

Göttingen 2006 
D 7

Referent: Prof. Dr. Stefan Treue

Korreferent: Prof. Dr. Julia Fischer

Tag der mündlichen Prüfung: 31.10.2006 


\section{Acknowledgments}

The work included in this thesis would never have been possible without ample expert support. First of all, I would like to thank Stefan Treue for giving me the possibility to work in his laboratory and to study under his supervision. I am very grateful for the numerous opportunities he offered me during the course of the years and for the independence with which I could conduct this research. Christoph Nothdurft and Fred Wolf, both members of my PhD committee, have always offered important advice and constructive criticism, which I would like to offer my thanks for. I also thank Julia Fischer for her kind support in evaluating this thesis.

For the electrophysiological part of this work, thanks are owed to Dirk Prüsse from whom I always received expert support regarding all questions of animal care-taking. A special note of gratitude also goes to Leonore Burchardt, Daniel Kaping, and Pinar Boyraz who looked after Tiny and Carlos when I was not in Göttingen. Florian Pieper's knowledge regarding the recording setup and system was indispensable for this work, and I would like to thank him once again for his help. I am also very happy to thank Sabine Stuber for dealing with all administrative issues and Ralf Brockhausen for his support in computer-related questions.

I have had the good fortune to talk about my work with Tzvetomir Tzvetanov, Florian Pieper, Thilo Womelsdorf, Christine Tillmann, and Alexander Gail, who have all provided important insights during our discussions. Of special importance, I would like to thank Pinar Boyraz for making the lab an enjoyable and productive place to be - thanks for sharing an office with me during all these years.

I thank my parents, Heinrich and Anne, for their continuous interest, encouragement and support, and my sister, Barbara, for her good humor. It is always very pleasant to be home. Special thanks go to my friends for their companionship: Silke, Nils, Gundula, Maria, Jana, Conni, Juliane, Jürgen, Meike.

And, Steffen, thank you for the richness of life. 


\section{Contents}

1 Introduction 1

1.1 Motion processing in the primate visual system $\ldots \ldots \ldots \ldots 2$

1.1.1 Visual areas involved in motion processing . . . . . . . . . . 2

1.1.2 Anatomical and functional properties of macaque area MT . . . . . . . 2

1.1.3 Area MT and the perception of motion . . . . . . . . . . . . 3

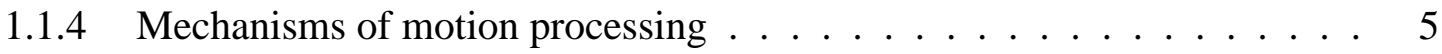

1.2 Cognitive influences on sensory visual processing . . . . . . . . 7

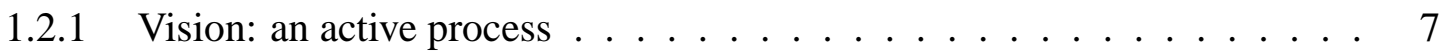

1.2.2 Mechanisms of selective visual attention . . . . . . . . . . . 8

1.2.3 Temporal dynamics of attention $\ldots \ldots \ldots \ldots \ldots$

2 Original articles and manuscripts $\quad 11$

2.1 Temporal dynamics of attentional modulation . . . . . . . . . . . . 12

2.2 Time course of shifting visual attention $\ldots \ldots \ldots \ldots \ldots$

2.3 Exogenous cueing of motion processing $\ldots \ldots \ldots \ldots$

2.4 Attentional effects on perceptual tuning curves $\ldots \ldots \ldots \ldots \ldots$

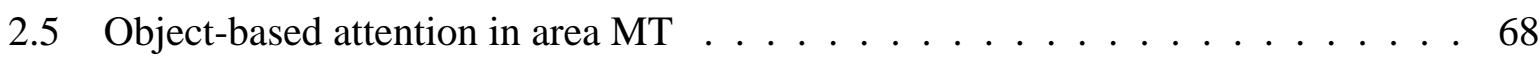

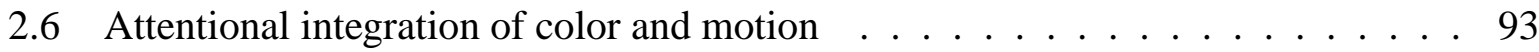

2.7 Benefits of signal anticipation $\ldots \ldots \ldots \ldots$

$\begin{array}{llr}3 & \text { Summary } & 127\end{array}$

$\begin{array}{lr}\text { Bibliography } & 129\end{array}$

$\begin{array}{lr}\text { Curriculum Vitae } & 137\end{array}$ 


\section{Chapter 1}

\section{Introduction}

The topic of this thesis is centered on the question of how cognition influences the processing of sensory information. The main part of this work consists of seven research articles and manuscripts. Three of these articles are published in scientific journals, one manuscript is currently under review, and the remaining three are prepared for submission.

To study the cognitive influences on the processing of sensory information our research group uses, as a model system, the highly-developed ability of primates to process visual motion. In this work, I have combined two different, but complementary techniques: One the one hand, I have performed extracellular recordings in the cortical motion-sensitive middle temporal area (MT) of macaque monkeys to investigate how attention changes the neural representation of motion information. On the other hand, I have used psychophysical methods to study how human motion perception is altered by various cognitive factors, such as attention or expectation.

This chapter is divided into two parts. The first part gives a brief overview of the processing of motion in the primate visual system, emphasizing the role of area MT. The second part reviews how the processing of sensory information is shaped by higher-level, cognitive factors. Here, I mainly focus on selective attention, which is one of the most important top-down mechanisms modulating the processing of sensory information.

The third and main part of this thesis is composed of original research articles and manuscripts. The objective of the individual experiments and the major findings will be briefly summarized in separate subsections preceding each manuscript. 


\subsection{Motion processing in the primate visual system}

\subsubsection{Visual areas involved in motion processing}

The accurate representation of visual motion in the environment is one of the most important tasks of the visual system because motion signals can convey important information about predators and prey, about the spatial layout and other properties of objects in the scene, and about the self-motion of the organism.

Given this importance, it is not surprising that the primate brain contains specialized neurons and even entire visual areas devoted to the processing of visual motion. In primates, the earliest processing stage containing neurons sensitive to motion is the primary visual cortex (V1), where about $25 \%$ of the neurons exhibit direction selectivity (Hubel and Wiesel, 1968), i.e. they respond vigorously to one direction of motion and show little or no responses to the opposite direction. In V1, direction-selective neurons are confined to the upper layers of layer 4 (4a, 4b, $4 \mathrm{c} \alpha$ ) and layer 6 (Hawken et al., 1988). Of these, neurons in layers $4 \mathrm{~b}$ and $4 \mathrm{c} \alpha$ project directly, or via V2, to the middle temporal area (MT/V5), where direction selective neurons are extraordinarily preponderant and where directional selectivity is strong (Dubner and Zeki, 1971). Several brain areas beyond MT, e.g. MST and VIP, respond to complex forms of motion, like rotation, expansion, contraction, and optic flow. In humans, brain imaging studies have revealed a network of brain areas responsive to motion (Culham et al., 2001). The best studied region among these is the MT+ complex, which is a homologue of macaque MT, MST, and adjacent motion selective cortex, located at the junction of the inferior temporal sulcus and lateral occipital sulcus (Zeki et al., 1991).

\subsubsection{Anatomical and functional properties of macaque area MT}

In the macaque, area MT is one of the most studied parts of the visual cortex. MT is located in the upper, lateral bank of the superior temporal sulcus, and it is anatomically characterized by dense myelination and direct reciprocal connections with area V1 (Van Essen et al., 1981).

In addition to the very important input from area V1, MT receives ascending input from several other cortical and subcortical structures, including areas V2, V3, the koniocellular neurons of the LGN, the superior colliculus, and the pulvinar. MT is also connected to several areas of the superior temporal sulcus (e.g. MST, FST) and parietal sulcus (e.g. VIP). In addition, it has extensive connections with frontal lobe areas (e.g. FEF). This network of connections identifies MT as one of the major areas of the dorsal processing stream projecting to structures that are implicated in the analysis of optic flow and planning of upcoming movements (Britten, 2004). 
Area MT has fairly orderly arranged maps for several stimulus characteristics. First, MT contains a retinotopic representation of the contralateral visual space, i.e. neighboring neurons in MT represent approximately neighboring regions of the visual field. The fovea is typically represented laterally, the periphery medially; the representation of the upper contralateral quadrant representation is located anterior, and that of the lower quadrant posterior (Gattass and Gross, 1981). Second, directionality in MT is organized in a columnar pattern, such that neurons across the cortical layers within a column prefer the same direction of motion while direction preference systematically changes across columns. These changes can either be gradual or abrupt with preferred direction changing by 180 deg (Dubner and Zeki, 1971; Albright et al., 1984; Geesaman et al., 1997). Third, there is a columnar organization in terms of binocular disparity tuning that, within disparity tuned patches of MT, changes gradually across columns (DeAngelis and Newsome, 1999). Finally, a clustering of speed preferences, without columnar organization, has also been reported for area MT (Liu and Newsome, 2003).

Receptive fields (RFs) of MT neurons have several properties that are directly relevant to the experiments presented below. A RF can be defined as the region in visual space from which a stimulus can activate the neuron under study. RFs in area MT are $\sim 10$ times larger than those in area $\mathrm{V} 1$, with their diameter being related to eccentricity by a factor of $\sim 0.8$ (Maunsell and Van Essen, 1983). About 90\% of the neurons in area MT show strong selectivity for direction of motion, i.e. they respond vigorously to a particular direction of motion ("preferred direction") and much less to the opposite direction ("null direction" or "antipreferred direction"). The direction tuning curves, i.e. the average responses plotted as a function of the stimulus direction, can typically be very well fitted by a Gaussian function. The directional selectivity, i.e. the width of the tuning curves, generally ranges between 40-60 deg (Albright, 1984; Snowden et al., 1992; Britten, 2004). Furthermore, MT neurons are speed-tuned, preferring speeds between 5-30 deg/s (Albright, 1984; Britten, 2004).

\subsubsection{Area MT and the perception of motion}

The anatomical and functional properties of neurons in area MT make this area an ideal candidate for the analysis of visual motion. Thus, the relationship between activity in area MT and the perception of motion has been addressed by various approaches (Parker and Newsome, 1998).

Lesion studies in area MT showed a selective impairment of motion processing, demonstrating a first link between activity in MT and motion perception. It has been found that, after lesioning MT and the surrounding cortex, psychophysical thresholds were elevated in motion detection and discrimination tasks (Newsome and Pare, 1988; Orban et al., 1995; Pasternak and Merigan, 1994; Lauwers et al., 2000), while contrast thresholds (Newsome and Pare, 1988) and 
detection thresholds for color and texture differences (Schiller, 1993) were largely unaffected. It should be noted though, that thresholds can substantially recover within a few weeks (Newsome and Pare, 1988), suggesting reorganization across areas or within area MT.

A further finding supporting a prominent role of area MT in motion perception has been the similarity between the sensitivity of individual neurons in area MT and the psychophysical sensitivity of the observer (Newsome et al., 1989; Britten et al., 1992). Newsome et al. (1989) recorded the activity of individual MT neurons while the monkeys reported the direction of motion in a random dot display. The difficulty of this task could be varied by manipulating the percentage of dots that moved coherently, while the remaining dots moved in random directions. Detection thresholds for individual neurons were computed using methods from signal detection theory (Tanner and Swets, 1954) and could be directly compared to the behavioral thresholds of the observers. The authors found that single neurons were at least as sensitive as the monkey himself, indicating that the directional signal could be carried by a relatively small population of neurons (alternatively, a larger population might be involved in the decision if noise in the responses of MT neurons was correlated or relatively insensitive neurons were included in the population code (Shadlen et al., 1996)). It should be noted, thought, that the interpretation of this finding is complicated, because the motion signal was always presented for a fixed duration of 2 seconds (see also, Cook and Maunsell, 2002). While the neuronal activity integrated across this time entered the analysis, it is not clear which temporal strategy the monkey adopted, i.e. whether he perceptually integrated the motion information during the entire time window or whether he made his decision before the end of the period. In addition, several studies comparing behavioral and neuronal sensitivity in fine direction (Purushothaman and Bradley, 2005) and speed (Liu and Newsome, 2005) discrimination tasks report that MT neurons are much less sensitive than the observer. These findings indicate that the relationship between behavioral and neuronal sensitivity might depend on the task and hence on the computational role the neuron plays in the decision process (Born and Bradley, 2005).

Furthermore, reliable biases of motion perception have been reported in microstimulation experiments (Salzman et al., 1990). Stimulating clusters of MT neurons preferring a particular direction leads to an increase in the proportion of behavioral choices in favor of the direction preferred by the stimulated neurons, indicating an induced perceptual bias towards that direction. These results indicate that MT is directly and causally involved in the monkey's perceptual decision about the stimulus direction. Similar results have been obtained for judgments of stereoscopic depth (DeAngelis et al., 1998) and speed (Liu and Newsome, 2005).

Finally, significant trial-by-trial correlations between the firing rate of individual MT neurons and the choice of the monkey argue for an important role of MT in the perception of motion (Britten et al., 1996). When presenting the monkey with $0 \%$ coherent motion in a direction 
detection task, the upcoming behavioral choice of the monkey could be reliably predicted using an ideal-observer analysis of the firing rate of single MT neurons (choice probability). Together, these experiments demonstrate that area MT plays an integral role in motion perception.

\subsubsection{Mechanisms of motion processing}

The physiological mechanisms underlying the computation of visual motion are a topic of current debate. To begin, it should be noted that the perception of motion is a visual inference because motion information reaching our photoreceptors is encoded as a sequence of discrete two-dimensional patterns of changing intensities of light (Wandell, 1995). This implies that, first, if an object moves through the scene, the sequential activation of the photoreceptors and retinal ganglion cells covering the representation of the motion path has to constitute the basis for any subsequent direction and speed judgment (see also, Frechette et al., 2005). Hence, appropriately timed sequences of light flashes should evoke a perception of continuous motion, a phenomenon called apparent motion. Second, since receptive fields in early visual processing stages are rather small, neurons are confronted with the aperture problem when an object larger than their receptive field moves across the visual field. The aperture problem is the inability to measure the motion along a constant spatial direction; the only direction of motion a detector can signal is perpendicular to any edge that crosses its receptive field.

Computational models in combination with recent advances in RF mapping techniques have provided insights into the mechanisms of motion processing and their implementation in the brain. Models of image motion formation have proposed that motion information is associated with energy in a particular space-time orientation (Adelson and Bergen, 1985). In these models, the detection of motion is based on neurons with an appropriate spatiotemporal impulse response function. Here, the spatial impulse response function describes the weighting of light information across space, and the temporal response function describes how information from the past is summed to produce the response at the present moment (Dayan and Abbott, 2001). Linear approximations of a neuron's spatial and temporal impulse response functions can be experimentally determined in a robust and efficient way using white-noise analysis (Chichilnisky, 2001). If spatial and temporal impulse response functions can simply be multiplied to yield a neuron's spatio-temporal RF, the RF is called space-time separable. Space-time separable RFs strongly respond to moving stimuli of the appropriate speed, however, they cannot distinguish between opposite directions of motion. Such space-time separable RFs can be found for some simple cells in area V1 (DeAngelis et al., 1995). In contrast, accounting for direction selectivity requires space-time inseparable RFs, i.e. RFs which cannot be described by a product of the spatial and temporal impulse response function. Space-time inseparable RFs are also found for V1 
simple cells and can be modeled by adding two or more space-time separable RFs with different spatial and temporal characteristics (Watson and Ahumada Jr., 1983). Finally, direction-selective spatial-phase invariant responses, as found for V1 complex cells, can be obtained by squaring and summing the output of four space-time inseparable RFs that are 90 deg out of phase (energy model) (Adelson and Bergen, 1985; Dayan and Abbott, 2001).

It has been proposed that area MT inherits most of the raw direction and speed tuning already found in $\mathrm{V} 1$, yet, it might play a fundamental role in computing the motion of whole objects or patterns (Born and Bradley, 2005). Because of their small RFs, neurons in V1 are severely faced with the aperture problem and thus can only report velocities orthogonal to each local contour of a moving object composed of many different orientations (velocity is the vector representation of the direction and speed). Combining at least two samples of local motion is sufficient, in theory, to compute the object motion; the geometrical solution to this problem is called the intersection of constraints (IOC). Movshon et al. (1985) have used plaid stimuli, i.e. two superimposed gratings moving in different directions, to test whether neurons in MT can signal the motion direction predicted by the IOC solution. The authors found that $\sim 25 \%$ of MT cells responded to the pattern motion (which is also perceived by human observers (Adelson and Movshon, 1982)) and termed these cells pattern direction-selective cells. About $40 \%$ of the recorded MT neurons signalled the component directions of the individual gratings (component direction-selective cells), and the remaining neurons were intermediate. Importantly, none of the V1 neurons were selective to the pattern motion (but see, Guo et al., 2004; Tinsley et al., 2003). This finding led the authors to propose a two-stage model, in which the first stage (V1) computes local motion components which are then integrated by the second stage (MT) establishing the velocity of motion of the entire pattern. This idea has been formalized in a computational model of MT physiology (F-plane model, Simoncelli and Heeger, 1998). The model is based on the notion that the Fourier transform of any translational pattern lies on a plane in frequency space (Watson and Ahumada Jr., 1983). To compute pattern velocity, the model assumes that the output of V1 complex cells tuned for spatio-temporal orientation is weighted and summed over this plane in frequency space. This planar summation for velocity selectivity is an implementation of the IOC solution.

In summary, anatomical, functional, and computational properties of area MT, as well as the link between neuronal activity and perception of motion, make this visual area a prime candidate to investigate cognitive influences on processing of motion information. 


\subsection{Cognitive influences on sensory visual processing}

\subsubsection{Vision: an active process}

From the earliest stages of the visual processing pathway on, vision does not yield a 1:1 representation of the world but is instead characterized by the enhancement of behaviorally important information. On the one hand, an enhanced representation of the relevant information is achieved by mechanisms built into the organizational principles of the visual system. One example for such mechanisms is the primate fovea, the part of the retina which is most densely populated by cone photoreceptors and, hence, the area of highest visual acuity. In contrast, information falling on the more peripheral part of the retina is sampled much more coarsely. To make efficient use of this retinal patch of exquisite visual acuity, primates make about 3-5 saccades per second, thereby bringing the most relevant aspects of the visual scene onto the most sensitive part of the retina. Likewise, lateral inhibition and the organization of center-surround RFs in the retina constitute very early-level mechanisms mediating enhancement of luminance boundaries. Besides these hard-wired mechanisms of signal enhancement, higher level cognitive processes can also substantially modulate the processing of visual information.

The most prominent of such higher level cognitive factors is attention (Desimone and Duncan, 1995; Kastner and Ungerleider, 2000; Treue, 2001), which can be defined as the selective modulation of visual information according to behavioral relevance. Different forms of visual attention have been described. First, spatial attention refers to a situation in which attentional resources are focused on a location in space. Using a variety of brain activity measures it has been demonstrated that neurons respond more strongly when attention is directed to a location corresponding to their retinotopic position compared to when attention is directed somewhere else (Brefczynski and DeYoe, 1999; Luck et al., 1997; Treue and Maunsell, 1996). Second, attention can also be oriented to non-spatial stimulus features, like a particular color or direction of motion (Maunsell and Treue, 2006). This form of attention might be especially useful in situations in which a particular stimulus has to be found in a cluttered visual scene (visual search task, Treisman and Gelade, 1980). The neuronal correlate of feature-based attention has been shown to consist of a selective response enhancement of neurons preferring the attended feature (Treue and Martínez-Trujillo, 1999). Importantly, this enhancement occurs independently of spatial location (Treue and Martínez-Trujillo, 1999; Sàenz et al., 2002, 2003). Third, attention can also be object-based in the sense that attending to a single feature of an object will lead to a response enhancement of neurons preferring any feature constituting the object, even if irrelevant to the behavioral task (O'Craven et al., 1999). Recently, it has been shown that the modulation of responses to irrelevant features of an attended object is not spatially specific but "spreads" to 
all locations in visual space (Melcher et al., 2005; Boynton et al., 2006).

\subsubsection{Mechanisms of selective visual attention}

After having introduced several concepts of attention, I will briefly review three key lines of research concerning the mechanisms of visual attention: 1. How does attention change neuronal tuning curves? 2. Does attention affect the spatial RF profile? 3. How does attention change the contrast response function?

While attention enhances neuronal responses it does not seem to alter the sensitivity of individual neurons. In the case of spatial attention, directing attention to the stimulus inside the RF compared to any other spatial location multiplicatively increases the responses of single neurons to all stimuli along their tuning curve, without broadening or sharpening their selectivity (i.e., tuning width). Such gain modulation has been demonstrated for individual neurons in both areas V4 (McAdams and Maunsell, 1999) and MT (Treue and Martínez-Trujillo, 1999). Similarly, attending to the preferred feature of a neuron at a location distant from its RF (featurebased attention) also proportionally scales its tuning curve without changing its width (Treue and Martínez-Trujillo, 1999). The observed effects in area V4 could be best explained by a response gain model, which states that only stimulus-driven, evoked activity, but not undriven, spontaneous activity, is modulated by attention (McAdams and Maunsell, 1999). Because the study on MT direction tuning did not discuss modulations of undriven activity, these data cannot distinguish between response gain or activity gain models, which propose that also the undriven activity is modulated by attention. The effects of attention on population tuning curves have not been directly investigated, so far; however, by appropriately combining the single-unit data it has been suggested that spatial attention exerts multiplicative influences also on the population level (McAdams and Maunsell, 1999). In contrast, a non-multiplicative modulation of population tuning curves has been proposed for feature-based attention, with an enhancement of neurons preferring the attended feature and a suppression of neurons preferring the opposite feature (Martínez-Trujillo and Treue, 2004).

Attention has been shown to modulate spatial RF properties. Since the earliest investigations of attentional modulation it has reliably been observed that attention to one of multiple stimuli within a RF makes the neuronal responses more similar to the responses when the attended stimulus is presented alone (Moran and Desimone, 1985), suggesting that receptive field centers shift towards the attended stimulus and/or contract around it. Two studies have directly investigated this question and found reliable shifts of RF centers toward the attended stimulus in areas V4 (Connor et al., 1997) and MT (Womelsdorf et al., 2006), and very moderate shrinkage (Womelsdorf et al., 2006). A computational study of the mechanism underlying these changes in RF 
profile provides evidence that RF shifts in secondary visual areas such as V4 or MT can be accounted for by a multiplicative scaling of tuning curves in early visual areas, such as V1 or V2 (Compte and Wang, 2006).

Several studies have related the effects of attention to increases in stimulus contrast. First, stimulus contrast has long been known to affect neuronal responses at all levels of visual processing. This makes effects of increased stimulus contrast a very good analogy to effects of attention, which have been observed in many stages of visual processing and as early as the LGN (McAlonan et al., 2006; O'Connor et al., 2002). Second, increasing the stimulus contrast multiplicatively scales neuronal tuning curves, an effect reminiscent of the effects of attention (see above). Reynolds et al. (2000) determined the attentional modulation in area V4 for a range of stimulus contrasts and found that the most prominent effects occurred for lower and intermediate stimulus contrasts. This finding is consistent with a leftwards shift of the contrast response function (contrast gain model). Later, this result was confirmed for area MT in which attentional modulation was measured while an irrelevant stimulus inside the RF varied in contrast (Martínez-Trujillo and Treue, 2002). While the evidence has so far pointed towards a nonlinear interaction between contrast and attention, a recent study by Williford and Maunsell (2006) in area V4 casts doubt on the generality of this finding. The authors report that the effects of attention on responses to stimuli of various contrasts could be very well described by either contrast gain, response gain, or activity gain models, with a slight superiority of response gain or activity gain models. Since the previous studies did not compare the explanatory power of contrast gain and response gain models (Reynolds et al., 2000) or obtained good model fits for both the contrast gain and the response gain model (Martínez-Trujillo and Treue, 2002), response or activity gain models cannot definitively be ruled out, especially in the light that attentional modulation by activity gain would be easy to implement and has been widely observed in sensory processing independent of attention.

\subsubsection{Temporal dynamics of attention}

Since one focus of this work is on the time course of attentional modulation, I will briefly review the key findings in this context. Since the beginning of attention research, experimental psychologists have been interested in the temporal dynamics of attention. Reaction time (RT) studies revealed that automatically oriented attention has two separable temporal processes. In these studies, a small salient stimulus is presented either at the location of an upcoming target (valid cue) or elsewhere (invalid cue). If the target appears shortly after the cue, RTs to the target are faster if the cue was valid compared to invalid. However, when the time between cue and target exceeds $\sim 300 \mathrm{~ms}$, RTs are typically faster to targets at the uncued location compared to the 
cued location. This latter process has been termed Inhibition of Return (IOR) and seems to be a mechanism encouraging the orienting of attention to novel locations or objects in the visual field. An experiment investigating the time course of spatial and feature-based attentional modulation during exogenous cueing of attention is part of this work (section 2.3).

Behavioral studies have come to two classes of estimates for the speed with which attention can be oriented. On the one hand, visual search tasks (Treisman and Gelade, 1980; Wolfe, 1994, 1998) have estimated shifts of attention to occur very rapidly, with very short dwell times ranging from 5-50 ms. Other behavioral experiments, however, report that attention is not a high-speed mechanism, but can only shift every several hundreds of milliseconds (Duncan, 1984; Theeuwes et al., 2004). Part of the reason for the variability between these estimates might be the fact that different tasks engage unequal contributions of exogenous or endogenous attentional orienting mechanisms. The few electrophysiological studies of the temporal dynamics of attentional modulation rather point toward latencies of several hundreds of milliseconds. Müller et al. (1998) have measured steady-state visual evoked potentials (SSVEP) in human observers during shifts of attention. The authors found that the SSVEP amplitude at electrode sites contralateral to the cued location increased during the first 400-500 ms after cue presentation, indicating a gradual build-up of attentional modulation, which was paralleled by a similar time course of target detection behavior. It should be noted though, that the SSVEP amplitude is extracted by applying a Fourier Transform to the data in sliding windows of several hundreds of milliseconds, which makes the interpretation of the reported latencies complicated. One study using extracellular single-unit recordings in area V4 has reported that the activity of single neurons is modulated within 150-300 ms after a cue to reorient attention (Motter, 1994); however, these effects were not quantified statistically. Finally, a recent study of attentional modulation in V1 during shifts of attention reports latencies between 140-210 ms (see also 2.2, Khayat et al., 2006). While these latencies might depend on the particular task used, importantly, the authors find that shifting attention to a new object increases neuronal responses $\sim 60 \mathrm{~ms}$ earlier than removing attention from an object that is no longer relevant. In one of the manuscripts (section 2.1), we will provide a quantitative analysis of the time course of attentional modulation in area MT.

In summary, higher-level cognitive influences can strongly influence the processing of sensory information and the resulting behavioral responses. 


\section{Chapter 2}

\section{Original articles and manuscripts}

This chapter is composed of the following articles and manuscripts:

- Busse, L., Katzner, S. \& Treue, S. Temporal dynamics of neuronal modulation during shifts of visual attention. Prepared for submission.

- Busse, L. \& Katzner, S. (2006). The time course of shifting visual attention. Journal of Neuroscience, 26(15), 3885-3886.

- Busse, L., Katzner, S. \& Treue, S. (2006). Spatial and feature-based effects of exogenous cueing on visual motion processing. Vision Research, 46(13), 2019-2027.

- Busse, L., Katzner, S. \& Treue, S. Effects of attention on perceptual tuning curves for direction of visual motion. Prepared for submission.

- Katzner, S., Busse, L. \& Treue, S. Object-based attention modulates activity of single neurons in primate visual cortex. Prepared for submission.

- Katzner, S., Busse, L. \& Treue, S. (2006). Feature-based attentional integration of color and visual motion. Journal of Vision, 6(3), 269-284.

- Katzner, S., Busse, L. \& Treue, S. Anticipation of impending signals lowers decision criterion without affecting perceptual sensitivity. Prepared for submission. 


\subsection{Temporal dynamics of neuronal modulation during shifts of visual attention}

Every second, primates make 3-5 saccadic eye movements, on average, bringing information from the most important aspects of the visual scene onto the most sensitive part of the eye, the fovea. The pre-motor theory of attention (Rizzolatti et al., 1987) proposes that each saccadic eye movement is preceded by a shift of attention to the saccade target. Hence, attentional shifts should be at least as frequent as saccadic eye movements. In fact, successful adaptive behavior requires that we constantly shift our focus of attention, modulating the representations of objects or locations in the visual scene according to their current behavioral relevance.

So far, most electrophysiological studies investigating the effects of attention on visual information processing have examined the steady-state effects of attention, using designs in which the animal attended to a single stimulus or location for up to several seconds (Treue and Maunsell, 1996; Treue and Martínez-Trujillo, 1999; Reynolds et al., 2000; Fries et al., 2001; McAdams and Maunsell, 1999). In contrast, only two studies have so far directly measured neuronal activity during dynamic shifts of attention (see also section 2.2, Khayat et al., 2006; Motter, 1994). Here, we examine the temporal dynamics of attentional modulation during cued shifts of attention by recording extracellular activity from individual neurons in the macaque middle temporal area (MT). We find that both automatic and voluntary shifts of attention modulate the activity of individual neurons, with the earliest effects having a latency of $\sim 100 \mathrm{~ms}$, which is in the typical range of latencies for saccadic eye movements. Furthermore, this study shows that the attentional modulations due to automatic orienting of attention precede those related to voluntary shifts of attention. Finally, after presentation of a cue signaling to shift attention, the behavioral performance of the animal is impaired for $\sim 300 \mathrm{~ms}$, further supporting the notion that shifts of attention cannot be accomplished instantaneously. 


\title{
Temporal Dynamics of Neuronal Modulation During Shifts of Visual Attention
}

\author{
Laura Busse ${ }^{1,2}$, Steffen Katzner ${ }^{1,2}, \&$ Stefan Treue ${ }^{1,2,3}$ \\ ${ }^{1}$ Cognitive Neuroscience Laboratory, German Primate Center, Kellnerweg 4, 37077 Göttingen, Germany \\ 2 Bernstein Center for Computational Neuroscience, Bunsenstrasse 10, 37073 Göttingen, Germany \\ 3 Department of Biology, Georg-Elias-Müller Institute for Psychology, University of Göttingen, \\ Gosslerstrasse 14, 37073 Göttingen, Germany
}

\begin{abstract}
Summary
Dynamically shifting attention between behaviorally relevant stimuli in the environment is a key condition for successful adaptive behavior. Here, we investigate the time course of neuronal activity during involuntary and voluntary shifts of visual attention in direction-selective neurons in monkey cortical area MT. Involuntary shifts of attention triggered by the abrupt onset of a cue are associated with a rapid modulation of activity which is followed, about $80 \mathrm{~ms}$ later, by modulation related to voluntary re-orienting of attention. These findings demonstrate that both automatic and voluntary shifts of attention modulate activity of single visual neurons and complement numerous behavioral studies showing a markedly different time course of the two attentional orienting mechanisms.
\end{abstract}

\section{Introduction}

Visual attention selectively modulates the sensory processing of information according to behavioral relevance $[4,12,14,26]$. Behaviorally, these effects are reflected in lower perceptual thresholds [e.g., 2] and faster reaction times (RTs) [e.g., 21] to stimuli within the attended region. In the past, most studies have investigated the effects of "sustained" attention, using paradigms in which the focus of attention constantly remained on a stimulus or a stream of stimuli for up to several seconds $[8,17,27,28]$. While the ability to attend to a stimulus for a prolonged period of time is certainly of great importance, many real life situations require a flexible adjustment of the focus of attention in order to adapt our processing capacities to the currently most relevant information.

So far, the time course of shifting attention has been addressed mainly by behavioral experiments. Estimates for the temporal dynamics of attention range from high-speed mechanisms of 5-50 ms [25, 29, 30] to much longer latencies of several hundred milliseconds $[5,24]$. Part of the reason for the variability between these estimates might be the fact 
that different tasks engage unequal contributions of exogenous or endogenous attentional orienting mechanisms. While the former mechanism has been described as stimulus-driven, involuntary and fast, the latter has been characterized as goal-driven, voluntary and relatively slow. Only recently, a small number of electrophysiological studies have started to shed light on the neural activity during shifts of attention $[9,13,18,19,20,31]$. These studies find that changes in behavioral relevance of a stimulus are reflected in the temporal dynamics of attentional modulation of visual activity. Estimates for latencies of these attentional modulations range from $\sim 95-500 \mathrm{~ms}$ after the instruction to shift attention. However, except for one study [9] using event-related potential (ERP) scalp recordings in human observers, these experiments did not directly compare effects of voluntary versus automatic shifts of attention on the modulation of visual activity.

Here, we investigated the temporal dynamics of attentional modulation during involuntary and voluntary shifts of covert attention. We recorded extracellular activity of direction-selective single units in cortical area MT while monkeys were attending to a moving stimulus. Unpredictably during the course of a trial, the monkeys could be cued to shift attention to a different stimulus in the visual field. The appearance of the cue triggered automatic and voluntary processes of attentional orienting which were both reflected in the neuronal responses. Neuronal modulation during automatic shifts of attention had a very short latency and was followed, only $\sim 80 \mathrm{~ms}$ later, by the modulations related to the endogenous orienting process. These findings provide evidence that automatic and voluntary shifts of attention can modulate the responses of single visual neurons, with the two orienting mechanisms differing markedly in their time course.

\section{Results}

Two macaque monkeys were trained to fixate on a fixation point and to release a lever as soon as the target, a coherently moving random dot pattern (RDP) presented at a cued peripheral location, briefly changed its direction of motion. The monkeys were also required to ignore changes in direction of motion in two other RDPs that were presented simultaneously at other locations ("distractors"). One of the three RDPs was always presented inside the classical receptive field $(\mathrm{RF})$ of the neuron under study, the other two outside; all RDPs moved either in the preferred or anti-preferred direction of the neuron. The experiment consisted of three conditions, each occurring with a relative frequency of 33\%: In "simple cueing" trials (Fig. 1A), the trial started with the presentation of the cue close to fixation pointing towards the position of the upcoming target. Target and distractor changes occurred randomly between 13-3700 ms after onset of coherent motion in the stimuli. In "shift cueing" trials (Fig. 1B), a second cue appeared, randomly between 150-2200 ms after onset of coherent motion, pointing to one of the distractors and thereby signaling the monkey to shift attention to a new target. From the moment of shift-cue onset, the monkey was only rewarded for responding to changes in the newly cued stimulus and had to ignore changes in the other two stimuli, including in the former target stimulus. The "stay cueing" trials (Fig. 1C) served as control condition. Here, the 
second cue was presented at the same position as the first cue, instructing the monkey to continue attending to the already attended stimulus.

\section{Behavioral performance}

Behavioral performance following the cue to shift attention provides a first signature for the time course of orienting attention. Fig. 2 shows hit rates (top row) and reaction times (RTs) (bottom row) averaged across recording sessions as a function of time between shift cue onset and target onset (left column) and stay cue onset and target onset (right column), respectively. The solid lines represent the least squares fit to the data using a local polynomial regression (loess), the dotted lines indicate the $95 \%$ confidence interval for the fit. In the shift cueing condition, hit rates were strongly reduced until $\sim 350 \mathrm{~ms}$ after shift cue onset. In addition, RTs were significantly increased for the fraction of correctly detected changes during approximately that time interval. This suggests that the processes of perceiving the cue, interpreting it and shifting attention cannot be executed instantaneously but require a significant amount of time. In the stay cueing condition, a small decrease in performance and an increase in RTs for correct responses with short cuetarget onset asynchronies is also evident. These effects might reflect automatic attraction of attention away from the relevant target RDP, triggered by the onset of the cue. Notably, the effects in the stay cueing condition are faster and much less pronounced than the corresponding effects during shift cueing trials.

\section{Dynamics of neuronal modulation}

To investigate the time course of attentional modulation during shifts of attention we aligned neuronal responses to the random onset of the shift or stay cue. For all subsequent comparisons we used those shift and stay cueing conditions, which were identical in sensory stimulation. Hence, we compared shift and stay-cueing conditions in which the shift/stay cue appeared at the same spatial location, but carried the information to either shift attention to or keep it focused on a particular stimulus, depending on which stimulus was attended before cue onset (see Fig. 1 B, C). Since, in shift cueing trials, the second cue appeared at a different location as the first cue, observers had to shift attention to the newly cued stimulus. In contrast, in stay cueing trials, the second cue was presented at the same location as the first cue and instructed the observers to maintain attention on the stimulus which they had already attended. Comparing neuronal activity between such shift and stay cueing conditions should reveal effects of spatial attention before cue-onset, but any such differences should decrease after cue-onset with activity ultimately being the same in both conditions.

The top row in Fig. 3 ( $A$, single neuron; $B$ recorded population) compares the time course of neuronal modulation when attention is shifted out of the RF with the corresponding control condition when the animals keep attending to a stimulus outside the RF, aligned to the onset of the shift and stay cue, respectively (dashed vertical line). In the shift cueing condition (red trace) the monkeys initially focus attention on the stimulus 

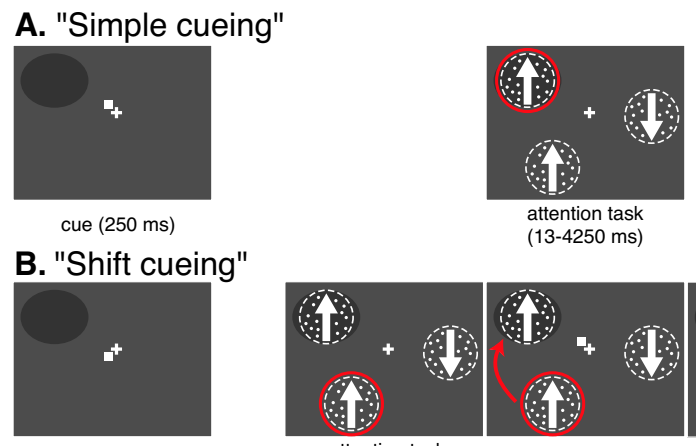

cue $(250 \mathrm{~ms})$
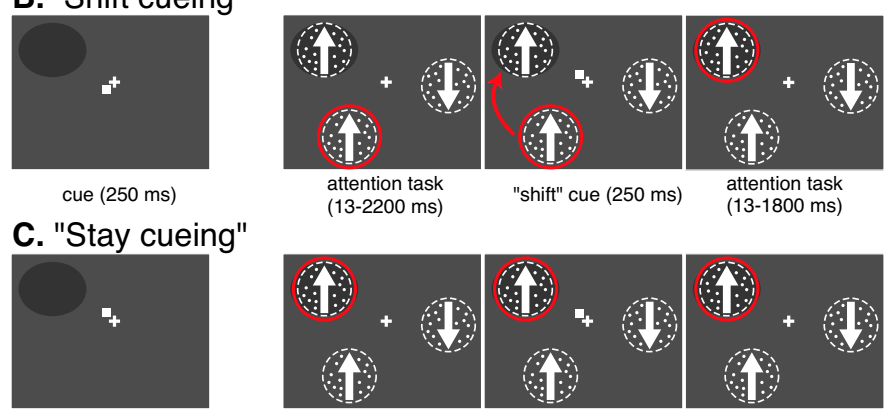

cue $(250 \mathrm{~ms})$

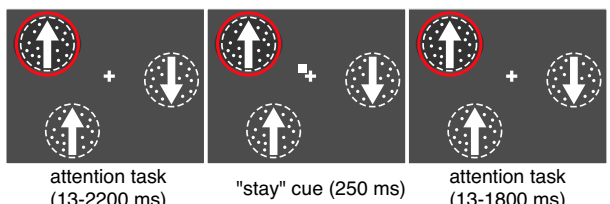

Figure 1: Schematic trial structure for the three experimental conditions. After the monkey had acquired fixation, a small white square appeared at 1.5 deg eccentricity, serving as the cue. Following a blank period of $200 \mathrm{~ms}$, three RDPs were presented at equal eccentricity, one inside the classical RF (indicated by the dark gray patch), the other two outside. The cued RDP was the "target", the other two were "distractors". The red circle indicates the focus of attention. In the "simple cueing" condition (A), the animals were rewarded for responding to brief changes in direction of motion of the target. Trials were aborted if the animals did not respond to the target change, responded to any distractor change or broke fixation. In "shift cueing" trials (B), a second cue appeared at a random time between 150-2200 ms after onset of coherent motion stimuli, instructing the observers to shift attention to the newly cued stimulus. From the moment of shift-cue onset, the observer was only rewarded for responding to direction changes in the new target. The depicted trial would be an example for the "Shift In" condition, because attention is shifted from the stimulus outside to the stimulus inside the RF. The "stay cueing" trials (C) served as control condition. Here, the second cue was presented at the same position as the first cue, signaling the observer to stay focused on the already attended stimulus. The depicted trial would be an example for the "Stay In" condition, because the cue instructs the observer to keep attending to the stimulus inside the RF. 

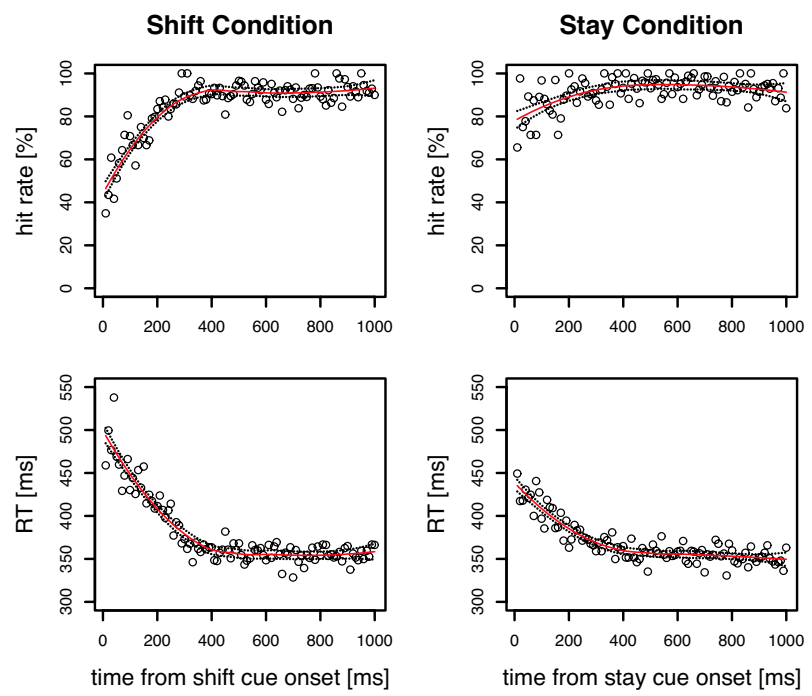

Figure 2: Behavioral signatures of shifting attention. Left column: Percentage of correctly detected targets (top) and RTs (bottom) plotted as a function of the time between shift cue- and target onset. Right column: corresponding data for the stay cueing condition. Circles represent average hit rates and average RTs across recording sessions, the solid line depicts the local polynomial regression (loess) fit to the data. Dotted lines indicate the $95 \%$ confidence interval for the fit.

inside the RF before, at time $0 \mathrm{~ms}$, the cue appears pointing towards one of the stimuli outside the RF. In contrast, in the stay cue condition (black trace), the animals attend to one of the stimuli outside the RF before and after the cue. Fig. $3 C$ (single neuron) and $D$ (recorded population) contrast the corresponding conditions in which attention is shifted from the stimulus outside the $\mathrm{RF}$ to the stimulus inside the RF (red trace) against those in which attention is cued to stay focused on the stimulus inside the RF (black trace). In all conditions, attention is only shifted across space and not across stimulus features (i.e., directions of motion), because the RDP inside the RF and the attended RDP outside the $\mathrm{RF}$ always move in the preferred direction of the neuron.

\section{Effects of attention before cue onset}

In both comparisons, the effect of spatial attention is evident before cue onset. Average activity is higher when attention is directed to the stimulus presented inside the RF compared to when it is directed to the stimulus outside the RF. To statistically evaluate this effect we calculated, for each neuron separately, an attentional index (AI) in a time window from -600 to -100 ms before cue onset: $A I=\left(f r_{A I n}-f r_{A O u t}\right) /\left(f r_{A I n}+f r_{A O u t}\right)$, where $f r_{A I n}$ and $f r_{A O u t}$ are the average firing rates if attention is directed inside and outside the $\mathrm{RF}$, respectively. Across the recorded population of neurons $(\mathrm{N}=78)$, the distribution of AI was shifted to positive values in both comparisons (Fig. 4) (Attend In vs. Attend 
A

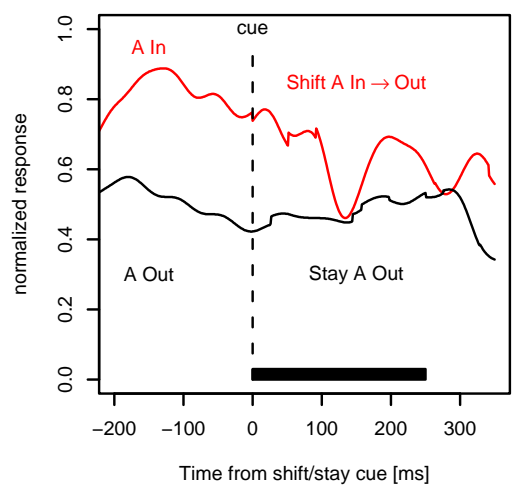

C

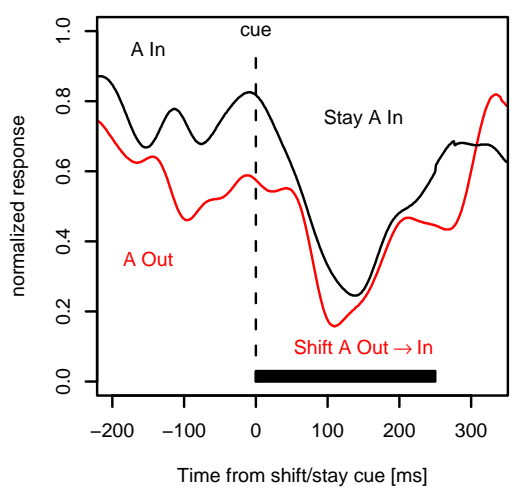

B

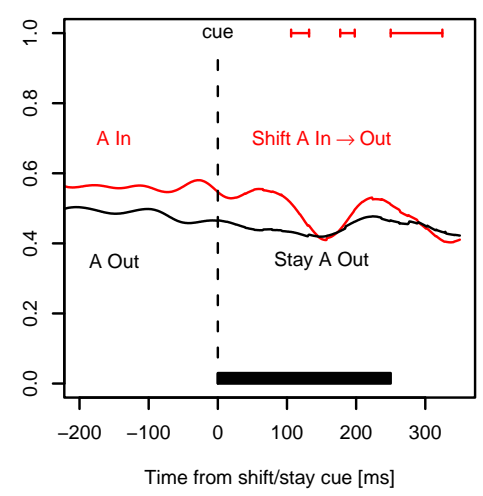

D

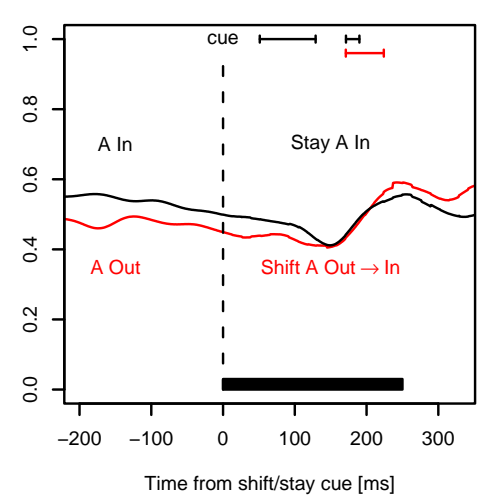

Figure 3: Time course of attentional modulation during shifts of attention. The top row shows activity of a single neuron $(A)$ and of the recorded population $(B)$ during conditions in which attention shifts away from the RF (red trace) versus stays focused on the stimulus outside the RF (black trace). The bottom row depicts activity of a single neuron (C) and of the recorded population (D) during conditions in which attention shifts from outside into the RF (red trace) versus stays focused on the stimulus inside the RF (black trace). In both cases, before the onset of the shift/stay cue (dashed line), activity is higher when attention was directed to the stimulus inside compared to outside the RF. The time course of activity in both shift and stay conditions shows components of exogenous and endogenous attentional orienting. The early decrease of activity at $\sim 120 \mathrm{~ms}$ reflects an automatic attraction of attention by the onset of the cue away from the RF because it even occurs in conditions when the cue instructed the monkey to keep attending to the stimulus inside the RF ( $\mathrm{D}$, black trace). This initial decrease is followed, $\sim 80 \mathrm{~ms}$ later, by an increase in activity. This increase can only be attributed to voluntary mechanisms of shifting attention because the cue only carried symbolic information about the target position. Interestingly, activity even increases again in conditions in which the animals were cued to shift attention out of the RF (B, red trace). This suggests that, even though the cue was perceived, the execution of the command to shift attention out of the RF has not been fully processed yet. The neuronal signature of shifting attention out of the $\mathrm{RF}$ only becomes evident at around $\sim 300 \mathrm{~ms}$. Thin horizontal red and black lines at the top of the population plots indicate $95 \%$ confidence intervals for the time spans in which changes of neuronal responses were most pronounced (see Methods). The thick black bar illustrates the duration of the cue. 
Out (before Shifting Out or Staying Out): mean, 0.06; Attend In vs. Attend Out (before Staying In vs. Shifting In): mean, 0.07). This indicates that responses are $\sim 13 \%$ and $\sim 16 \%$ higher if the stimulus inside the $\mathrm{RF}$ is attended. In both cases, the average AI is highly significantly different from 0 (one-sample $t$ test, $p<1.27^{-05}$ ).

\section{Time course of attentional modulation}

After the onset of the shift cue, activity in the Shift Out condition (Fig. 3B, red trace) decreased rapidly with the point of steepest decrease being at $123 \mathrm{~ms}$ after shift cue onset (95\% confidence interval: 106-132 ms). Across the recorded population, activity decreased in 58 out of 78 recorded neurons at this latency (mean slope $=-24.15^{-4}, p=4.49^{-5}$, one-sample $t$ test). In contrast, activity in the Stay Out control condition (black trace) did not vary considerably across time. Remarkably, although the instruction for the monkey in the Shift Out condition was to shift attention out of the RF, the initial decrease was followed by an increase that is steepest at $189 \mathrm{~ms}$ (95\% confidence interval: 177-198 ms). At this latency, activity increased in 52 out of 78 recorded neurons (mean slope $=32.42^{-4}$, $p=1.69^{-5}$, one-sample $t$ test). Only after this increase the activity again decreased (302 ms, $95 \%$ confidence interval: $250-324.7 \mathrm{~ms}$ ), with this effect being present in 48 out of 78 neurons (mean slope $=-15.39^{-4}, p=0.028$, one-sample $t$ test). Finally average activity in the Shift Out condition reached the level of activity in the Stay Out condition (time window 220-270 ms, $A I$ not significantly different from $0, p=0.09$; all following $50 \mathrm{~ms}$ intervals $p>0.164$ ).

To interpret this time course of activity, it is helpful to consider the sequence of modulations in Fig. 3D. The dynamics of activity in the Stay In condition (black trace), shortly after the onset of the stay cue, was very similar to the above described pattern. Despite the fact that, here, the appearance of the cue signalled the monkey to keep attending to the stimulus inside the RF, activity most strongly decreased at $122 \mathrm{~ms}$ after the onset of the stay cue (95\% confidence interval: $51.2-129 \mathrm{~ms}$ ). This initial decrease was evident in 54 of the 78 recorded neurons (mean slope $=-13.11^{-4}, p=0.002$, one-sample $t$ test). It was followed by an increase of activity (maximal positive slope: $181 \mathrm{~ms}, 95 \%$ confidence interval: $171.3-190 \mathrm{~ms}$ ), which was also present in the majority of recorded neurons (62 of 78 , mean slope $=22.53^{-4}, p=1.12^{-5}$ ). This increase brought activity back to approximately its level at time of cue onset. The later increase of activity in the Stay In condition is paralleled by an increase of activity in the Shift In condition (red trace), which has its point of steepest change at $193 \mathrm{~ms}$ (95\% confidence interval: 170-222 ms). Again, this increase can also be found at the level of individual neurons ( 55 of 78 , mean slope $\left.=25.19^{-4}, p=5.6^{-6}\right)$. Interestingly, the increase of activity in the Shift In condition exceeds the increase in the Stay In condition such that responses to newly attended stimuli are stronger than responses to stimuli that have been attended throughout the trial (time window 300-350 ms, $A I=0.04, p=0.017$ ).

Since activity rapidly decreases after the presentation of both the shift and stay cue, we consider this early decrease a signature of involuntary capture of attention by the sudden onset of the cue. The appearance of this stimulus might have automatically attracted 


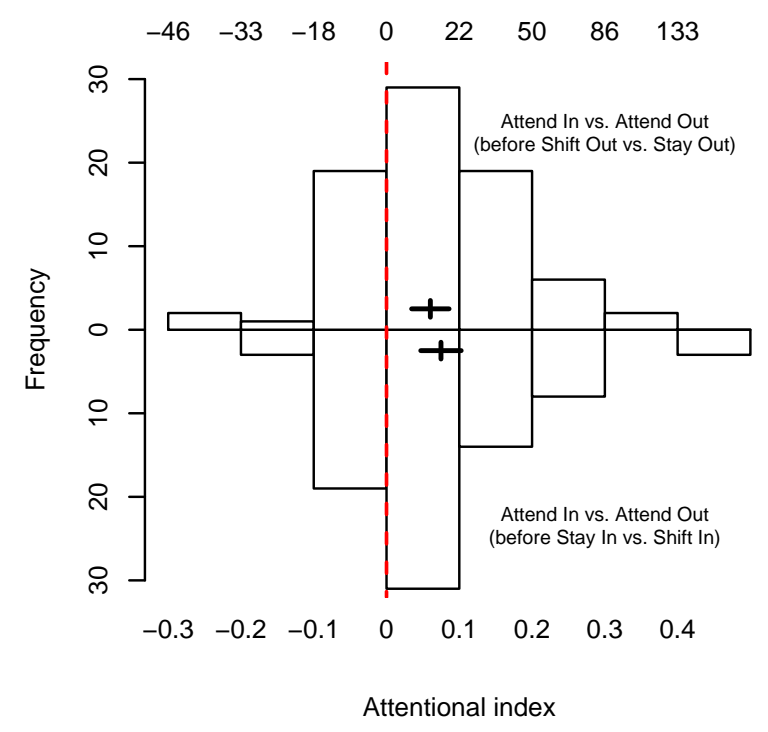

Figure 4: Distribution of the strength of attentional modulation for all recorded neurons $(\mathrm{n}=78)-600$ to $-100 \mathrm{~ms}$ before the onset of the shift or stay cue. Bottom x-axis denotes the attentional index (AI), top x-axis the corresponding modulation in percent. The top histogram shows the attention effects for conditions in which attention is directed to the stimulus inside the RF (before the instruction to Shift Out) versus outside the RF (before the instruction to maintain attention outside) (see also $3 B$ ). The bottom histogram depicts the AI for conditions in which attention is directed into the RF (before the instruction to maintain attention inside) versus outside the RF (before the instruction to Shift In) (see also $3 D$ ). Positive values indicate enhanced responses when attention is directed to the stimulus inside the RF. The cross marks the average attentional modulation, horizontal arms span the $95 \%$ confidence interval of the mean. In both conditions, the distribution of attentional modulation is shifted significantly to positive values, indicating that attention enhances responses on average by about 12.8 and $16.2 \%$, respectively. 
attention because it was behaviorally relevant for the task [see also, 10]. The later increase of activity, however, can only be due to a voluntary orienting of attention since the position of the cue close to fixation only carries symbolic information about the location of the target stimulus. Thus, the temporal dynamics of attentional modulation for the population of recorded neurons show that automatic shifts of attention triggered by the onset of the cue have a shorter latency $(\sim 70 \mathrm{~ms})$ than voluntary shifts of attention for which the position of the cue has to be interpreted.

Finally, when considering only voluntary shifts of attention, we find that shifting attention into the RF yielded an enhancement at a latency of $193 \mathrm{~ms}$, while the decrease in activity associated with an endogenous shift of attention out of the RF occurred at $302 \mathrm{~ms}$ after the cue (Fig. 3B, red trace). Thus, attentional modulation is $\sim 110$ ms shorter when attention is endogenously allocated to the stimulus inside the RF than when attention is endogenously removed from the stimulus inside the RF.

\section{Influences of the cue}

It is important to point out that the dynamics of the response modulation cannot be attributed to responses evoked by the cue. First, the cue was always presented within $1.5 \mathrm{deg}$ from fixation, i.e. far from the classical RF of most recorded neurons. Second, in all comparisons, the cue was presented at the same spatial location and thus should have equally affected activity in shift and stay conditions. Finally, the recorded population does neither respond significantly to cue onset nor cue offset (linear regression: intercept, $p=0.228$ and $p=0.67$ for on- and offset, respectively) (Fig. 5).

\section{Discussion}

This study demonstrates that switches in the behavioral relevance of a stimulus are associated with characteristic modulations of neuronal activity whose temporal dynamics depend on the nature of the orienting mechanism. Automatic capture of attention by the sudden onset of the cue could not be suppressed, even in cases when attention should be kept focused, and is reflected in a very fast decrease of neuronal activity. This decrease is followed, only $\sim 80 \mathrm{~ms}$ later, by modulations that can only be attributed to voluntary re-orienting of attention. These results are in line with numerous behavioral experiments and support the notion that endogenous and exogenous orienting are fundamentally different mechanisms of shifting attention. Furthermore, in agreement with a recent study by [13], we find that the enhancement of neuronal activity associated with the endogenous allocation of attention occurs earlier than the suppression of activity related to the endogenous removal of attention. Finally, attention seems to overcome the effect of short-term adaption in that a newly attended stimulus elicits stronger activity as compared to the same stimulus when it has been relevant before. In line with these neuronal data, we find that behavioral performance is significantly decreased for $\sim 250 \mathrm{~ms}$ after the instruction to shift attention, demonstrating that shifts of attention cannot be executed instantaneously 


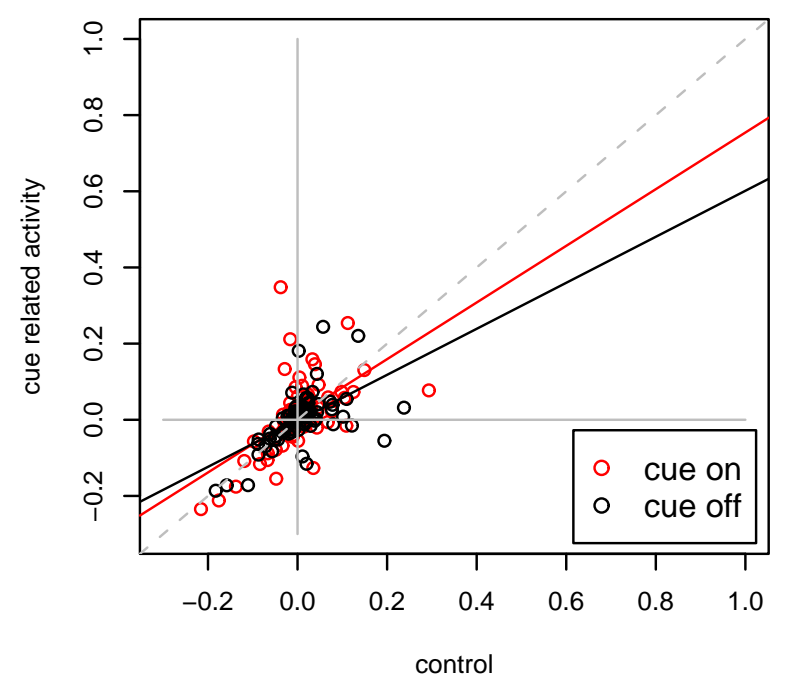

Figure 5: Cue-related sensory activity. Scatter plot of the activity evoked by cue onset and offset (ordinate) versus activity evoked in the same time windows without cue presentation (onset: red circles, offset: black circles). Activity is normalized to the peak of the response elicited by motion in the preferred direction inside the RF. On average, the recorded population does neither respond to cue onset nor offset. 
but are associated with a behavioral cost.

A large body of literature has addressed automatic and voluntary orienting mechanisms of attention but only very few studies directly compared both mechanisms. Using singleunit recordings in the lateral intraparietal area (LIP), [1] found concurrent but relatively independent effects of endogenous and exogenous attention. Whereas neurons responded strongly when a distractor was flashed inside their RF while attention was focused elsewhere in the visual field, the response of the neurons did not decrease when their receptive fields were coding the attended region and the flash occurred elsewhere. Hence, the neuronal modulation due to endogenous effects of attention did not change when attention was exogenously attracted to a different region in visual space. This is in contrast to our experiments where neuronal responses strongly decreased when attention is attracted automatically to a region outside the RF of the neuron under study. Part of the reason for this discrepancy might be the fact that the distractors used by [1] were task-irrelevant while the cue attracting attention in our study carried important information about whether to keep attending or to shift attention. This would be consistent with the view that exogenous orienting depends on top-down control [7]. One further study [9], using ERP scalp recordings in human subjects, has addressed interactions between endogenous and exogenous attention, so far. They found that exogenous attraction of attention influenced neural activity in early stages of processing, enhancing the amplitude of the P1 component between 120-150 ms latency, while endogenous attention was dominant later, modulating activity between 150-210 ms and during the P3 component (300-400 ms latency). These latencies are very well in accord with the latencies we find in the activity of single neurons.

Very recently, [13] has provided the first quantitative investigation of attentional modulations during voluntary shifts of attention using extracellular multiunit recordings in primary visual cortex (V1). Their absolute latencies (evaluated at the $50 \%$ point of a fitted curve) for shifts of attention into and out of the RF are slightly shorter than the latencies we report. The most likely explanation for this difference is that Khayat et al. [13] used a predictable point in time for the occurrence of the shift signal which could have been anticipated by the observer while the onset of our shift/stay cue was randomized across a time window of more than 2 seconds. Similar to earlier findings in V4 [18], Khayat et al. [13] report that enhancement of activity due to endogenous allocation of attention precedes the decrease of activity due to removal of attention by $\sim 60 \mathrm{~ms}$. Our data obtained from different visual area using a different paradigm are strikingly similar. We find that enhancement of activity in MT neurons associated with the allocation of attention occurs $\sim 110$ ms earlier than the decrease of activity due to voluntary orienting of attention away from the RF.

We also find that the increase of activity due to the allocation of attention exceeds the effects of short-term adaption, such that the same stimulus elicits stronger activity when it is newly attended compared to when it has been relevant throughout the duration of the trial. This effect also seems to be present in the data from Khayat et al. [13] and Motter [18], although it was not quantified there. The interaction between shortterm adaptation and attention is very well in accord with the fundamental property of the visual system to preferentially process dissimilar or novel information compared to uniform 
or constant information [11]. Intriguingly, this preference for novelty also seems to hold true for information that has recently become relevant compared to information which has been relevant for a longer period of time.

In conclusion, we show that activity in cortical area MT is modulated by attention, with the dynamics of this modulation reflecting changes in behavioral relevance on a very rapid time scale. Automatic capture of attention results in a fast modulation of activity which is followed, only $\sim 80 \mathrm{~ms}$ later by modulations that can be attributed to endogenous shifts of attention. As has been reported before in other visual areas, effects of voluntary allocation of attention precede the effects of removing attention. Finally, attention seems to overcome the effects of short-term adaptation such that newly attended stimuli elicit stronger activity than stimuli which have been relevant throughout the duration of the trial.

\section{Materials and Methods}

We recorded the responses of $\mathrm{N}=78$ direction-selective cells in area MT of two macaque monkeys to moving random dot patterns (RDPs) in conditions of sustained attention and shifts of attention. Standard surgical techniques were used [15]. Recordings were made using a one- (David Kopf Instruments) or five-channel recording system (Mini-Matrix, Thomas Recording $\mathrm{GmbH}$ ), single units were isolated using the Plexon Data Acquisition System (Plexon Inc.). Cells were determined to be from MT by their physiological characteristics (directionality and receptive field position and size) as well as by the position of the electrode in the cortex. Only responses of neurons with a direction index (ratio between responses to preferred direction and antipreferred direction) $\geq 3$ were accepted for analyses. For a given neuron, we defined as the preferred direction the peak of a Gaussian fit to the responses to 12 different directions (sampled every 30 degrees) in a condition when a single RDP was placed inside the receptive field while the animals detected a luminance change of the fixation point. The experiments in this study complied with the National Institute of Health Guide for the Care and Use of Laboratory Animals and were approved by the Regierungspräsidium Niedersachsen.

\section{Stimuli}

We used RDPs of small bright dots (density: 8 dots per $\mathrm{deg}^{2}$, luminance $117 \mathrm{~cd} / \mathrm{m}^{2}$ ) plotted within a stationary circular virtual aperture on a background of either 1 or 25 $\mathrm{cd} / \mathrm{m}^{2}$, in earlier and later recording sessions, respectively. The size of the aperture was chosen to match the boundaries of the classical receptive field $(\mathrm{RF})$ of the neuron under study as determined by a hand-mapping procedure. Movement of the dots was created by displacement of each dot by the appropriate amount at the monitor refresh rate of $76 \mathrm{~Hz}$. In every trial, we presented three RDPs of equal size, one positioned inside the recorded cell's classical RF, the other two positioned at equal eccentricity outside the cell's RF. 


\section{Behavioral task}

The monkeys were trained to attend to a moving RDP (the target) in the presence of two other moving RDPs (the distractors) while maintaining fixation on a stationary fixation cross. Each trial began with the appearance of the fixation cross. A trial started as soon as the monkey's eye position was within a fixation window of 1 deg radius centered on a (size: $0.2 \times 0.2 \mathrm{deg}$ ) fixation square. $150 \mathrm{~ms}$ after the monkey touched a lever, a white square (size: $0.35 \times 0.35 \mathrm{deg}$ ), serving as the cue, appeared at an eccentricity of $1.5 \mathrm{deg}$ at a position connecting the upcoming target RDP and the fixation point by a virtual line. The cue lasted for $250 \mathrm{~ms}$. After a blank period of $300 \mathrm{~ms}$ three RDPs appeared at different, but iso-eccentric positions on the screen. During the first $200 \mathrm{~ms}$, the RDPs were either stationary or moved in random directions ( $0 \%$ coherence). Then, RDPs coherently moved in either the preferred or anti-preferred direction of the neuron under study for the remainder of the trial. All combinations of preferred or anti-preferred directions in each stimulus were possible.

The experiment consisted of three conditions. In the "simple cueing" condition (33\% of trials), the animals obtained a liquid reward for releasing the lever in response to a direction change in the target within a response time window of $60-700 \mathrm{~ms}$ after the change. The direction change occurred randomly between 13-3700 ms after onset of coherent motion in the RDPs. The distractors could also change direction during the trial, but with a temporal separation of at least $500 \mathrm{~ms}$ from the target change. Trials in which both distractors, but not the target, changed their direction were rewarded after $4800 \mathrm{~ms}$, if the monkeys did not respond. Trials in which the monkey broke fixation or responded outside the reaction time window were considered errors and were aborted without reward. In the "shift cueing" condition (33\% of trials), the cue appeared for a second time randomly between 150-2200 $\mathrm{ms}$ after onset of coherent motion at an eccentricity of $1.5 \mathrm{deg}$ and a position connecting the fixation point with one of the other two RDPs by a virtual line (duration: $250 \mathrm{~ms}$ ). The appearance of the second cue signalled the monkey to shift attention to the newly cued RDP and, from the moment of cue onset on, to respond to direction changes in the new target. The direction changes could happen between 13-1050 ms after the second cue. Again, in case of two distractor changes the monkey was rewarded to hold the lever until trial end. In the third condition, termed "stay cueing" (33\%), the timing of events was identical to the "shift cueing" trials with the exception that the second cue re-appeared at the same position as the first cue, instructing the monkey to keep attention focused on the already attended target. The different conditions were randomly interleaved within the experiment.

\section{Data Analysis}

Data were analyzed offline using Matlab (The MathWorks, Inc.) and R [22]. Average hit rates and RTs (for correct responses only) were computed for each recording session in $10 \mathrm{~ms}$ bins (0-1000 ms) after shift and stay cue onset, respectively, before being averaged across recording sessions. Average performance was fitted using a local polynomial regres- 
sion (loess) approach, bootstrap-estimated errors of the fit were obtained by resampling residuals.

For the analysis of neuronal data, we only included correctly completed trials. We determined response rates by convolving the spike train in each trial with a Gaussian kernel (sigma $=30$ ). In shift and stay cueing conditions, we only used spikes that occurred before the first direction change (target or distractor event) after appearance of the second cue. We averaged responses across trials and normalized average responses to the peak of the transient elicited by motion in the preferred direction inside the RF, after subtraction of spontaneous firing rate. Population responses were computed by averaging across the normalized responses.

The effects of spatial attention were assessed in a time window of -600 to $-100 \mathrm{~ms}$ before onset of the second cue. For each recorded neuron, the attentional index (AI) was computed: $A I=\left(f r_{A I n}-f r_{A O u t}\right) /\left(f r_{A I n}+f r_{A O u t}\right)$, where $f r_{A I n}$ and $f r_{A O u t}$ are the mean responses if attention is directed inside and outside the RF, respectively. Only trials in which the RDP inside and the attended RDP outside the RF moved in the preferred direction of the neuron were included in the analysis. The significance of the AI was statistically evaluated using a one-sample $t$ test.

To assess the time course of neuronal modulation we determined the slope of the average activity by fitting a linear regression line to each $50 \mathrm{~ms}$ interval, shifted by $1 \mathrm{~ms}$ (from cue onset to $350 \mathrm{~ms}$ after cue onset). We then determined the time points in for which the slope was maximal and minimal, respectively. In case of the "Shift Out" condition, in which two decreases of activity were evident, we first determined the point in time of maximal slope and then the minimal slope before and after this marker. We used a bootstrapping procedure to obtain confidence intervals for the latencies of modulation. We simulated 1000 data sets by randomly selecting individual neurons from the original recorded population with replacement, for each of those we then determined the time points for maximal and minimal slopes, using the procedure described above. The distribution of obtained latencies was used to construct $95 \%$ confidence intervals based on the BCa method [6]. The slope-based method for latency estimates avoids the use of multiple statistical comparisons associated with a bin-based procedure in which the latency corresponds to the first of a number of bins that meet a significance criterion [e.g. 3, 16]. Furthermore, in comparison to procedures in which a theoretical function is fitted to the time course $[13,23]$, this procedure does not make any assumptions about the shape of the time course of activity. To evaluate latency differences between endogenous and exogenous modulations for individual neurons, we applied the slope-based procedure to Shift In and Stay In conditions and found times for maximal and minimal slopes in a window from 30 to $330 \mathrm{~ms}$ after second cue onset, respectively.

For the analysis of potential sensory activation caused by the onset of the cue, we used two conditions in which the animal directed attention to a luminance change of a square superimposed on the fixation point. We compared activity in a condition, in which the cue was irrelevant but presented at the position pointing towards the RF (onset: 50-250 ms after cue onset, offset: 50-250 ms after cue offset), to activity in the same time windows when no cue was presented. 
Dynamics of Attention

\section{References}

[1] Bisley, J. W. and Goldberg, M. E. (2003). Neuronal activity in the lateral intraparietal area and spatial attention. Science, 299(5603):81-86.

[2] Cameron, E. L., Tai, J. C., and Carrasco, M. (2002). Covert attention affects the psychometric function of contrast sensitivity. Vision Research, 42(8):949-967.

[3] Chelazzi, L., Miller, E. K., Duncan, J., and Desimone, R. (2001). Responses of neurons in macaque area V4 during memory-guided visual search. Cerebral Cortex, 11(8):761772 .

[4] Desimone, R. and Duncan, J. (1995). Neural mechanisms of selective visual attention. Annual Review of Neuroscience, 18:193-222.

[5] Duncan, J., Ward, R., and Shapiro, K. (1994). Direct measurement of attentional dwell time in human vision. Nature, 369(6478):313-315.

[6] Efron, B. and Tibshirani, R. (1993). An Introduction to the Bootstrap. Chapman and Hall, London.

[7] Folk, C. L., Remington, R. W., and Johnston, J. C. (1992). Involuntary covert orienting is contingent on attentional control settings. Journal of Experimental Psychology: Human Perception and Performance, 18(4):1030-1044.

[8] Fries, P., Reynolds, J. H., Rorie, A. E., and Desimone, R. (2001). Modulation of oscillatory neuronal synchronization by selective visual attention. Science, 291(5508):15601563 .

[9] Hopfinger, J. B. and West, V. M. (2006). Interactions between endogenous and exogenous attention on cortical visual processing. Neuroimage, Epub ahead of print.

[10] Ipata, A. E., Gee, A. L., Gottlieb, J., Bisley, J. W., and Goldberg, M. E. (2006). LIP responses to a popout stimulus are reduced if it is overtly ignored. Nature Neuroscience, 9(8):1071-1076.

[11] Kandel, E. R., Schwartz, J. H., and Jessell, T. M., editors (2000). Principles of neural science. McGraw-Hill, New York, 4th edition.

[12] Kastner, S. and Ungerleider, L. G. (2000). Mechanisms of visual attention in the human cortex. Annual Review of Neuroscience, 23:315-341.

[13] Khayat, P. S., Spekreijse, H., and Roelfsema, P. R. (2006). Attention lights up new object representations before the old ones fade away. Journal of Neuroscience, 26(1):138142.

[14] Luck, S. J., Woodman, G. F., and Vogel, E. K. (2000). Event-related potential studies of attention. Trends in Cognitive Sciences, 4(11):432-440. 
[15] Martínez-Trujillo, J. C. and Treue, S. (2004). Feature-based attention increases the selectivity of population responses in primate visual cortex. Current Biology, 14(9):744751.

[16] Maunsell, J. H. and Gibson, J. R. (1992). Visual response latencies in striate cortex of the macaque monkey. Journal of Neurophysiology, 68(4):1332-1344.

[17] Moran, J. and Desimone, R. (1985). Selective attention gates visual processing in the extrastriate cortex. Science, 229(4715):782-784.

[18] Motter, B. C. (1994). Neural correlates of feature selective memory and pop-out in extrastriate area V4. Journal of Neuroscience, 4(4):2190-2199.

[19] Müller, M. M., Teder-Sälejärvi, W., and Hillyard, S. A. (1998). The time course of cortical facilitation during cued shifts of spatial attention. Nature Neuroscience, 1(7):631-634.

[20] Murthy, A., Thompson, K., and Schall, J. (2001). Dynamic dissociation of visual selection from saccade programming in frontal eye field. Journal of Neurophysiology, 86(5):2634-2637.

[21] Posner, M. I. (1980). Orienting of attention. Quarterly Journal of Experimental Psychology, 32(1):3-25.

[22] R Development Core Team (2006). R: A Language and Environment for Statistical Computing. R Foundation for Statistical Computing, Vienna, Austria. ISBN 3-90005107-0.

[23] Roelfsema, P. R., Khayat, P. S., and Spekreijse, H. (2003). Subtask sequencing in the primary visual cortex. Proceedings of the National Academy of Sciences, 100(9):54675472.

[24] Theeuwes, J., Godijn, R., and Pratt, J. (2004). A new estimation of the duration of attentional dwell time. Psychonomic Bulletin 8 Review, 11(1):60-64.

[25] Treisman, A. M. and Gelade, G. (1980). A feature-integration theory of attention. Cognitive Psychology, 12(1):97-136.

[26] Treue, S. (2001). Neural correlates of attention in primate visual cortex. Trends in Neurosciences, 24(5):295-300.

[27] Treue, S. and Martínez-Trujillo, J. C. (1999). Feature-based attention influences motion processing gain in macaque visual cortex. Nature, 399(6736):575-579.

[28] Treue, S. and Maunsell, J. H. R. (1996). Attentional modulation of visual motion processing in cortical areas MT and MST. Nature, 382(6591):539-541. 
[29] Wolfe, J. M. (1994). Guided search 2.0: a revised model of visual search. Psychonomic Bulletin \& Review, 1:202-238.

[30] Wolfe, J. M. (1998). What can 1 million trials tell us about visual search? Psychological Science, 9(1):33-39.

[31] Woodman, G. F. and Luck, S. J. (1999). Electrophysiological measurement of rapid shifts of attention during visual search. Nature, 400(6747):867-869. 


\subsection{The time course of shifting visual attention}

This section contains a short review, published as part of the "Journal Club" feature in the Journal of Neuroscience, discussing a recent article by Khayat et al. (2006) entitled "Attention lights up new object representations before the old ones fade away". The paper is one of the first to directly measure and quantify the temporal dynamics of attentional modulation during shifts of attention. The authors recorded multi-unit activity in the primary visual cortex and demonstrate that the increase of neuronal responses due to allocation of attention occurs earlier than the decrease of activity caused by a removal of attentional resources. 
Editor's Note: These short reviews of a recent paper in the Journal, written exclusively by graduate students or postdoctoral fellows, are intended to mimic the journal clubs that exist in your own departments or institutions. For more information on the format and purpose of the Journal Club, please see http://www.jneurosci.org/misc/ifa_features.shtml.

\title{
The Time Course of Shifting Visual Attention
}

\author{
Laura Busse and Steffen Katzner \\ Cognitive Neuroscience Laboratory, German Primate Center, 37707 Göttingen, Germany \\ Review of Khayat et al. (http://www.jneurosci.org/cgi/content/full/26/1/138)
}

At any instant, we are confronted with more information than we can fully process, because our sensory and cognitive resources are limited. To cope with this avalanche we use selective attention to enhance information from the environment that we care about and suppress the rest. Extracellular single-unit recordings in the visual cortex of awake, behaving macaques have provided detailed information about the mechanisms of "sustained" attention. In these studies, attention was directed to a visual stimulus or a stream of visual stimuli for up to several seconds. Little is known, though, about "shifts" in attention. This is an important gap in our knowledge because attention must operate on millisecond time scales. Imagine driving along a busy road. While keeping your eyes on the road, you also have to attend to cars on each side, pedestrians crossing the street, upcoming signs and changing traffic lights. In such a situation, accident-free driving is only possible because we are able to quickly shift our focus of attention, thereby temporarily "lightening up" representations of the most relevant objects in the visual scene.

A recent study by Khayat et al. (2006) in The Journal of Neuroscience (http:// www.jneurosci.org/cgi/content/full/26/1/ 138) addresses how this might be achieved. The authors recorded multiunit

Received Feb. 1, 2006; accepted Feb. 17, 2006.

Correspondence should be addressed to Laura Busse, German Primate Center, Cognitive Neuroscience Laboratory, Kellnerweg 4, 37707 Göttingen, Germany. E-mail: Ibusse@gwdg.de.

D0I:10.1523/JNEUROSCI.0459-06.2006

Copyright $\odot$ 2006 Society for Neuroscience $\quad$ 0270-6474/06/263885-02\$15.00/0 activity from the primary visual cortex (V1) in two macaque monkeys while the animals performed a variant of the "curve-tracking" task, an elegant paradigm developed by Roelfsema et al. (1998) (Fig. 1A). On each trial, the monkey was presented with two curved lines, only one of which was connected via a small segment to the fixation point [Khayat et al., 2006, their Fig. 1a (http://www.jneurosci.org/cgi/content/full/26/1/138/FIG1)]. The monkey's task was to maintain its gaze on the fixation point for $800 \mathrm{~ms}$ and then saccade to the end of the target curve, the line connected to the fixation point. Roelfsema et al. (1998) have shown previously that neurons in $\mathrm{V} 1$ respond more strongly to such task-relevant curves, compared with distractor curves (i.e., curves that are task-irrelevant because they are not connected to the fixation point) [see also Khayat et al., 2006, their Fig. 2a-d (http://www.jneurosci.org/cgi/ content/full/26/1/138/FIG2)]. This selective modulation has been interpreted as a neuronal correlate of visual attention being directed to the target curve.

Khayat et al. (2006) extended the curve-tracking task to investigate the dynamics of attention. In addition to the "normal trials" described above, they introduced "switch trials," in which the monkey had to shift attention during the course of a trial (Fig. $1 B$ ), because the connection to the fixation point was changed while the monkey was waiting to saccade. Hence, the distractor curve became a target, and vice versa, thus changing the destination of the upcoming sac- cade [Khayat et al., 2006, their Fig. $1 b$ (http://www.jneurosci.org/cgi/content/ full/26/1/138/FIG1)]. The authors report that activity of neurons in area $\mathrm{V} 1$ reflects this change of behavioral relevance on a rapid time scale [Khayat et al., 2006, their Fig. $2 e-g$ (http://www.jneurosci.org/cgi/ content/full/26/1/138/FIG2)]. Remarkably, enhancement of responses caused by shifting attention to the new target curve occurred $\sim 60$ ms earlier than suppression of responses caused by removal of attention from the new distractor [Khayat et al., 2006, their Fig. 3 (http://www.jneurosci. org/cgi/content/full/26/1/138/FIG3)]. The $60 \mathrm{~ms}$ transition period, in which attention is already allocated to a new object before it is disengaged from a previously attended one, is incompatible with serial models of attentional shifts in which attention first has to be disengaged from an object before it can be shifted and allocated to another object (Shulman et al., 1979). Although Khayat et al. (2006) provide conclusive evidence against such serial models, further experiments are needed to test other models of attention that might account for the observed temporal pattern, such as split foci (McMains and Somers, 2004) or widening of the attentional focus (Müller et al., 2003).

To allow a direct comparison of neural responses during switch and normal trials the putative signal to switch attention had to occur at a fixed time $(400 \mathrm{~ms}$ in this case) after the trial begin, making the onset of the switch signal predictable. Hence, after $400 \mathrm{~ms}$, the animals were left without doubt about the task demands in the on- 
going trial ("switch" or "stay focused"). Primates have a precise internal representation of trial timing (Janssen and Shadlen, 2005), and the data of Khayat et al. (2006) suggest that their animals made use of it. In particular, responses during normal trials, in which no switch occurred, seem to decrease strongly after $\sim 550 \mathrm{~ms}$ [Khayat et al., 2006, their Fig. 3a, dotted lines (http://www.jneurosci.org/cgi/ content/full/26/1/138/FIG3)], suggesting that the monkeys' level of attention dropped after they had acquired some certainty that they were performing a normal trial. Because the authors compute the latency of attentional modulation using the difference of activity between switch and normal trials, the anticipation of the switch time might affect their estimates of absolute latencies. However, these absolute latencies do not alter their main finding, namely the relative temporal relationship between attentional enhancement and suppression.

It is remarkable that the size of the attentional enhancement after shifting attention to the new target curve seems to exceed the effect of short-term adaptation during normal trials [Khayat et al., 2006, their Fig. $3 a$, red solid line vs black dotted line (http://www.jneurosci.org/cgi/content/full/26/1/138/FIG3)]. However, because activity drops during normal trials, the extra attentional benefit for new targets is difficult to interpret. Here, it will be interesting to investigate, in further experiments, whether this additional enhancement also holds true in designs avoiding temporal predictability. This, in turn, would raise intriguing questions about the interaction between short-term adaptation and attention.

In summary, Khayat et al. (2006) provide the first detailed analyses of the temporal dynamics of attentional modulation during shifts of attention in visual area V1. Their data convincingly demonstrate that shifts of attention cannot simply be attributed to serial subprocesses that are executed sequentially but that attention can be allocated quickly to a new object even before it is disengaged from the previously attended one.

\section{References}

Janssen P, Shadlen M (2005) A representation of the hazard rate of elapsed time in macaque area LIP. Nat Neurosci 8:234-241.

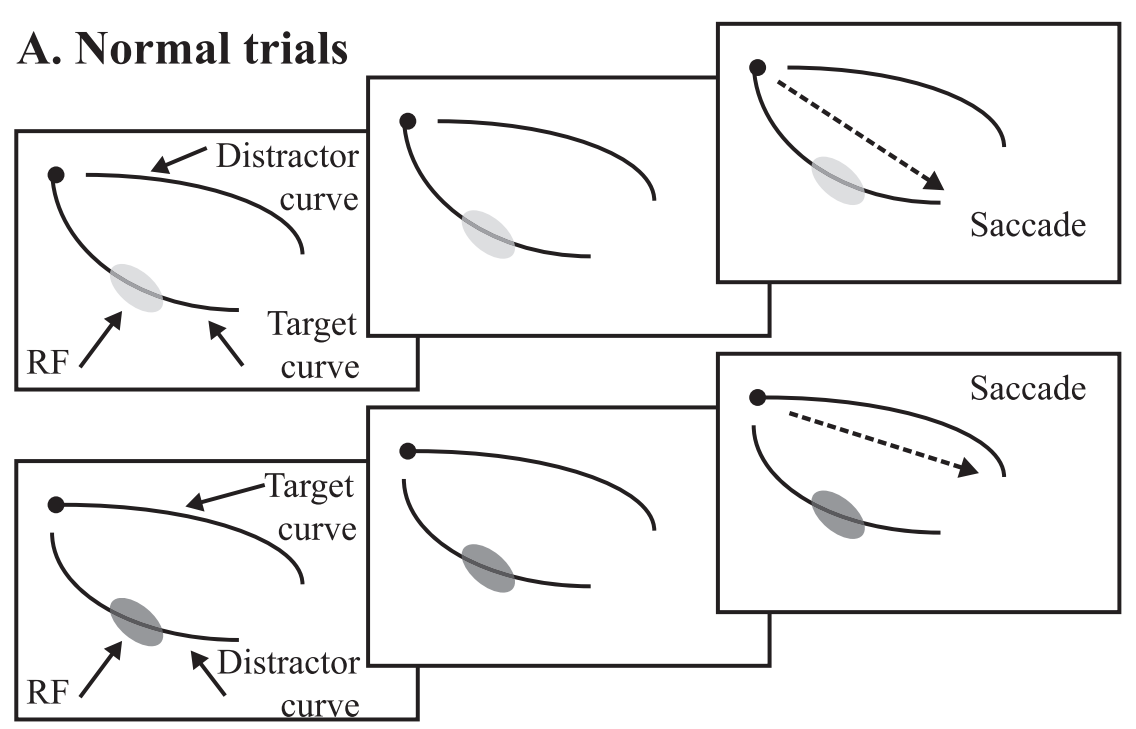

\section{B. Shift trials}

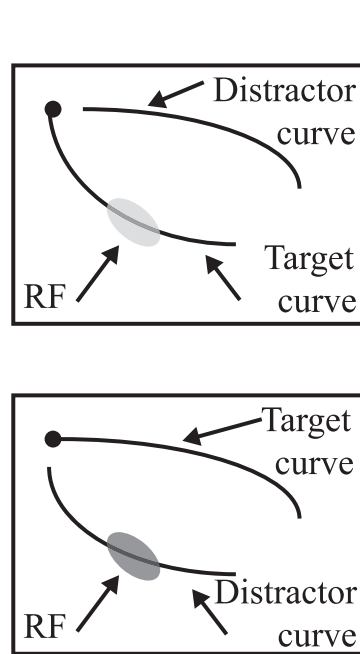

Stimulus onset Shift Cue $(\mathrm{p}=25 \%)$

$0 \mathrm{~ms}$

$400 \mathrm{~ms}$
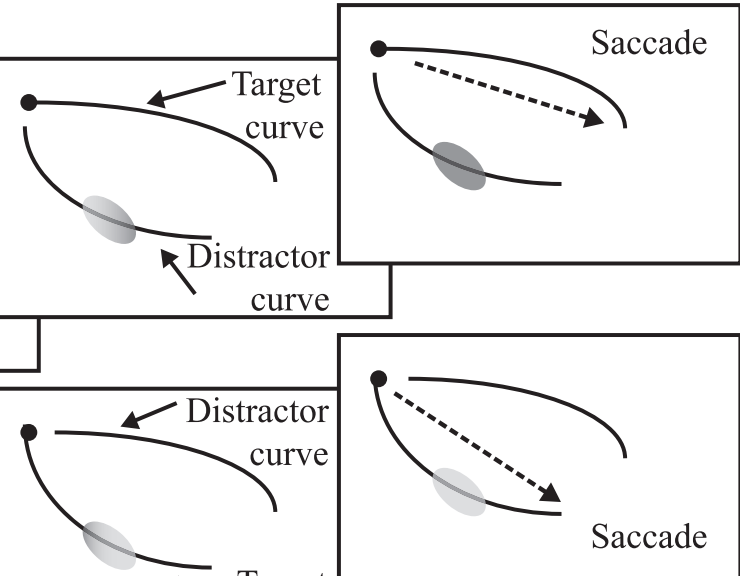

Saccade

Figure 1. Curve-tracing task in normal ( $\boldsymbol{A}$ ) and shift trials $(\boldsymbol{B})$. The shaded ellipse illustrates a V1 receptive field (RF). $\boldsymbol{A}$, During normal trials, the monkey fixates on a fixation point (black circle) for $800 \mathrm{~ms}$ before making a saccade to the end of the line connected to the fixation point (target curve). Roelfsema et al. (1998) have demonstrated an increase in V1 activity if the curve segment inside the RF belongs to the target versus distractor curve (indicated by light gray and dark gray shading, respectively). $\boldsymbol{B}$, During shift trials, the small line segment connecting the target curve to the fixation point is switched, thereby interchanging target and distractor curves. Khayat et al. (2006) show that this change of behavioral relevance is reflected in the activity of V1 neurons, leading to a fast and strong enhancement, only later followed by suppression (indicated by the gradients in the shading).

Khayat PS, Spekreijse H, Roelfsema PR (2006) Attention lights up new object representations before the old ones fade away. J Neurosci 26:138-142.

McMains SA, Somers DC (2004) Multiple spotlights of attentional selection in human visual cortex. Neuron 42:677-686.

Müller NG, Bartelt OA, Donner TH, Villringer A, Brandt SA (2003) A physiological correlate of the "Zoom Lens" of visual attention. J Neurosci 23:3561-3565.

Roelfsema PR, Lamme VA, Spekreijse H (1998) Object-based attention in the primary visual cortex of the macaque monkey. Nature 395:376-381.

Shulman GL, Remington RW, McLean JP (1979) Moving attention through visual space. J Exp Psychol Hum Percept Perform 5:522-526. 


\subsection{Spatial and feature-based effects of exogenous cueing on visual motion processing}

While the time course of the neuronal modulation during shifts of attention has not been investigated until recently, the behavioral signatures of orienting attention were among the first questions addressed in the field of attention. One very influential paradigm in this area is the so-called "Posner Cueing Paradigm" (Posner, 1980; Posner and Cohen, 1984), in which subjects are asked to make speeded responses to the appearance of a target stimulus. The target stimulus is usually preceded by a cue which can validly or invalidly indicate the spatial position of the upcoming target stimulus. Posner (1980) has first demonstrated that reaction times (RTs) to the target are faster if the preceding cue validly compared to invalidly signalled the target position. This RT benefit has been interpreted as reflecting the allocation of attention, enhancing the processing of information at the cued location.

Attention can be oriented in two fundamentally different ways. First, subjects can voluntarily, or endogenously, shift attention in response to symbolic cues, e.g. arrows presented at a distant location and pointing towards the target. Second, salient stimuli can automatically, or exogenously, attract attention to their location. Importantly, in the case of exogenous shifts of attention, the RT benefit for validly cued targets can be found only with short time intervals between cue and target. In contrast, if the time between cue and target exceeds $\sim 300 \mathrm{~ms}$, this benefit diminuishes and finally reverses, such that RTs to targets at the uncued location are faster than RTs to validly cued targets (Posner, 1980; Klein, 2000). This effect has been termed Inhibition of Return (IOR).

Here, we investigated the behavioral signatures of exogenous shifts of attention in a visual motion task. We find that the early facilitation of RTs is feature specific and occurs only for targets at the cued location moving in the cued direction. In contrast, the later inhibitory effect is purely spatial, generating an RT benefit for both the cued and uncued direction of motion at the uncued location. 


\title{
Spatial and feature-based effects of exogenous cueing on visual motion processing
}

\author{
Laura Busse $^{\text {a }}$, Steffen Katzner a, Stefan Treue ${ }^{\text {a,b, }, ~}$ \\ ${ }^{a}$ Cognitive Neuroscience Laboratory, German Primate Center, Kellnerweg 4, 37077 Göttingen, Germany \\ ${ }^{\mathrm{b}}$ Department of Biology, Georg-Elias-Müller Institute for Psychology, Göttingen University, Goßlerstrasse 14, 37073 Göttingen, Germany
}

Received 19 December 2003; received in revised form 29 April 2005

\begin{abstract}
In two experiments, we investigated the effects of exogenous cueing on visual motion processing. The first experiment shows that the typical pattern of reaction time (RT) effects, namely early facilitation and later inhibition of return (IOR), can be obtained using a color change as exogenous cue and a direction change as target. In the second experiment, we manipulated the validity of the cue independently with respect to location and feature using transparent motion stimuli. Facilitation of RTs with short cue-target interstimulus-intervals (ISIs) was only evident for targets with both the valid location and the valid feature. Furthermore, at longer cue-target intervals, RTs were prolonged for targets at the cued location, irrespective of the cued feature. These results demonstrate spatial and feature-based components of early facilitation and purely spatial IOR.
\end{abstract}

(C) 2006 Elsevier Ltd. All rights reserved.

Keywords: Visual attention; Reaction time; Motion transparency; Human

\section{Introduction}

Visual attention is the mechanism that allows us to choose behaviorally relevant information from the immense amount of input that impinges on our eyes. The flexible allocation of attention to spatial locations (e.g., Posner, 1980), different sensory features (like one particular color or motion direction $)^{1}$ (e.g., Sàenz et al., 2003), and objects (e.g., Blaser, Pylyshyn, \& Holcombe, 2000) is the central ability of our visual system to dynamically react to changing aspects of our environment and to varying behavioral goals.

In vision, cueing studies have provided insight in the dynamics of location-based, feature-based, and object-based attentional shifts. In general, such studies can be distin-

\footnotetext{
* Corresponding author. Fax: +49 5513851452.

E-mail address: treue@gwdg.de (S. Treue).

1 'Feature' refers to a particular property within a stimulus dimension, e.g., upwards motion is a feature within the stimulus dimension of motion, and red is a feature within the stimulus dimension of color (cf. Sàenz, Burac̊as, \& Boynton, 2003).
}

guished based on the nature of the cue: symbolic cues (arrows, etc.) are used for endogenous (or voluntary) orienting while peripheral cues (flashing stimuli, etc.) activate exogenous (or automatic) orienting processes (Posner \& Cohen, 1984). In the case of exogenous cueing, subjects generally react faster and more accurately after valid cues, but only for short intervals between the cue and target. If the cue-target interstimulus interval (ISI) exceeds around $300 \mathrm{~ms}$, reaction times will be slower (Posner \& Cohen, 1984), and responses less accurate (Handy, Jha, \& Mangun, 1999) for targets at the cued location than for targets at the uncued location. This latter effect of a peripheral cue has been termed Inhibition of Return (IOR) (see Klein, 2000 for a recent review). It has been suggested that IOR plays an important role in visual foraging behavior in that IOR prevents attention from permanently focussing onto or revisiting the most salient stimulus (Klein, 1988; Itti \& Koch, 2000).

The effects of exogenous cueing have not only been investigated in the spatial domain, but also with respect to feature-based and object-based attentional processes. Tipper, Driver, and Weaver $(1991,1994)$ were first to show 
object-based IOR. To dissociate location-based and objectbased IOR, they cued a moving object and found that, at long cue-target ISIs, responses were not inhibited for the initially cued location but inhibition moved with the cued object to its new location. Furthermore, with stationary stimuli, object-based and location-based inhibitory effects have been shown to combine in an additive fashion (Leek, Reppa, \& Tipper, 2003). However, other groups have failed to find independent location-based and object-based mechanisms of IOR (Christ, McCrae, \& Abrams, 2002; McAuliffe, Pratt, \& O'Donnell, 2001).

To investigate feature-based effects of IOR, typically a nonspatial stimulus attribute, such as color (Kwak \& Egeth, 1992; Law, Pratt, \& Abrams, 1995) or shape (Riggio, Patteri, \& Umilta, 2004), is repeated (valid condition) vs. non-repeated (invalid condition) for cue and target. The results obtained in these studies are mixed, in that some groups found feature-based inhibitory effects (Law et al., 1995; Riggio et al., 2004), whereas others did not (Kwak \& Egeth, 1992). However, in most of the studies, spatial and nonspatial attributes of the stimuli have not been manipulated independently (e.g., cues and targets were always presented at fixation (Kwak \& Egeth, 1992; Law et al., 1995)), thereby confounding spatial and feature-based effects of IOR. In addition, repetition of stimulus features might lead to adaptation (at least when presented at the same location) (e.g., Clifford, 2002; Muller, Metha, Krauskopf, \& Lennie, 1999) or repetition blindness (Fox \& de Fockert, 2001; Kanwisher, 1987; Taylor \& Klein, 1998).

Here, we investigate spatial and feature-based effects of exogenous cueing on visual motion processing using an experimental design that allows the independent manipulation of spatial and nonspatial stimulus attributes. In the first experiment, we show that spatial IOR can be obtained using a cue-target combination of stimulus attributes that are processed in two different visual pathways (Felleman \& Van Essen, 1991; Ungerleider \& Mishkin, 1982), namely a color change (ventral pathway) serving as the cue and a change in motion direction (dorsal pathway) serving as the target. In the second experiment, we independently vary the validity of the cue with respect to location and feature in order to disentangle spatial and feature-based effects of exogenous cueing. With short cue-target ISIs, we find facilitation of RTs only when the cue is valid with respect to both location and feature. Additionally, in conditions with longer cue-target ISIs, we obtain IOR for the cued location, irrespective of the previously cued feature. These results demonstrate location- and feature-based components of exogenous shifts of attention.

\section{Methods}

\subsection{Experiment 1}

Ten naive subjects (age 20-28, 6 female, 4 male) with normal or corrected-to-normal vision participated in the experiment. All subjects gave informed written consent and were paid for taking part in an one hour session in which they completed 5 blocks of 100 trials each. One subject was excluded from the data analysis since his performance was more than three standard deviations below the sample mean.

The experiment was conducted in a dimly illuminated and quiet room. Stimuli were presented on a VGA monitor (Quatographic, Color Station Professional) operated at a refresh rate of $85 \mathrm{~Hz}$ and a spatial resolution of 40 pixels/deg. Stimulus presentation and recording of responses was controlled by custom-made software running on an Apple Power Mac G4. Subjects placed their head on a chin-rest or a bite-bar positioned $57 \mathrm{~cm}$ from the monitor. During the trials, eye-movements were monitored using an infrared eyetracking system (ISCAN ETL-200). In case eye position deviated more than $1.5 \mathrm{deg}$ from a central fixation point the experiment was paused by the experimenter and subjects were re-instructed to maintain fixation.

The stimulus was composed of two circular apertures (radius $1.8 \mathrm{deg}$ ) of moving dots (dot density: 8 pixels $/ \mathrm{deg}^{2}$ ) centered $5 \mathrm{deg}$ to the left and the right of the fixation point. Dots were gray $\left(12 \mathrm{~cd} / \mathrm{m}^{2}\right)$ on a black background, subtending $0.05 \mathrm{deg}$ of visual angle in width. The dots in each aperture coherently moved at a speed of $7 \mathrm{deg} / \mathrm{s}$, in one of 4 possible directions $(45,135,225$, and $315 \mathrm{deg}$ deviation from vertical). In each trial, the directions of the two dot patterns differed by at least $90 \mathrm{deg}$. During the course of the trial, some dots changed their color to red. For each participant separately, flicker fusion photometry was conducted to achieve sub-

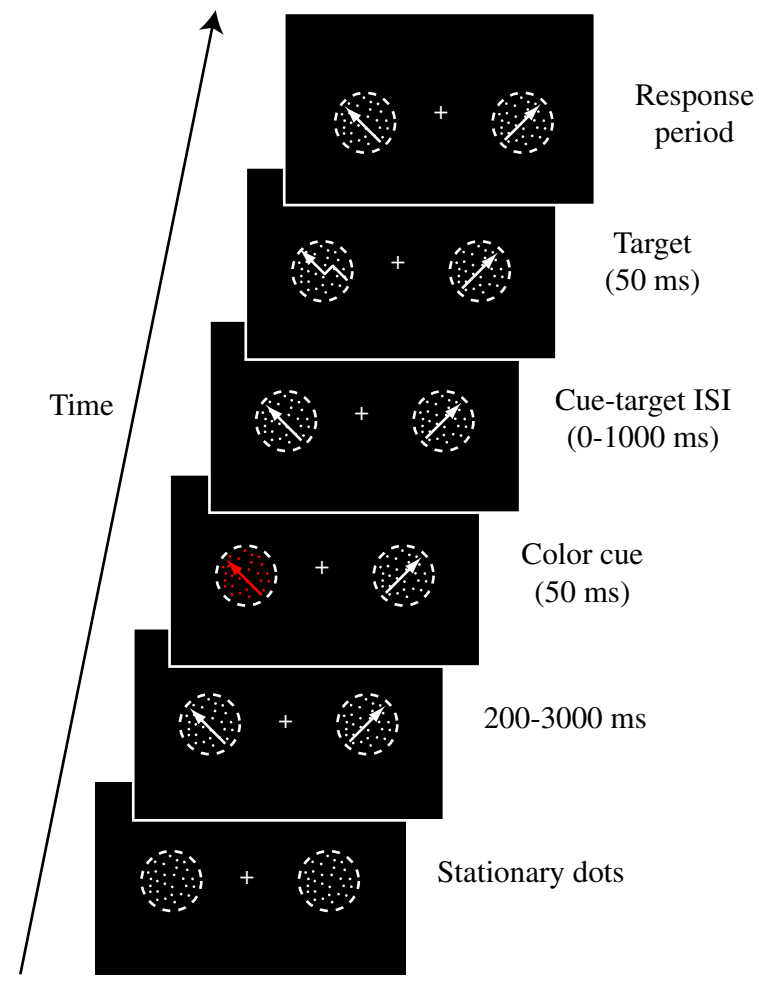

Fig. 1. Schematic trial structure for Experiment 1. Two random dot patterns were presented to the left and right of fixation. After trial start, the dots in the apertures moved coherently in directions differing by at least 90 deg for $200-3000 \mathrm{~ms}$. Randomly during this time period a cue consisting of a color change to red ( $50 \mathrm{~ms}$ duration) appeared in one of the apertures. This cue was followed by a target defined as a brief change in the direction of motion (except for a 'catch trial' condition, in which no target was presented, see below). The subjects' task was to respond as quickly as possible to the direction change (or to withhold the response in case of a 'catch trial'). The example illustrates a valid trial since the target direction change occurs in the previously cued motion pattern. Note that the dashed outlines of the apertures as well as the arrows symbolizing the movement of the dots were not present in the actual experiment. (For interpretation of the references to color in this figure legend, the reader is referred to the web version of this paper.) 
jective isoluminance for the gray and red colors. While the intensity of the gray was held constant for all subjects, the intensity of the red gun was adjusted by each subject until minimal flicker was perceived at a flicker rate of $16 \mathrm{~Hz}$. The adjusted intensity of the red gun was averaged across 25 trials and the result was used in Experiment 1.

Fig. 1A shows a schematic layout of the trial structure. At the beginning of each trial, stationary dots were presented in the apertures. When subjects initiated the trial by pressing the space bar on a computer keyboard, the dots in the two apertures started moving.

Seventy-five percent of the trials were 'cued trials,' $25 \%$ were 'catch trials' (Fig. 2). For the 'cued trials,' three different conditions were presented with equal probabilities, namely valid, invalid, and neutral trials. Hence, across trials cues were completely unpredictive with respect to the upcoming target location. In the valid and invalid trials, 200$3000 \mathrm{~ms}$ after trial start, the dots in one of the apertures changed their color to red. The color change lasted for $50 \mathrm{~ms}$ and served as the exogenous cue. A variable time interval $(0-1000 \mathrm{~ms})$ after cue-offset (cue-target ISI), the dots in either the same (valid condition) or the opposite (invalid condition) aperture underwent a direction change of $23 \mathrm{deg}$ and returned to their original direction after another $50 \mathrm{~ms}$. In the neutral trials, the color change occurred in both stimuli such that no particular location was cued. For all 'cued trials' the subjects' task was to detect the direction change and respond with a keypress ('H') as quickly as possible. Reaction times below $100 \mathrm{~ms}$ were considered anticipatory responses, reaction times above $1000 \mathrm{~ms}$ were counted as misses. The 'catch trials' were identical to the 'cued trials' except that no direction change (i.e., target) followed the cue, i.e., the dots continued to move in their original direction until trial end (signal absent trials). Thus, no response was required. 'Catch trials' were randomly interleaved with the 'cued trials' and served the purpose of preventing subjects from forming temporal expectancies and making anticipatory responses. After each trial, subjects received auditory feedback.

For each condition, RTs for correct 'cued trials' were sorted according to the cue-target ISI into 5 bins of $200 \mathrm{~ms}$ width (0-200, 200-400, 400-600, $600-800$, and $800-1000 \mathrm{~ms}$ ). For the statistical analysis of reaction times, a two-way ANOVA with the within-subject factors cueing (valid, invalid, neutral) and ISI (5 levels) was used. When appropriate, significance levels were corrected for violations of the sphericity assumption using the Greenhouse-Geyser method; however, original degrees of freedoms are reported.

\subsection{Experiment 2}

In Experiment 2, the same circular apertures and trial sequence as in Experiment 1 were used (Fig. 3). Here, each stimulus consisted of two superimposed populations of dots moving in opposite directions, resulting in a percept of transparent motion. The two dot populations always moved in the same opposite directions in the two apertures (45/225 deg or $135 / 315$ deg deviation from vertical). Stimulus parameters were identical to those used in Experiment 1 except for dot density. In Experiment 2, each individual surface contained only half the dots $\left(4\right.$ pixels $\left./ \mathrm{deg}^{2}\right)$ in order to keep the overall dot-density constant across experiments. To compensate for the reduced dot density in each single surface the duration of the cue color change was doubled to $100 \mathrm{~ms}$.

Using two transparent motion stimuli allowed the presentation of two different features (i.e., motion directions) superimposed at a single spatial location, and to repeat the same feature (i.e., same motion direction) at two different spatial locations. Since the color-cue and the directiontarget always occurred in only a single motion direction in a single dot pattern, we were able to independently manipulate the cue-validity with respect to location and feature (Fig. 4). Again, the cue was entirely unpredictive regarding the location and the direction of motion of the subsequent target.

For example, in the 'valid location, valid direction' condition the target occurred in the same location and in the same motion direction as the preceding cue. Analogously, the 'invalid location, valid direction' condition consisted of a target direction change occurring in the dot pattern opposite from the cued dot pattern, but in the population of dots moving in the same motion direction as the population of dots that served as the cue. The combinations of cue validity with respect to location (valid/invalid location) and motion direction (valid/invalid feature) yielded four different trial types. Additionally, a 'neutral condition' was presented in which the cue appeared simultaneously in one surface of each stimulus, such that no particular location or direction was cued. As in Experiment 1, the ratio of 'cued trials' and 'catch trials' was 3:1, cued surface, location and the directions of motion were randomized across trials.

Twelve naive subjects (age: 20-31, 5 female, 7 male) participated in two $1 \mathrm{~h}$ sessions conducted at different days. One subject was excluded from the data analysis because performance was below $50 \%$ in the catch trial condition ( $44 \%$ correct). As in the first experiment, trials were sorted into bins of $200 \mathrm{~ms}$ width according to the cue-target ISI. For the statistical
A

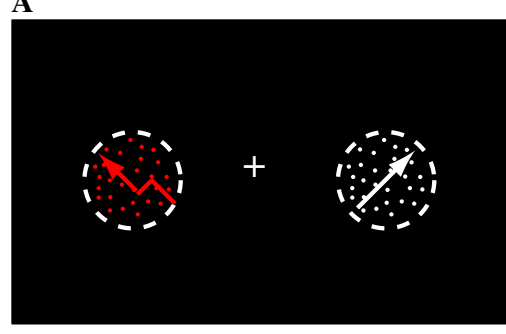

C

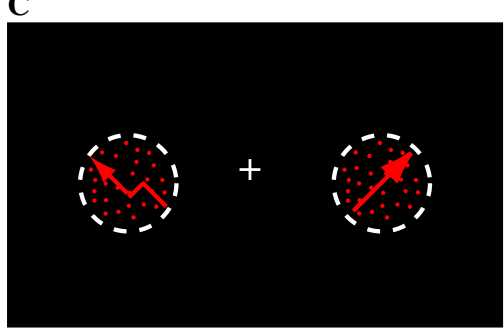

B

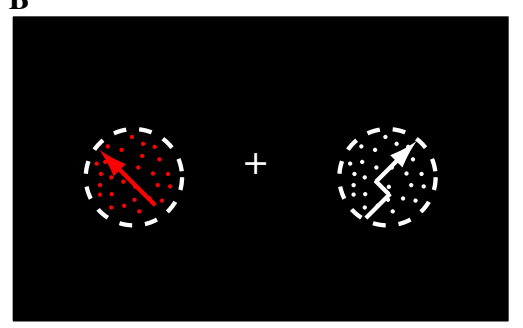

D

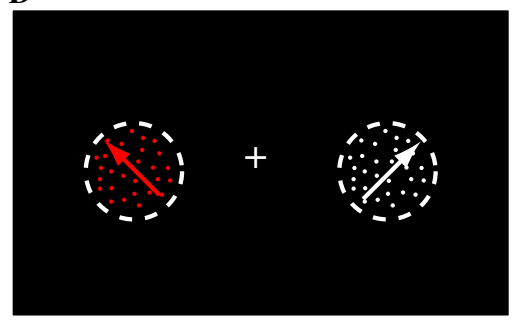

Fig. 2. Conditions for Experiment 1. For purpose of illustration the cue is drawn in the same frame as the target, which was not the case in the actual experiment (see Fig. 1). (A) Valid condition: cue and target in the same aperture. (B) Invalid condition: cue and target in different apertures. (C) Neutral condition: the cue appears in both apertures. (D) Catch trial: no target. The ratio of catch trials was $25 \%$, conditions (A-C) were presented with equal probabilities. The location of the cue and the two directions of motion were randomly varied across trials. 


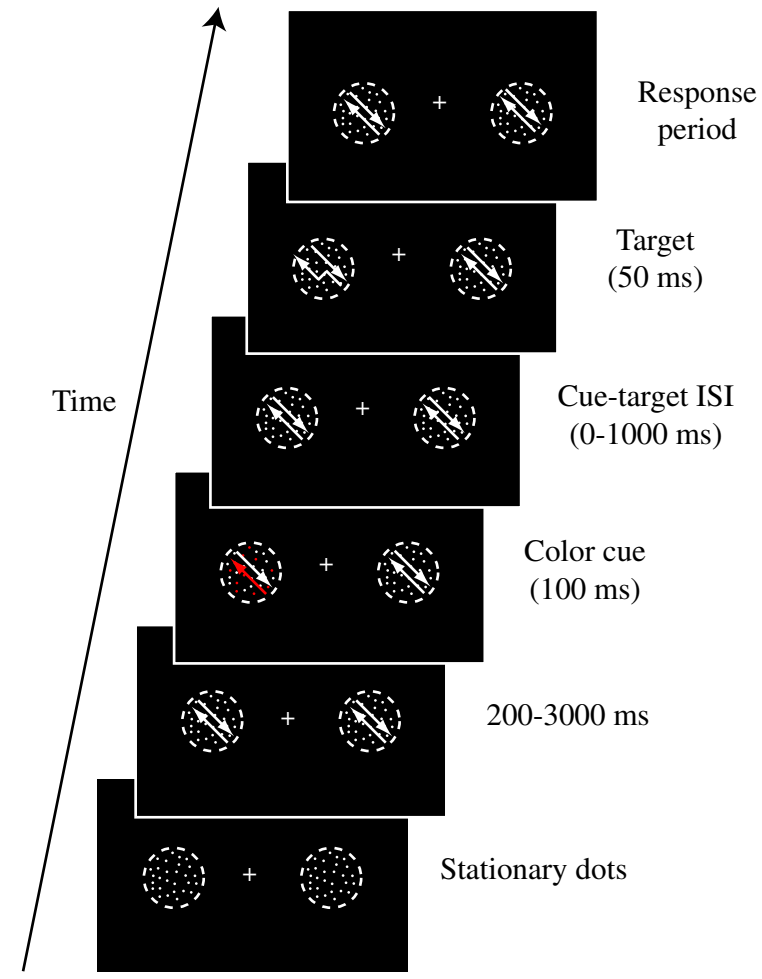

Fig. 3. Schematic trial sequence for Experiment 2. Two superimposed surfaces moving in opposite directions were presented to the left and right of fixation. After $200-3000 \mathrm{~ms}$ a color change in one surface served as the exogenous cue. Since the cue appeared only in a single direction of motion at a particular location we can independently manipulate the validity of the cue with respect to location and feature. The subjects' task was to respond as quickly as possible to the change in direction that could follow within $1000 \mathrm{~ms}$ after cue offset. In neutral trials, the cue was presented in one direction of each aperture, such that no particular location or feature was cued. In 'catch trials' no target followed the cue. The example illustrates a trial in the condition 'valid location, valid direction' since the target occurs in the cued aperture and in the cued direction of motion.

analysis of RT data, a three-way ANOVA with the within-subject factors location (valid, invalid), direction (valid, invalid), and ISI (5 levels) was used. When appropriate, significance levels were corrected using the Greenhouse-Geyser correction; however, original degrees of freedom are reported. RTs in the neutral condition were compared against RTs in the other cued trials in post-hoc comparisons.

\section{Results}

\subsection{Experiment 1}

In the first experiment, we investigated whether an exogenous color-cue affects processing of subsequent motion targets. Across subjects, performance for cued trials was $94.8 \%$, for catch trials $96.5 \%$.

Mean reaction times for Experiment 1 are shown in Fig. 5. The ANOVA for RTs revealed a significant main effect of ISI $(F(4,32) \mathrm{D} 8.35, p<0.01)$, indicating that RTs vary as a function of cue-target ISI. More importantly, the effects of exogenous cueing depend on the level of cue-target ISI, as reflected in the significant interaction between these factors $(F(8,64) \mathrm{D} 2.56, p<0.05)$. In trials with short cue-target ISIs (0-200 ms), subjects were faster in both the valid and neutral condition than in the invalid condition (462/470 ms vs. $496 \mathrm{~ms}$, respectively; $p<0.05$, NewmanKeuls). In contrast, for longer cue-target ISIs (200-400 and $400-600 \mathrm{~ms}$ ), this pattern first disappears and then reverses. This crossover effect is significant for ISIs between 400 and $600 \mathrm{~ms}$ with responses to targets after valid and neutral cues being on average $13 \mathrm{~ms}$ slower than responses to targets at uncued locations (436/433 ms vs. $422 \mathrm{~ms}$, respectively; $p<0.05$, Newman-Keuls). For longer cue-target ISIs, there was no significant difference between the conditions.

\subsection{Experiment 2}

In Experiment 2, we investigated location- and featurebased effects of exogenous cueing, using transparent motion stimuli. In this experiment, performance for cued trials $(76.9 \%)$ and catch trials $(82.1 \%)$ were lower compared to Experiment $1(t(18) \mathrm{D} 4.9, p<0.001$ for cued trials, and $t(18) \mathrm{D} 2.4, p<0.05$ for catch trials), and mean RTs across all conditions were slower (444 ms vs. $507 \mathrm{~ms}$; $t$ (18) D 2.2, $p<0.05)$ in Experiment 2 than Experiment 1. These differences probably reflect an increased target detection difficulty due to the presence of a second, overlapping dot surface.

Mean RTs for Experiment 2 are shown in Fig. 6. Along with the increased RTs compared to Experiment 1 goes an increase of the crossover latency in which facilitation changes to inhibition (600-800 ms bin). This agrees with the proposal that the onset of IOR might vary with the difficulty of the task (Lupiáñez, Milan, Tornay, Madrid, \& Tudela, 1997; Lupiáñez, Milliken, Solano, Weaver, \& Tipper, 2001). The reasoning is as follows: the more difficult the task, the more attention will be devoted to the target processing, and hence to the processing of the cue (Folk, Remington, \& Johnston, 1992); the more intensely attention will be allocated to the cue, the longer attention will dwell on it (Klein, 2000), and the longer facilitation will last after the exogenous cue.

An overall three-way ANOVA (location $f$ direction $f$ ISI) on RTs revealed a main effect of ISI $(F(4,40)$ D 5.96, $p<0.05)$, indicating that RTs vary as a function of cue-target ISI. A main effect of location $(F(1,10) \mathrm{D} 5.25, p<0.05)$ indicates that, on average, responses to targets at the cued location were faster than responses to targets at the uncued location ( $491 \mathrm{~ms}$ vs. $507 \mathrm{~ms}$ ). This effect is, on average, larger for targets with the cued feature as revealed by the interaction between location and direction $(F(1,10)$ D 5.69, $p<0.05)$. In addition, we obtain a significant interaction between location and ISI $(F(4,40) D 7.32, p<0.001)$, showing that the effect of spatial cueing changes, on average, with ISI. Most importantly, the three-way interaction is also significant (location $\mathrm{f}$ direction $\mathrm{f}$ ISI, $F(4,40)$ D 3.03, $p \mathrm{D} 0.05)$. Post hoc comparisons revealed significant differences between the cueing conditions for the ISI bins 0-200, 200-400, and 600-800 ms. For the first and second ISI bin, 
A

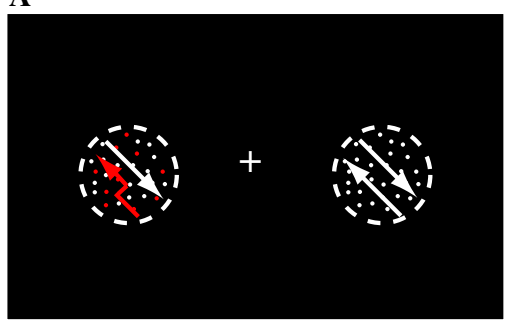

C

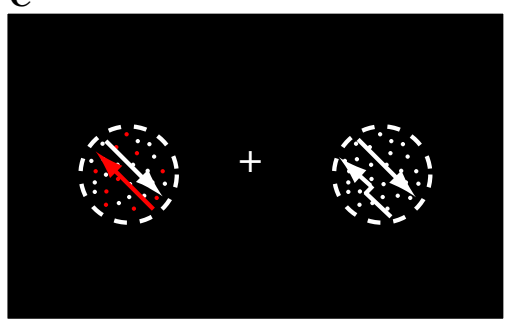

E

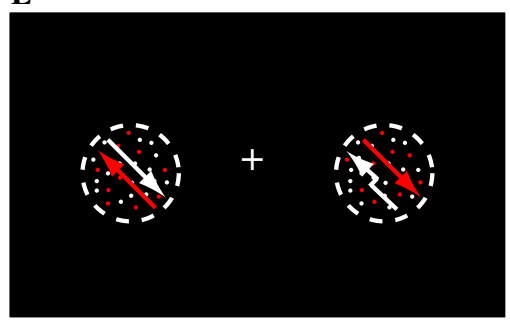

B

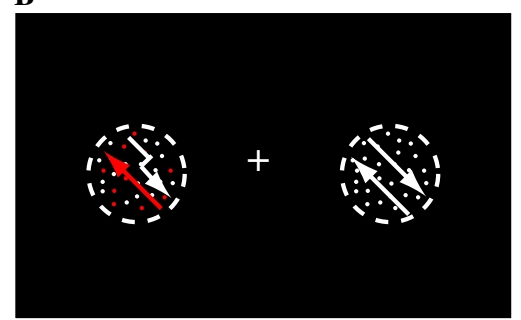

D

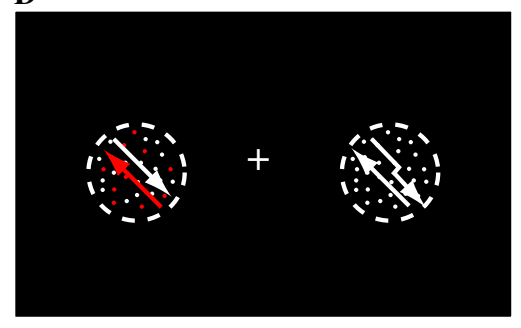

$\mathbf{F}$

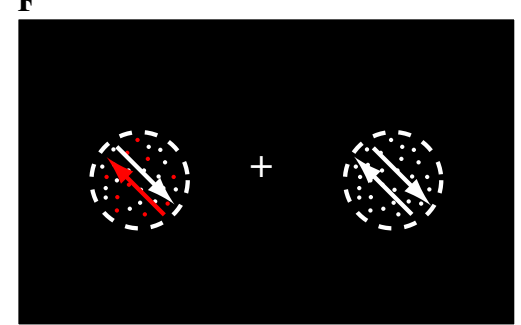

Fig. 4. Conditions in Experiment 2. (A) Valid location, valid direction. (B) Valid location, invalid direction. (C) Invalid location, valid direction. (D) Invalid location, invalid direction. (E) Neutral condition. (F) Catch trial. The ratio of catch trials was $25 \%$. Conditions (A-E) were presented with equal probability. Note that, in the actual experiment, the cue and the target were not presented at the same time.

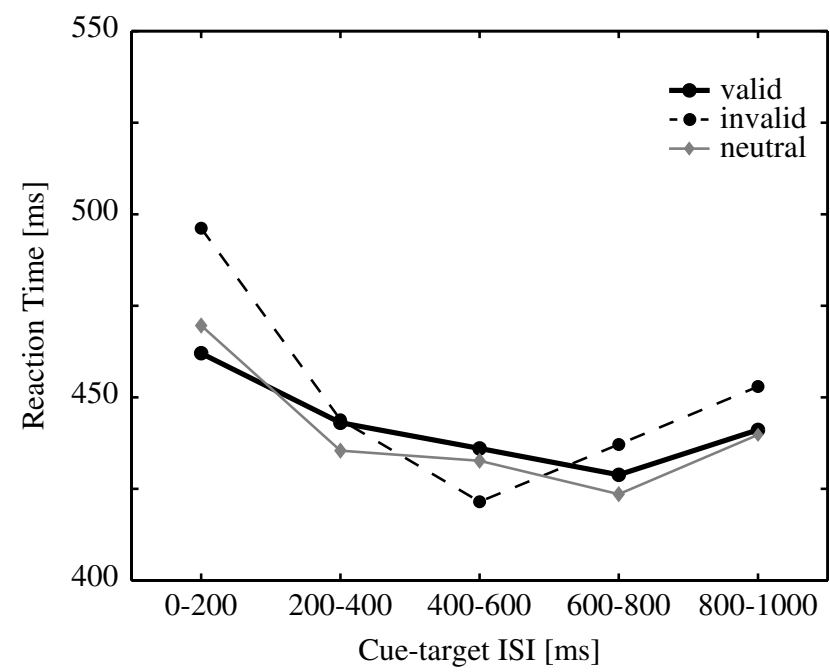

Fig. 5. Mean reaction times for Experiment 1. In conditions with short cue-target ISIs RTs are faster after both valid and neutral cues compared to invalid cues (466 ms vs. $496 \mathrm{~ms}$; 0-200 ms ISI). However, for longer cuetarget ISIs, this pattern reverses, and targets in the uncued dot pattern are responded to faster compared to both cued and neutral targets $(435 \mathrm{~ms}$ vs. $422 \mathrm{~ms}$; 400-600 ms ISI).

a separate two-way ANOVA with factors location and direction revealed a main effect of location $(F(1,10)$ D 10.77, $p<0.01$ (0-200 ms ISI); $F(1,10)$ D 6.42, $p<0.05$
(200-400 ms ISI)), indicating that, on average, responses to targets in the cued location were faster than responses to targets in the uncued location. Moreover, we obtain a significant interaction between location $€$ feature $(F(1,10) \mathrm{D} 8.82, p<0.05$ (0-200 ms ISI), $F(1,10) \mathrm{D} 11.28$, $p<0.01(200-400 \mathrm{~ms}$ ISI)). Post hoc comparisons show that RTs are fastest to targets appearing at the cued location and in the cued feature ('valid location, valid direction'), while there is no significant difference between the other cueing conditions $(496 \mathrm{~ms}$ vs. $565 \mathrm{~ms}$ for $0-200 \mathrm{~ms}$, $483 \mathrm{~ms}$ vs. $522 \mathrm{~ms}$ for $200-400 \mathrm{~ms} ; p<0.05$, NewmanKeuls). Additionally, there is a significant benefit for targets at the cued location and with the cued feature compared to the neutral condition for the ISI bin $0-200 \mathrm{~ms}$ ( $496 \mathrm{~ms}$ vs. $538 \mathrm{~ms} ; p<0.05$, Newman-Keuls). This difference vanishes in the subsequent ISI bin. A cross-over of RTs is evident in the $600-800 \mathrm{~ms}$ bin. Here, RTs to targets at the cued location are slower than RTs to targets at the uncued location $(483 \mathrm{~ms}$ vs. $456 \mathrm{~ms}, \quad F(1,10) \mathrm{D} 5.52$, $p<0.05$, main effect of location). Importantly, this effect does not depend on the cued feature $(F(1,10) \mathrm{D} 0.16$, $p \mathrm{D} 0.6$, interaction between feature and location). There is also no significant difference between RTs to targets at the cued location and RTs in the neutral condition for both the third (400-600 ms) and fourth ISI (600-800 ms) periods. 


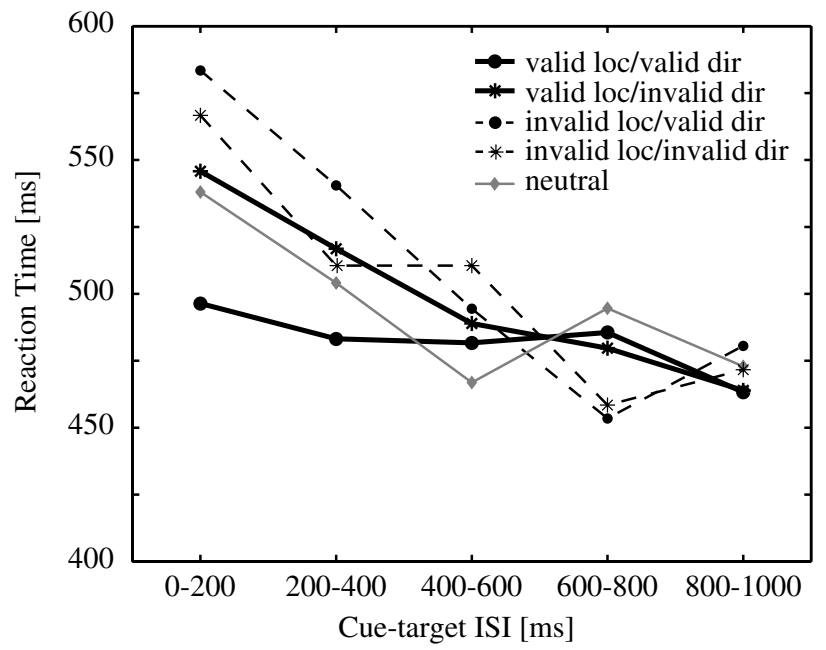

Fig. 6. Mean reaction times for experiment 2. At cue-target ISIs between 0-200 ms and 200-400 ms RTs are fastest if the target occurs in the previously cued location and direction ( $496 \mathrm{~ms}$ vs. $565 \mathrm{~ms}$ for $0-200 \mathrm{~ms} ; 483 \mathrm{~ms}$ vs. $522 \mathrm{~ms}$ for $200-400 \mathrm{~ms}$ ) compared to the other cueing conditions. While for the earliest targets, a benefit of the 'valid location, valid direction' compared to the neutral condition $(538 \mathrm{~ms})$ is present, this difference disappears between 200 and $400 \mathrm{~ms}$. At longer cue-target intervals (600$800 \mathrm{~ms}$ ), RTs are slower for targets appearing in the cued location compared to the uncued location ( $483 \mathrm{~ms}$ vs. $456 \mathrm{~ms}$ ). There is no difference between RTs in the neutral condition and RTs to validly cued targets in neither the third or fourth ISI period.

\section{Discussion}

In two experiments, we show spatial and feature-based effects of exogenous cueing on the processing of visual motion using an experimental design that does not confound cueing of location and cueing of feature. We find that, with short cue-target ISIs, detection of a change in direction of motion at the cued location is facilitated as evidenced by faster RTs to validly cued targets (Experiment 1). When further dissociating spatial and feature-based effects of the cue (Experiment 2), it becomes evident that this facilitation is feature-specific, such that targets occurring in the cued aperture (location) and in the cued direction of motion (feature) yield the fastest responses. In contrast, when the cue-target ISI is in the range of 400 $600 \mathrm{~ms}$ (Experiment 1) or 600-800 ms (Experiment 2), RTs to targets at the cued location are prolonged. This late inhibitory effect on RTs seems to be purely spatial.

By using a cue-target combination consisting of two different stimulus attributes processed in different visual pathways, namely a color change serving as a cue and a direction change constituting the target, we can infer that simple sensory interactions are not likely to cause the sequence of facilitatory and inhibitory effects of the cue. Furthermore, since we are not repeating the same feature for the cue and the target, we can exclude repetition blindness as an explanation for the effects in our experiments (Fox \& de Fockert, 2001). Rather, we interpret the findings as attention being initially reflexively drawn to the cued feature at the cued location, and later being oriented away from such tagged locations.

\subsection{Mechanisms and neuronal substrates}

Although the effects of exogenous cueing have been studied extensively using behavioral techniques, the underlying physiological processes remain unclear. So far, few studies have investigated the brain areas and mechanisms involved in automatic orienting as well as the physiological consequences of exogenous cueing on sensory processing.

\subsubsection{Initial attraction of attention}

In line with numerous previous studies, we find an initial facilitation of RTs to cued compared to uncued targets for short cue-target ISIs in both experiments. In Experiment 1, this initial difference does not seem to be due to a speed-up of RTs relative to the neutral condition (although the data show a trend in this direction), but seems caused primarily by prolonged responses to targets at the uncued location. In contrast, in the second experiment, RTs in the "valid location, valid feature' condition are considerably faster than the neutral and all other cueing conditions. This difference between the two experiments is surprising, and we can only speculate about the causes: Maybe processing of the neutral cue led to fast responses in the first experiment since task difficulty was low and attention was attracted in a split-focus fashion (e.g., Awh \& Pashler, 2000; McMains \& Somers, 2004) to both apertures with little or no cost after the neutral cue. Further experiments testing different neutral cues are needed to examine the facilitatory component after exogenous cueing relative to a neutral condition.

A number of recent studies employed transparent motion stimuli to investigate the effects of exogenous cueing on motion processing. They found that, after a salient change in one of two spatially superimposed, rotating surfaces performance in a subsequent discrimination task was strongly reduced for the uncued surface (e.g., Mitchell, Stoner, Fallah, \& Reynolds, 2003; Reynolds, Alborzian, \& Stoner, 2003; Valdés-Sosa, Cobo, \& Pinilla, 2000). This benefit in performance for discrimination in the cued surface lasted for a couple of hundreds of milliseconds. Thus, the facilitatory effect on performance has a similar time-course than the RT effect we observed in Experiment 2. The fact that the three studies cited above did not vary spatial location but presented the cue and target at fixation might have disguised a potential subsequent locationbased inhibition that would have affected both features similarly.

As has been proposed in computational models of bottom-up attentional processes (Itti \& Koch, 2000; Itti \& Koch, 2001), location-based facilitation of RTs with short cue-target ISIs could be interpreted as arising from a peak in activity in a feature-unspecific, retinotopically organized global 'salience map,' reflexively drawing attention to the most salient (i.e., cued) location. This 'salience map' is thought to receive input from multiple feature-specific 
maps that each encode spatial contrast in one specific feature dimension, like orientation, color, or motion. Directing attention to the location corresponding to the peak activity in such a feature-unspecific salience map would yield a purely spatial-based attentional advantage. Evidence for representation of salience, not stimulus features per se, has been found for neurons in the pulvinar (Robinson \& Petersen, 1992), parietal cortex (Gottlieb, Kusunoki, \& Goldberg, 1998), in the frontal eye fields (FEF) (Thompson \& Schall, 2000; Thompson, 2001), and in the superior colliculus (SC) (Kustov \& Robinson, 1996). However, to account for a feature-based component in the initial orienting of attention as found in Experiment 2 one would need to assume an additional, feature-specific mechanism, e.g., incorporation of feature information in the global saliency map or interactions with distributed feature-specific computations of salience (e.g., Desimone \& Duncan, 1995; Hamker, 2004).

\subsubsection{Inhibition of return}

IOR has been proposed to be a crucial mechanism of attentional orienting in that it prevents attention from permanently focusing on the most salient stimulus (Itti \& Koch, 2001; Klein, 1988). According to this notion, attention is able to shift to different stimuli with decreasing saliency in the visual scene by transient inhibition of neurons in the 'salience map' encoding the attended stimulus (Itti \& Koch, 2000, 2001).

Experiments 1 and 2 both demonstrated IOR for longer cue-target ISIs when targets appeared at the cued location. Consistently across the experiments, this disadvantage for the previously cued location seems to be primarily caused by a benefit for the uncued location, since RTs to targets in the uncued location are considerably faster than RTs to targets after both spatially valid and neutral cues. Thus, instead of being a true inhibition of the attended stimulus, the effect might rather be interpreted as a facilitation of previously unattended locations. On the other hand, our neutral cue, consisting of a salient change in both apertures, might have led to similar inhibitory processes than the valid cue. To disentangle these opposing interpretations further experiments using a different neutral cue, e.g., a change at fixation, need to be conducted.

The finding that IOR seems to operate in a purely spatial manner has immediate functional plausibility. ${ }^{2}$ When searching for an item with defining characteristics (e.g., a certain color) in a cluttered visual scene spatial IOR biases the system toward processing of new locations if the item has not been found at the previously attended location. This mechanism seems crucial for efficient attentional employment. In contrast, it would seem an inappropriate strategy if the attended feature, which defines the target item, would be inhibited across the entire visual field by a global feature-based IOR.

\footnotetext{
${ }^{2}$ We thank one anonymous reviewer for pointing out this issue.
}

Single-unit recordings in the lateral intraparietal (LIP) area in awake behaving monkeys have revealed a potential neural correlate of a transient spatial-based inhibition (Bisley \& Goldberg, 2003). In this study, typical effects of exogenous cueing on behavior (lower contrast thresholds with short ISIs, higher contrast thresholds with long ISIs for targets at the cued location) correlate with the ensemble activity in LIP. Shortly after a transient cue-related activity, the LIP population response is increased for neurons with receptive fields (RF) responding to the cued region compared to neurons whose RFs are located at the opposite target location. After a period of $80-90 \mathrm{~ms}$ without any significant difference between the two population responses, the firing rate of the neurons coding the cued region decreases below the firing rate of those coding the opposite target location.

Along the lines of the motor theory of IOR (Rafal, Calabresi, Brennan, \& Sciolto, 1989; Sapir, Soroker, \& Berger, 1999), single unit studies in the superior colliculus (SC), a critical node in the visual orienting pathway, propose a collicular contribution to IOR (Dorris, Klein, Everling, \& Munoz, 2002; Fecteau, Bell, \& Munoz, 2004). For short cue-target intervals, Fecteau et al. (2004) showed that the initial facilitation of saccadic RTs is accompanied by an enhanced neural response to the target in the SC. Similarly, prolonged RTs correlate with a suppression of the target-related responses in the SC (Dorris et al., 2002; Fecteau et al., 2004) and reduced target-related responses at the cued location have been found in the event-related scalp potential (ERP) recorded in humans. At long cue-target intervals, P1 amplitudes are reduced when targets appear at the cued location, suggesting that exogenous cueing can influence sensory processing in the extrastriate cortex (Hopfinger \& Mangun, 1998; McDonald, Ward, \& Kiehl, 1999). Using event-related fMRI IOR has been linked to areas commonly activated during oculomotor/motor planning (frontal and supplementary eye fields) (Lepsien \& Pollmann, 2002; Mayer, Seidenberg, Dorflinger, \& Rao, 2004) as well as attentional orienting (posterior parietal, superior and middle temporal, anterior cingulate and thalamic areas) (Mayer et al., 2004).

\subsection{Perceptual effects vs. shifts of criterion}

Traditionally, the effects of stimulus-driven or bottomup capture of attention have been interpreted as reflecting perceptual effects. Recently, an alternative explanation has been advanced, namely a simple change in criterion for targets at attended vs. unattended locations (Eckstein, Thomas, Palmer, \& Shimozaki, 2000; Verghese, 2001). In case of the Posner cueing paradigm, Eckstein, Shimozaki, and Abbey (2002) propose an ideal-observer model that weights the information at cued and uncued locations as a function of the cue validity. They find close agreement of psychophysical data with the model and no evidence for a change in the quality of the visual signal, which suggests a 
change in criterion rather than a perceptual benefit of attention. While RT data cannot distinguish between shifts of sensitivity vs. criterion, one should note that simple weighting of information according to the cue-validity cannot account for effects after uninformative precues as used in this study. Using a signal-detection measure of perceptual sensitivity in a design with unpredictive cues, Handy et al. (1999) showed that the typical pattern of RTs, namely early facilitation and later IOR, is also found for sensitivity measures like $d^{\prime}$ and $A^{\prime}$. Similarly, an irrelevant singleton in a search array seems to attract attention such that target detectability seems reduced (Theeuwes, Kramer, \& Kingstone, 2004). Finally, Carrasco, Ling, and Read (2004) recently demonstrated that uninformative exogenous cues alter the perceived contrast of the cued items, at least with a short cue-target ISI. Unfortunately, our design does not allow us to determine the signal detection measures $d^{\prime}$ and $\beta$ in order to directly test for changes in sensitivity vs. decision criterion. While it is straightforward to calculate hit rates for the various cue-target ISIs for the different signal present conditions, it is impossible (except for the neutral cueing condition) to categorize false alarms with respect to the various valid or invalid cueing conditions. Moreover, there is no obvious way to sort the false alarms into the cue-target ISI bins. Clearly, further experiments will be needed to dissociate changes in sensitivity from changes in criterion over time in an exogenous cueing task.

\section{Summary}

In summary, we show spatial and feature-based influences of exogenous cueing on motion processing. By using a cue-target combination that avoids simple sensory interactions we infer that exogenous automatic attentional processes can best account for the results. With short cuetarget ISIs, we find a benefit for targets at the valid location and with the valid feature, probably reflecting attentional orienting to the cued item. In contrast, with long cue-target ISIs, responses to targets are slower when they occur at the cued location irrespective of the cued feature. This finding underlines the notion that IOR might facilitate orienting to novel spatial locations.

\section{Acknowledgments}

We thank Annette Heinrich for testing some of the subjects in Experiment 2 and for providing help with the figures. This study was funded in part by support from the state of Lower Saxony.

\section{References}

Awh, E., \& Pashler, H. (2000). Evidence for split attentional foci. Journal of Experimental Psychology: Human Perception and Performance, 26(2), 834-846.

Bisley, J., \& Goldberg, M. (2003). Neuronal activity in the lateral intraparietal area and spatial attention. Science, 299(5603), 81-86.
Blaser, E., Pylyshyn, Z., \& Holcombe, A. (2000). Tracking an object through feature space. Nature, 408, 196-199.

Carrasco, M., Ling, S., \& Read, S. (2004). Attention alters appearance. Nature Neuroscience, 7(3), 308-313.

Christ, S., McCrae, C., \& Abrams, R. (2002). Inhibition of return in static and dynamic displays. Psychonomic Bulletin \& Review, 9(1), 80-85.

Clifford, C. (2002). Perceptual adaptation: Motion parallels orientation. Trends in Cognitive Sciences, 6(3), 136-143.

Desimone, R., \& Duncan, J. (1995). Neural mechanisms of selective visual attention. Annual Review of Neuroscience, 18, 193-222.

Dorris, M., Klein, R., Everling, S., \& Munoz, D. (2002). Contribution of the primate superior colliculus to inhibition of return. Journal of Cognitive Neuroscience, 14(8), 1256-1263.

Eckstein, M., Shimozaki, S., \& Abbey, C. (2002). The footprints of visual attention in the posner cueing paradigm revealed by classification images. Journal of Vision, 2(1), 25-45.

Eckstein, M. P., Thomas, J. P., Palmer, J., \& Shimozaki, S. S. (2000). A signal detection model predicts the effects of set size on visual search accuracy for feature, conjunction, triple conjunction, and disjunction displays. Perception \& Psychophysics, 62(3), 425-451.

Fecteau, J., Bell, A., \& Munoz, D. (2004). Neural correlates of the automatic and goal-driven biases in orienting spatial attention. Journal of Neurophysiology, 92(3), 1728-1737.

Felleman, D., \& Van Essen, D. (1991). Distributed hierarchical processing in the primate cerebral cortex. Cerebral Cortex, 1, 1-47.

Folk, C., Remington, R., \& Johnston, J. (1992). Involuntary covert orienting is contingent on attentional control settings. Journal of Experimental Psychology: Human Perception and Performance, 18(4), 1030-1044.

Fox, E., \& de Fockert, J. (2001). Inhibitory effects of repeating color and shape: Inhibition of return or repetition blindness? Journal of Experimental Psychology: Human Perception and Performance, 27(4), 798812.

Gottlieb, J., Kusunoki, M., \& Goldberg, M. (1998). The representation of visual salience in monkey parietal cortex. Nature, 391(6666), 481-484.

Hamker, F. H. (2004). A dynamic model of how feature cues guide spatial attention. Vision Research, 44, 501-521.

Handy, T., Jha, A., \& Mangun, G. (1999). Promoting novelty in vision: Inhibition of return modulates perceptual-level processing. Psychological Science, 10(2), 157-161.

Hopfinger, J., \& Mangun, G. (1998). Reflexive attention modulates processing of visual stimuli in human extrastriate cortex. Psychological Science, 9(6), 441-447.

Itti, L., \& Koch, C. (2000). A saliency-based search mechanism for overt and covert shifts of visual attention. Vision Research, 40(10-12), 1489-1506.

Itti, L., \& Koch, C. (2001). Computational modelling of visual attention. Nature Review Neuroscience, 2(3), 194-203.

Kanwisher, N. (1987). Repetition blindness: Type recognition without token individuation. Cognition, 27(2), 117-143.

Klein, R. (1988). Inhibitory tagging system facilitates visual search. Nature, 334(6181), 430-431.

Klein, R. (2000). Inhibition of return. Trends in Cognitive Sciences, 4, 138147.

Kustov, A., \& Robinson, D. (1996). Shared neural control of attentional shifts and eye movements. Nature, 384(6604), 74-77.

Kwak, H.-W., \& Egeth, H. (1992). Consequences of allocating attention to locations and to other attributes. Perception \& Psychophysics, 51, 455-464.

Law, M., Pratt, J., \& Abrams, R. (1995). Color-based inhibition of return. Perception \& Psychophysics, 57, 402-408.

Leek, E., Reppa, L., \& Tipper, S. (2003). Inhibition of return for objects and locations in static displays. Perception \& Psychophysics, 65(3), 388-395.

Lepsien, J., \& Pollmann, S. (2002). Covert reorienting and inhibition of return: An event-related fMRI study. Journal of Cognitive Neuroscience, 14(2), 127-144.

Lupiáñez, J., Milan, E., Tornay, F., Madrid, E., \& Tudela, P. (1997). Does IOR occur in discrimination tasks. Yes, it does, but later. Perception \& Psychophysics, 59(8), 1241-1254. 
Lupiáñez, J., Milliken, B., Solano, C., Weaver, B., \& Tipper, S. (2001). On the strategic modulation of the time course of facilitation and inhibition of return. Quarterly Journal of Experimental Psychology, 54(3), 753-773.

Mayer, A., Seidenberg, M., Dorflinger, J., \& Rao, S. (2004). An eventrelated fMRI study of exogenous orienting: Supporting evidence for the cortical basis of inhibition of return? Journal of Cognitive Neuroscience, 16(7), 1262-1271.

McAuliffe, J., Pratt, J., \& O’Donnell, C. (2001). Examining location-based and object-based components of inhibition of return in static displays. Perception \& Psychophysics, 63(6), 1072-1082.

McDonald, J., Ward, L., \& Kiehl, K. (1999). An event-related brain potential study of inhibition of return. Perception \& Psychophysics, 61(7), 1411-1423.

McMains, S., \& Somers, D. (2004). Multiple spotlights of attentional selection in human visual cortex. Neuron, 42(4), 677-686.

Mitchell, J., Stoner, G., Fallah, M., \& Reynolds, J. (2003). Attentional selection of superimposed surfaces cannot be explained by modulation of the gain of color channels. Vision Research, 43(12), 1323-1328.

Muller, J., Metha, A., Krauskopf, J., \& Lennie, P. (1999). Rapid adaptation in visual cortex to the structure of images. Science, 285(5432), 1405-1408.

Posner, M. (1980). Orienting of attention. Quarterly Journal of Experimental Psychology, 32, 3-25.

Posner, M., \& Cohen, Y. (1984). Components of visual orienting. In H. Bouma \& D. Bouwhuis (Eds.), Attention and performance $X$ (pp. 531555). Hillsdale, NJ: Erlbaum.

Rafal, R., Calabresi, P., Brennan, C., \& Sciolto, T. (1989). Saccade preparation inhibits reorienting to recently attended locations. Journal of Experimental Psychology: Human Perception and Performance, 15(4), 673-685.

Reynolds, J., Alborzian, S., \& Stoner, G. (2003). Exogenously cued attention triggers competitive selection of surfaces. Vision Research, 43(1), 59-66.

Riggio, L., Patteri, I., \& Umilta, C. (2004). Location and shape in inhibition of return. Psychological Research, 68, 41-54.
Robinson, D., \& Petersen, S. (1992). The pulvinar and visual salience. Trends in Neurosciences, 15(4), 127-132.

Sàenz, M., Burac̊as, G., \& Boynton, G. (2003). Global feature-based attention for motion and color. Vision Research, 43(6), 629-637.

Sapir, A., Soroker, N., \& Berger, A. (1999). Inhibition of return in spatial attention: Direct evidence for collicular generation. Nature Neuroscience, 2(12), 1053-1054.

Taylor, T., \& Klein, R. (1998). Inhibition of return to color: A replication and nonextension of Law, Pratt, \& Adams (1995). Perception \& Psychophysics, 60, 1452-1456.

Theeuwes, J., Kramer, A., \& Kingstone, A. (2004). Attentional capture modulates perceptual sensitivity. Psychonomic Bulletin \& Review, 11(3), $551-554$.

Thompson, K., \& Schall, J. (2000). Antecedents and correlates of visual detection and awareness in macaque prefrontal cortex. Vision Research, 40(10-12), 1523-1538.

Thompson, K. G. (2001). From attention to action in frontal cortex. In J. Braun, C. Koch, \& J. Davies (Eds.), Visual attention and cortical circuits (pp. 137-157). MA: MIT Press.

Tipper, S., Driver, J., \& Weaver, B. (1991). Object-centred inhibition of return of visual attention. Quarterly Journal of Experimental Psychology. A, Human Experimental Psychology, 43(2), 289-298.

Tipper, S., Weaver, B., Jerreat, L., \& Burak, A. (1994). Object-based and environment-based inhibition of return of visual attention. Journal of Experimental Psychology: Human Perception and Performance, 20(3), 478-499.

Ungerleider, L., \& Mishkin, M. (1982). Two cortical visual systems. In D. Ingle, M. Goodale, \& R. Mansfield (Eds.), Analysis of visual behavior (pp. 49-86). MA: MIT Press.

Valdés-Sosa, M., Cobo, A., \& Pinilla, T. (2000). Attention to object files defined by transparent motion. Journal of Experimental Psychology: Human Perception and Performance, 26(2), 488-505.

Verghese, P. (2001). Visual search and attention: a signal detection theory approach. Neuron, 31(4), 523-535. 


\subsection{Effects of attention on perceptual tuning curves for direc- tion of visual motion}

Recently, a new method, termed spike-triggered averaging, has been developed to determining the fine structure of receptive fields of single neurons (Chichilnisky, 2001). Here, the average stimulus preceding a spike is computed, yielding the best linear approximation of the neuron's spatio-temporal impulse response function (Dayan and Abbott, 2001). In the motion domain, one advancement of the method is the motion reverse-correlation technique (MRC) (Borghuis et al., 2003; Perge et al., 2005). Here, the spike train of the neuron under study is reverse correlated with a pseudo-random sequence of brief, fully coherent motion impulses in one of various directions and speeds to determine its direction and speed tuning across time.

For this study, we devised a psychophysical variant of the MRC method to obtain direction tuning curves for human observers. We asked observers to make speeded responses to a designated target direction within the motion impulse sequence and cross-correlated their behavioral responses with that sequence. First, we show that the psychophysical version of the MRC method yields significant direction tuning curves, from which, e.g., the target direction can be readily extracted. The width of these perceptual tuning curves is considerably narrower than the width of tuning curves found for single neurons in area MT, which indicates that the psychophysical MRC method does not only measure direction tuning but also assesses non-sensory, decision-related influences. Second, we have tested the influence of attention on these tuning curves. We find that, on the system's level, attention increases the amplitude and decreases the width of perceptual tuning curves for direction of motion. 


\title{
Effects of attention on perceptual tuning curves for direction of visual motion
}

\author{
Laura Busse $^{1,2}$, Steffen Katzner ${ }^{1,2}$, Christine Tillmann ${ }^{1} \&$ Stefan Treue ${ }^{1,2}$ \\ ${ }^{1}$ Cognitive Neuroscience Laboratory, German Primate Center, Kellnerweg 4, 37077 Göttingen, Germany \\ 2 Bernstein Center for Computational Neuroscience, Bunsenstrasse 10, 37073 Göttingen, Germany
}

\begin{abstract}
In sensory neurophysiology, reverse-correlation analyses have advanced our understanding of the spatio-temporal structure of receptive fields and the tuning properties of individual neurons. Here, we developed a psychophysical variant of the motion reverse correlation (MRC) technique to investigate how visual selective attention influences human perceptual tuning curves for direction of motion. Observers were required to make speeded responses to a designated target direction embedded in a random sequence of brief, fully coherent motion impulses. Reverse correlating behavioral responses with motion impulses yielded correlation functions which were most pronounced for motion directions around the target direction, occurring 600-250 ms before the behavioral response. From these correlation functions, we constructed perceptual tuning curves, which were well described by Gaussian model functions. A comparison of the perceptual tuning curves between conditions differing in attentional load revealed that attention increased the amplitude and decreased the width of the tuning curves. These results show that attention can enhance both the dynamic range and the selectivity of motion processing in human observers. In both attentional conditions, human perceptual tuning curves were considerably narrower than tuning widths typically reported for neurons in the motion sensitive area MT. This difference can be accounted for by assuming a simple model in which behavioral responses are based on sensory observations exceeding an internal decision threshold.
\end{abstract}

\section{Introduction}

At any given moment, our visual system is deluged with much more information than can be fully processed. Attention can overcome this limitation by selectively modulating the processing of sensory information according to behavioral relevance. While it is well known that attention can speed behavioral responses (Posner, 1980) and improve performance in detection and discrimination tasks (Ling and Carrasco, 2006), the mechanisms underlying these attentional benefits have been subject of substantial debate. Here, we examined the 
mechanism by which attention modulates perceptual tuning curves for direction of motion in human observers.

Response tuning curves, i.e. the average activity of individual neurons as a function of the value of a particular stimulus parameter, have been widely used to characterize the selectivity of neurons in visual and other sensory areas of the brain (Adrian, 1926). For instance, a direction tuning curve describes a neuron's response to visual motion in various directions (Dubner and Zeki, 1971; Albright, 1984). Typically, such tuning curves have a bell-shaped form and can be well fit by a Gaussian model function. Two important parameters of a tuning curve are its amplitude and its width (Butts and Goldman, 2006). While the amplitude, i.e. the difference between the strongest and weakest response, reflects the dynamic range, the width of the tuning curve represents the selectivity of the neuron.

Tuning curves have been traditionally thought of as a static property of neurons with variability only arising because of stochastic fluctuations. Recently, however, it has been reported that tuning properties of visual cortical neurons can depend on the spatiotemporal context within which a stimulus is presented (Muller et al., 1999; Dragoi et al., 2000; Yao and Dan, 2001; Felsen et al., 2002; Kohn and Movshon, 2004). Furthermore, top-down effects, such as selective attention can modulate tuning curves of sensory neurons. In this context, two different effects of attention on the tuning properties of individual neurons have been described. While one study proposes that attention decreases the width of tuning curves (Spitzer et al., 1988), the majority of experiments report that the attentional modulation consists of a multiplicative scaling of tuning curves, without substantial changes in the tuning width (Treue and Martínez-Trujillo, 1999; Martínez-Trujillo and Treue, 2004; McAdams and Maunsell, 1999; Cook and Maunsell, 2004).

Recently, a novel approach for characterizing a neuron's tuning, termed spike-triggered averaging (STA), has been developed (Chichilnisky, 2001). Here, the visual system is presented with a pixel-by-pixel contrast-modulated white noise stimulus. The spike-triggered average stimulus, or kernel, corresponds to the best linear approximation of the neuron's spatiotemporal impulse response (Dayan and Abbott, 2001). To investigate the spatiotemporal dynamics of motion selectivity the Motion Reverse Correlation (MRC) technique has been introduced (Borghuis et al., 2003; Perge et al., 2005). In this paradigm, very brief, fully-coherent motion impulses in one of several different directions are rapidly presented in a random sequence. Cross-correlating the neural response with this motion impulse sequence reveals the temporal dynamics of motion selectivity, expressed as the relative probability of observing a specific motion impulse direction at a specific point in time preceding an action potential. Based on these correlation functions direction tuning curves can be constructed over time, which allow for the analysis of motion tuning at high temporal resolution.

Here, we devised a psychophysical variant of the MRC method to obtain perceptual tuning curves for direction of motion in human observers. We asked observers to make speeded manual responses to a designated target direction embedded in a randomized sequence of motion impulses and reverse correlated these behavioral responses with the preceding motion impulse sequence. We computed time-dependent correlation functions 
showing that observers based their response on motion impulses occurring 600-250 ms before. From these correlation functions, we derived Gaussian-shaped tuning curves for direction of motion, which peaked at the designated target direction and were $\sim 17$ deg in width. This is considerably narrower than widths of tuning curves typically reported for individual direction selective neurons in area MT, suggesting that the perceptual tuning curves also reflect non-sensory, decision-related processes. We then determined the influence of attention on these perceptual tuning curves for motion direction by manipulating the amount of attention devoted to the motion impulse task. We found that full attention on the motion detection task increased the amplitude and decreased the width of perceptual tuning curves for direction of motion. In summary, this shows that, on the system's level, attention can enhance both the dynamic range and the direction selectivity of motion processing.

\section{Results}

To investigate the effects of attention on perceptual tuning curves for direction of motion we used a dual task design (Fig. 1). Observers were instructed to make speeded responses to a designated target direction embedded in a random sequence of brief motion impulses (motion impulse task) in one of 24 directions of motion, presented either to the left or the right of fixation. At the same time, they were required to count luminance changes of the fixation point. In the "full attention" condition, the luminance change in the fixation point was substantial and therefore easy to detect, such that almost full attention could be devoted to the motion detection task. In the "poor attention" condition, the luminance change was subtle, requiring more attention, and leaving only little attentional resources for the motion impulse task.

In both attentional conditions, observers' performance for counting the luminance changes of the fixation point was very high. On average, responses were correct in $85.3 \%$ and $86.2 \%$ of the trials, in the full and poor attention condition, respectively (not significantly different, paired $t$ test, $p=0.67)$. In the motion impulse task, observers correctly responded within 100-1000 ms to motion impulses in the target direction in $43.4 \%$ (full attention) and $40.5 \%$ (poor attention). This difference closely failed to reach significance (paired $t$ test, $p=0.08$ ). Observers gave, on average, $4.4 \%$ and $4.3 \%$ responses per motion impulse in the full and poor attention condition, respectively. This behavior is optimal in the sense that it equals the target probability of $4.2 \%$ ( $p>0.4$, for both conditions). The difference of $0.1 \%$ solely stems from an increased target detection performance, because the average number of responses outside the response time window was identical in both attention conditions. These results indicate that observers tended to correctly identify more targets in the full vs. the poor attention condition, without changing their overall willingness to give a response.

To compute correlograms for the motion impulse task, we cross-correlated the behavioral responses with the motion impulse sequence, separately for each attentional condition. Between -1000 and 300 ms relative to each response, the number of motion impulses were 
counted in $50 \mathrm{~ms}$ time bins, separately for each direction of motion. For each bin, these correlation functions were normalized by dividing the number of impulses in that bin by the corresponding sum of impulses across directions. Thus, the correlation functions represent the probability of observing a certain motion impulse direction at a specific point in time before (or after) a response, relative to observing any other motion impulse direction at that specific time. Correlograms of a representative observer, smoothed with a Gaussian of sigma $1 \mathrm{bin}$, are depicted in Fig. 2. The correlation functions show that the observer based his responses on motion impulses occurring 600-250 ms earlier. This correlation is strongest for motion impulses in the target direction ( $0 \mathrm{deg})$, yet the observer also responded frequently to adjacent directions $( \pm 15 \mathrm{deg})$. Interestingly, directions opposite to the target direction were less strongly suppressed than directions deviating by 90 deg from the target.

To obtain direction tuning curves, we plot the relative probabilities for all directions at the time corresponding to the peak amplitude of the most likely direction (indicated by the dashed vertical line in Fig. 2). Fig. 3 A shows the tuning curve constructed from the correlograms in Fig. 2 at time $-425 \mathrm{~ms}$. The main peak of the resulting tuning curve is centered on the target direction, and a second, much smaller peak is located at the opposite direction. We fitted these tuning curves with a sum of two Gaussians (see Methods for details). Across all observers, the designated target direction was very close to the best-fit center of the main peak, with an average absolute difference of $1.4 \mathrm{deg}$ (sd: 1.5) and 2.4 $\operatorname{deg}(\mathrm{sd}$ : 1.8), in the full and poor attention condition, respectively, indicating that the psychophysical MRC method is able to reliably extract the designated target direction. The average tuning width amounted to $17 \mathrm{deg}$.

Comparing the fitted parameters across attention conditions revealed significant differences in both the amplitude of the main peak and the width of the tuning curve. Full attention on the motion detection task increased the amplitude of the main peak by $\sim 13 \%$ (paired $t$ test, $p=0.003$ ) and decreased the width of the tuning curve by $\sim 8 \%$ (paired $t$ test, $p=0.013)$. The scatterplot in Fig. $3 \mathrm{~B}$ shows the effects for all ten observers. In addition, the average absolute difference between the best-fit center of the main peak and the true target direction was smaller in the full compared to the poor attention condition (paired $t$ test, $p=0.057$ ), indicating that observers more precisely identified the target direction in the full attention condition. We did not find significant differences between the attentional conditions for any of the other fitted parameters.

Since the average tuning width of the perceptual tuning curves (17 deg) is markedly narrower than typical tuning bandwidths of single motion-selective neurons in area MT (40-60 deg, Albright, 1984; Snowden et al., 1992; Britten, 2004), we propose that the psychophysical variant of the MRC method does not solely assess tuning for motion direction but also reflects non-perceptual, decision-related processes. In the following, we will illustrate this idea by using the results of the poor attention condition. We assume that observers solve the motion impulse task by monitoring the activity of a population of direction selective MT neurons preferring the target direction, and respond to a given motion impulse whenever the population activity exceeds an internal decision threshold (Fig. 4). More specifically, we suppose that the population tuning curve has a Gaussian 
profile, and that the variability of responses for each direction follows a Gaussian distribution with the variance equaling the mean (i.e., with a Fano factor of 1). To place the internal decision threshold, we used the percentage of correctly detected targets (40.5\%), which according to our logic, equals the number of cases the population activity evoked by targets exceeds the decision threshold. Thus, the height of the decision threshold is given by the firing rate above which the area under the Gaussian distribution of responses to targets amounts to 0.405 (green line, Fig. 4). Under assumptions of normality, false alarms to motion directions deviating from the target direction by \pm one standard deviation $(\sigma)$ should be made in $\sim 60 \%$ of the hit rate $(0.6 * 40.5 \%=24.3 \%)$, implying that the area under the Gaussian falling above the decision threshold for this direction should equal $\sim 0.24$. Indeed, varying the parameters of the population tuning curves (baseline, amplitude, width) in a physiologically plausible range yields corresponding areas near directions deviating from the target direction by the width of the perceptual tuning curve $( \pm 17.7$ deg; dashed blue line, Fig. 4). In contrast, this is never the case at \pm 40 to \pm 60 deg away from the target direction, i.e. at the typical tuning width of MT neurons (solid blue line, Fig. 4). Furthermore, using these parameters, we can also successfully predict the average number of responses per impulse $(4.30 \%)$ by determining the ratio between the area above the decision threshold and the total area. Together, these simulation results show that a simple model of population activity evaluated against an internal decision criterion can account for the narrow width of the perceptual tuning curves. Fig. 5 plots the range of physiologically plausible paramters of the population tuning curve that can account for the behavioral data. Interestingly, a comparison of the parameters of population tuning curves across attentional conditions (see Methods for details) reveals that the behavioral changes between attentional conditions can be reconciled with a multiplicative scaling of the underlying population tuning curve. This is evident from the observation that, for a constant width of the population tuning curves, performance in the full attention condition (Fig. 5, red patches) is characterized by higher amplitudes, on average, than performance in the poor attention condition (Fig. 5, blue patches). The average attentional gains found in the simulation lie approximately in between the gains reported in electrophysiological studies of attention in area MT and VIP (Treue and Martínez-Trujillo, 1999; Cook and Maunsell, 2002). Note, however, that we cannot exclude that other types of changes in the population activity might also cause similar changes in the perceptual tuning between attentional conditions.

\section{Discussion}

In this experiment, we developed a psychophysical variant of the MRC technique (Borghuis et al., 2003), a reverse correlation approach so far only used in single-unit neurophysiology, to obtain perceptual tuning curves for direction of motion in human observers. Observers were instructed to make speeded responses to a designated target direction presented within a random sequence of brief, fully coherent motion impulses in one of various directions. The correlation functions revealed that observers based their responses on directions of 
motion preceding the button press by $600-250 \mathrm{~ms}$. While the target direction had the highest correlation with the response, adjacent directions of motion were also responded to frequently. Interestingly, the opposite direction of motion also triggered a significant number of responses. The perceptual tuning curves for direction of motion constructed from the correlograms could be very well described by a sum of two Gaussian functions, with the main peak being located at the target direction and a second, much smaller peak at the opposite direction. Comparing perceptual tuning curves for conditions when the motion impulse task was performed under full vs. poor attention, revealed that attention increases the amplitude by $\sim 13 \%$ and decreases the width by $\sim 8 \%$. These results show that attention can increase the dynamic range and the selectivity of motion processing. In both attentional conditions, the widths of the perceptual tuning curves were considerably narrower than tuning widths typically found in direction-selective neurons in area MT. A conceptual model explaining this result suggests that the perceptual tuning curves might reflect, in addition to population tuning for direction of motion, non-sensory, decisionrelated variables.

In psychophysical studies the intrinsic properties of a system are only measurable indirectly. A successful method for recovering the internal tuning properties of human observers has been the use of classification images (Ahumada Jr. and Lovell, 1971; Eckstein et al., 2002), a psychophysical variant of the STA approach in single unit electrophysiology. Here, white noise stimuli, in some trials superimposed on a target signal, are presented to human subjects in detection (Neri and Heeger, 2002) or discrimination tasks (Mareschal et al., 2006). The noise stimuli of each trial are sorted according to the behavioral response (correct/incorrect) of the subject, averaged on a pixel-by-pixel basis and finally subtracted from each other to yield the best linear estimate of the observer's template or kernel (Ahumada Jr., 2002). While this approach can very well be applied to stationary stimuli it is not suited to investigate perceptual tuning curves for direction of motion since the dynamics of the stimulus cannot be captured by pixel-wise averaging. To study perceptual tuning curves in the motion domain, we developed a variant the MRC technique, a reverse correlation method usually applied to moving stimuli in single-unit electrophysiology, for use in psychophysics. The psychophysical MRC approach yields, already after a relatively small number of trials, smooth correlation functions, with the peak of the perceptual tuning curves precisely indicating the target direction. The effectiveness and the straightforward use of the psychophysical variant of the MRC method makes it a very promising tool to recover characteristics of human motion tuning in a large variety of tasks.

For individual motion-sensitive neurons in area MT it has been shown that tuning curves obtained by the MRC method are very well comparable to classical tuning curves obtained by presenting moving stimuli for longer durations (Borghuis et al., 2003; Perge et al., 2005). Likewise, using the psychophysical variant of the MRC technique we find that, for each individual observer, the main peak of the recovered perceptual tuning curve corresponds very closely to the assigned target direction, while adjacent directions of motion are less strongly represented. The width of the perceptual tuning curves, however, is considerably smaller (17 deg) than the typical tuning width of individual MT neurons (50 deg). A model assuming that the psychophysical variant of the MRC method mea- 
sures the tuning of a typical neuronal MT population truncated at a decision criterion can very well explain this difference for a wide range of physiologically plausible parameters. Thus, the simulations show that the perceptual tuning curves might indeed reflect both sensory and decision-related variables. A further major difference between physiological and perceptual tuning curves concerns the second peak in the perceptual tuning curves which arises because observers make a significant number of responses to directions of motion opposite to the target direction. Here, it seems likely that observers base these judgments on the orientation of the motion vector rather than on the specific direction of motion. An important role of orientation in motion processing has been postulated for fast motion, which should create, due to the temporal integration characteristics of the visual system, a "motion streak" oriented in the motion direction (Geisler, 1999; Burr and Ross, 2002). Given the very brief motion impulses, it might be very well the case that estimates of velocity are less reliable than the estimates of the orientation of motion streaks (Burr, 1980; Lorenceau et al., 1993).

Since the tuning curves obtained by the MRC method measure relative probabilities of observing a particular direction of motion at a specific time, the data points constituting a single tuning curve cannot vary independently. An increased probability for one direction necessarily leads to a decreased probability for at least one other direction, because the data values have to add to 1 . It is therefore important to emphasize that increased gains do not automatically have to covary with decreased tuning bandwidths, because any increases could also be counterbalanced by decreases in the baseline response. We do not find any systematic changes in the baseline parameter across attentional conditions. Furthermore, a purely multiplicative scaling of perceptual tuning curves between conditions would have no effect on the perceptual tuning curves because the normalization would cancel out any simple scaling. Since we find significant changes of both amplitude and tuning bandwidth we conclude that, on the system's level, we observe combined effects of signal enhancement and noise exclusion.

Previous single-unit studies of motion selective neurons in the middle temporal area (MT) have reported that both spatial and feature-based attention exert a multiplicative enhancement on tuning curves for direction of motion without a change in the tuning bandwidth (Treue and Martínez-Trujillo, 1999; Cook and Maunsell, 2004). Similar results have been reported for different visual stimulus dimensions and in different areas of visual cortex (V1, V4) (McAdams and Reid, 2005; McAdams and Maunsell, 1999). Our simulation shows that, in principle, the observed behavioral effects of both an increased amplitude and a decreased tuning width can be accounted for by a purely multiplicative scaling of the underlying population tuning curves, however, we cannot exclude that other neuronal mechanisms can achieve the same behavioral output. Furthermore, it should be noted that single neuron tuning curves do not always translate linearly into tuning curves of the whole population. For feature-based attention, e.g. attention directed to a particular direction of motion, it has been shown that, while single neuron tuning curves are multiplicatively modulated by attention, the population tuning increases in selectivity (Martínez-Trujillo and Treue, 2004). It is very well reasonable, that observers used a combination of spatial and feature-based attention in the motion impulse task. 
The psychophysical studies investigating attentional influences on sensory tuning yield inconsistent results (Neri and Levi, 2006), with some reporting a sharpening (Yeshurun and Carrasco, 1998; Dosher and Lu, 2000; Lee et al., 1999) while others show either gain changes (Lu and Dosher, 1998; Eckstein et al., 2002; Murray et al., 2003) or no effect of attention on perceptual tuning curves (Neri, 2004; Talgar et al., 2004). Neri and Levi (2006) argue that a purely multiplicative scaling of population tuning curves would not necessarily be observable in the perceptual tuning curves reconstructed from the observers' behavior because psychophysical reverse correlation can recover the shape of the perceptual filter only up to a multiplicative scaling factor which depends on more than just the underlying gain (Ahumada Jr., 2002). Hence, it is very difficult, based on these psychophysical studies alone, to estimate the size of the gain change relative to the sharpening of the tuning curves.

In this work, we have applied a behavioral variant of the MRC approach, a reverse correlation technique used to estimate tuning curves in single unit electrophysiology, to psychophysical data in order to obtain perceptual tuning curves for direction of motion. The perceptual tuning curves for direction of motion can very well be described by a sum of two Gaussians whose main peak is located very close to the target direction while a second, much smaller peak occurs around the opposite direction. Furthermore, we show that attention increases the amplitude and decreases the width of the perceptual tuning curves, demonstrating that, on the system's level, attention can increase the dynamic range and the selectivity of tuning curves for direction of motion. Using a conceptual model of MT responses, we propose that our results reflect the population activity for direction of motion truncated at a non-sensory, decision-related threshold.

\section{Methods}

\section{Apparatus}

The experiment was conducted in a dimly illuminated and quiet room. Stimuli were presented on a VGA monitor (Lacie, electron22blueIV) operated at a refresh rate of $85 \mathrm{~Hz}$ and a spatial resolution of $40 \mathrm{px} / \mathrm{deg}$. Stimulus presentation and recording of responses was controlled by custom-made software running on an Apple Power Mac G4. Subjects placed their head on a chin-rest or a bite-bar positioned $114 \mathrm{~cm}$ from the monitor. During the trials, eye-movements were monitored using an infrared eye-tracking system (ISCAN ETL-200).

\section{Stimulus}

On each trial, subjects fixated a central fixation square $(0.05 \times 0.05$ deg $)$ presented on a black background (luminance $0.05 \mathrm{~cd} / \mathrm{m}^{2}$ ). A circular aperture (3.75 deg diameter), containing moving gray dots (luminance $5.5 \mathrm{~cd} / \mathrm{m}^{2}$ ) was shown 3.75 deg to either the left or the right of the fixation square. The position of the circular aperture was alternated from trial to trial. Before trial start, the dots (density: 15 dots per $\mathrm{deg}^{2}$, dot size: $0.2 \mathrm{deg}$ ) 
were stationary to indicate the stimulus location to the observer. After initiation of the trial, the dots moved in a random sequence of brief (94 ms), fully coherent motion impulses in one of 24 different directions, sampled every $15 \mathrm{deg}$. Individual motion impulses were separated by a blank screen of $47 \mathrm{~ms}$. One trial was composed of 106 motion impulses and lasted 15 seconds. During the course of the trial, the fixation square could briefly change its luminance up to 3 times (for a duration of $120 \mathrm{~ms}$ ). In two different conditions, run in different sessions, the luminance was reduced by $75 \mathrm{~cd} / \mathrm{m}^{2}$ or $40 \mathrm{~cd} / \mathrm{m}^{2}$, respectively. The difficulty of the smaller luminance change was chosen to yield about $75-80 \%$ correct responses according to a pilot experiment in which we systematically varied the intensity of the change.

\section{Paradigm}

A dual-task design was used. For the primary task, subjects were instructed to count the number of luminance changes of the fixation point and to indicate, at trial end, the number of changes by pressing one of the keys $0-3$ on the number pad of the keyboard. After each trial, subjects received auditory feedback depending on performance in this task. Additionally, subjects were instructed to covertly direct their attention to the peripheral $\mathrm{RDP}$ in order to make speeded responses (using key ' $\mathrm{H}$ ') to motion impulses moving in a designated target direction (secondary task). Target directions were either rightward (half of the subjects) or leftward motion (other half of the subjects). Each key press for the secondary task was acknowledged by a sound. Key presses within 100-1000 ms after a motion impulse in the target direction were counted as hits. After the last motion impulse in each trial, a blank screen was presented for $550 \mathrm{~ms}$ to allow for responses to the last few motion impulses. At trial end, a summary of the subjects' performance for the current trial was displayed in the upper left corner of the screen, informing them about the number of hits, the number of targets, and the number of responses outside the response time window.

The experiment was run in two sessions on different days. Each session lasted for about $1.5 \mathrm{~h}$ and started with a practice block (32 trials) which was not included in the data analysis. The experiment consisted of 3 blocks of 32 trials each. The two sessions differed in the magnitude of the luminance change in the fixation point. We termed the condition with substantial luminance changes "full attention" condition, because the size of the luminance change left almost full attentional resources for the motion impulse task. In contrast, the condition with the smaller luminance change was called "poor attention" condition because counting the luminance changes left less attentional resources for the motion impulse task. The order in which subjects performed the two attentional conditions was counterbalanced across subjects. 16 subjects participated in the experiment (age 15-34, 12 females).

\section{Data analysis}

For each attention condition separately, we calculated correlation functions and tuning curves based on only those trials with correct performance in the primary task. Analogously to the MRC method in single-unit physiology (Borghuis et al., 2003), we determined the 
onset times and directions of all motion impulses preceding and following each response by -1000 to $300 \mathrm{~ms}$. For each direction of motion separately, these motion impulses were sorted into an array according to their specific onset times (50 ms bin width). Finally the number of motion impulses in each bin was divided by the total number of motion impulses in that bin. Thus, the correlation functions express the probability that a motion impulse in a particular direction occurred at a specific time preceding a response, relative to of observing motion impulses from any of the other directions. Correlation functions range from 0 , indicating zero probability of observing a certain motion impulse direction at that specific time before a response, to 1 , indicating that each response was preceded by that direction of motion at that moment in time. The level of equal probability corresponds to $1 / \mathrm{n}$, with $\mathrm{n}$ representing the number of motion directions (in our case: $1 / 24=0.042$ ). Correlation functions were smoothed with a Gaussian (sigma of 1 bin). The amount of noise can be assessed by computing the standard deviation of the correlation functions in the time bins following the response (these values should ideally be $1 / n$, since responses cannot be influenced by future stimuli). A criterion of \pm 2 standard deviations was used to define significant deviations of individual correlograms from the equal probability level.

Tuning curves were constructed for each observer by plotting the correlation functions at the time of the peak of the most likely direction as a function of direction of motion. These tuning curves were fitted with a sum of two Gaussians, shifted by 180 deg and differing in amplitude, but not in width (additional free parameters for the location of the second center or the width do not significantly improve the amount of explained variance):

$$
p(x)=B+A_{1} * \exp -0.5\left(\frac{x-C_{1}}{W}\right)^{2}+A_{2} * \exp -0.5\left(\frac{x-\left(C_{1} \pm 180\right)}{W}\right)^{2},
$$

where $x$ are the 24 directions used in the experiment, $B$ is the baseline, $A_{1}$ and $A_{2}$ correspond to the amplitudes, and $C_{1}$ represents the main peak of the Gaussians. To obtain the location of the second peak, $180 \mathrm{deg}$ was subtracted or added to main peak, in case the subject was assigned leftwards or rightwards targets, respectively. Subjects were only included in all further analysis if this model could account for, at least, 95\% of the variance in the data, adjusted by the degrees of freedom. Based on this criterion, 6 subjects were excluded from the analysis. Parameters of the tuning curves were compared across conditions using paired-sample $t$ tests.

\section{Model of population tuning}

For the model examining the differences between neuronal and perceptual tuning curves, we computed mean responses for the idealized population tuning curves using

$$
r(x)=B+A * \exp \left(-0.5 *(x-C)^{2} / W^{2}\right)
$$

where $x$ are the 24 directions used in the experiment, $C$ is the preferred direction (0), $B$ is the response to the antipreferred direction, $A$ is the maximal response modulation, and $W$ is the selectivity of the population. To compare model predictions to the behavioral 
data we systematically varied the parameters $B(0-20 \mathrm{sp} / \mathrm{s}), A(10-160 \mathrm{sp} / \mathrm{s})$, and $W(40-$ $70 \mathrm{deg})$. We assumed that the responses along the y-axis for each direction are Gaussian distributed, with the variance of the Gaussian equaling the mean (i.e., with a Fano factor of 1). To place the decision threshold, we calculated, for each attentional condition, the firing rate elicited by target presentations above which the area under the Gaussian corresponded to the observed hit rate. We next determined the area under the Gaussian that should lie above the decision criterion for directions deviating from the target direction by one sigma. Given that the ratio between the ordinate at $\sigma$ and the peak of a Gaussian is $\sim 0.6$ we simply multiplied the hit rate by this factor. We considered the behavioral data well explained if both the area under the Gaussian at the perceptual tuning width did not differ more than $\pm 10 \%$ from $60 \%$ of the hit rate, and if the area above the threshold (summed across directions) divided by the total area $(24 * 1))$ did not deviate more than $\pm 10 \%$ from the average number of responses per impulse. The average attention gain was calculated, separately for each width of population tuning, by plotting the data points of each possible pair of tuning curves (i.e., poor attention vs. full attention) satisfying the above described conditions and by determining the slope of the best-fit line using linear regression analysis. Finally, the obtained regression coefficients were averaged across all possible pairs.

\section{Acknowledgments}

This work was supported by BMBF Grant 01GQ0433.

\section{References}

Adrian, E. D. (1926). The impulses produced by sensory nerve endings. Journal of Physiology, 61:49-72.

Ahumada Jr., A. J. (2002). Classification image weights and internal noise level estimation. Journal of Vision, 2:121-131.

Ahumada Jr., A. J. and Lovell, J. (1971). Stimulus features in signal detection. Journal of the Acoustical Society of America, 49(6):1751-1756.

Albright, T. D. (1984). Direction and orientation selectivity of neurons in visual area MT of the macaque. Journal of Neurophysiology, 52(6):1106-1130.

Borghuis, B. G., Perge, J. A., Vajda, I., van Wezel, R. J., van de Grind, W. A., and Lankheet, M. J. (2003). The motion reverse correlation (MRC) method: a linear systems approach in the motion domain. Journal of Neuroscience Methods, 123(2):153-166.

Britten, K. H. (2004). The middle temporal area: motion processing and the link to perception. In Chalupa, L. M. and Werner, J. F., editors, The Visual Neurosciences, pages 1203-1216. MIT Press, Cambridge, MA. 
Burr, D. C. (1980). Motion smear. Nature, 284(5752):164-165.

Burr, D. C. and Ross, J. (2002). Direct evidence that "speedlines" influence motion mechanisms. Journal of Neuroscience, 22(19):8661-8664.

Butts, D. A. and Goldman, M. S. (2006). Tuning curves, neuronal variability, and sensory coding. PLoS Biology, 4(4):e92.

Chichilnisky, E. J. (2001). A simple white noise analysis of neuronal light responses. Network: Computation in Neural Systems, 12(2):199-213.

Cook, E. P. and Maunsell, J. H. (2002). Attentional modulation of behavioral performance and neuronal responses in middle temporal and ventral intraparietal areas of macaque monkey. Journal of Neuroscience, 22(5):1994-2004.

Cook, E. P. and Maunsell, J. H. (2004). Attentional modulation of motion integration of individual neurons in the middle temporal visual area. Journal of Neuroscience, 24(36):7964-7977.

Dayan, P. and Abbott, L. F. (2001). Theoretical Neuroscience. Computational and Mathematical Modeling of Neural Systems, chapter Neural encoding II: Reverse correlation and visual receptive fields, pages 45-85. MIT Press.

Dosher, B. A. and Lu, Z. L. (2000). Mechanisms of perceptual attention in precuing of location. Vision Research, 40(10-12):1269-1292.

Dragoi, V., Sharma, J., and Sur, M. (2000). Adaptation-induced plasticity of orientation tuning in adult visual cortex. Neuron, 28:287-298.

Dubner, R. and Zeki, S. M. (1971). Response properties and receptive fields of cells in an anatomically defined region of the superior temporal sulcus in the monkey. Brain Research, 35:528-532.

Eckstein, M. P., Shimozaki, S. S., and Abbey, C. K. (2002). The footprints of visual attention in the Posner cueing paradigm revealed by classification images. Journal of Vision, 2(1):25-45.

Felsen, G., Shen, Y.-s., Yao, H., Spor, G., Li, C., and Dan, Y. (2002). Dynamic modification of cortical orientation tuning mediated by recurrent connections. Neuron, 36:945-954.

Geisler, W. S. (1999). Motion streaks provide a spatial code for motion direction. Nature, 400(6739):65-69.

Kohn, A. and Movshon, J. A. (2004). Adaptation changes the direction tuning of macaque mt neurons. Nature Neuroscience, 7(7):764-772.

Lee, D. K., Itti, L., Koch, C., and Braun, J. (1999). Attention activates winner-take-all competition among visual filters. Nature Neuroscience, 2(4):375-381. 
Ling, S. and Carrasco, M. (2006). Sustained and transient covert attention enhance the signal via different contrast response functions. Vision Research, 46(8-9):1210-1220.

Lorenceau, J., Shiffrar, M., Wells, N., and Castet, E. (1993). Different motion sensitive units are involved in recovering the direction of moving lines. Vision Research, 33(9):1207-1217.

Lu, Z. L. and Dosher, B. A. (1998). External noise distinguishes attention mechanisms. Vision Research, 38(9):1183-1198.

Mareschal, I., Dakin, S. C., and Bex, P. J. (2006). Dynamic properties of orientation discrimination assessed by using classification images. Proceedings of the National Academy of Sciences, 103(13):5131-5136.

Martínez-Trujillo, J. C. and Treue, S. (2004). Feature-based attention increases the selectivity of population responses in primate visual cortex. Current Biology, 14(9):744-751.

McAdams, C. J. and Maunsell, J. H. (1999). Effects of attention on orientation-tuning functions of single neurons in macaque cortical area V4. Journal of Neuroscience, 19(1):431441.

McAdams, C. J. and Reid, R. C. (2005). Attention modulates the responses of simple cells in monkey primary visual cortex. Journal of Neuroscience, 25(47):11023-11033.

Muller, J. R., Metha, A. B., Krauskopf, J., and Lennie, P. (1999). Rapid adaptation in visual cortex to the structure of images. Science, 285(5432):1405-1408.

Murray, R. F., Sekuler, A. B., and Bennett, P. J. (2003). A linear cue combination framework for understanding selective attention. Journal of Vision, 3(2):116-145.

Neri, P. (2004). Estimation of nonlinear psychophysical kernels. Journal of Vision, 4(2):8291.

Neri, P. and Heeger, D. J. (2002). Spatiotemporal mechanisms for detecting and identifying image features in human vision. Nature Neuroscience, 5(8):812-816.

Neri, P. and Levi, D. M. (2006). Receptive versus perceptive fields from the reversecorrelation viewpoint. Vision Research, 46(16):2465-2474.

Perge, J. A., Borghuis, B. G., Bours, R. J., Lankheet, M. J., and van Wezel, R. J. (2005). Temporal dynamics of direction tuning in motion-sensitive macaque area MT. Journal of Neurophysiology, 93(4):2104-2116.

Posner, M. I. (1980). Orienting of attention. Quarterly Journal of Experimental Psychology, $32(1): 3-25$. 
Snowden, R. J., Treue, S., and Andersen, R. A. (1992). The response of neurons in areas $\mathrm{V} 1$ and MT of the alert rhesus monkey to moving random dot patterns. Experimental Brain Research, 88(2):389-400.

Spitzer, H., Desimone, R., and Moran, J. (1988). Increased attention enhances both behavioral and neuronal performance. Science, 240(4850):338-340.

Talgar, C. P., Pelli, D. G., and Carrasco, M. (2004). Covert attention enhances letter identification without affecting channel tuning. Journal of Vision, 4(1):22-31.

Treue, S. and Martínez-Trujillo, J. C. (1999). Feature-based attention influences motion processing gain in macaque visual cortex. Nature, 399(6736):575-579.

Yao, H. and Dan, Y. (2001). Stimulus timing-dependent plasticity in cortical processing of orientation. Neuron, 32(2):315-323.

Yeshurun, Y. and Carrasco, M. (1998). Attention improves or impairs visual performance by enhancing spatial resolution. Nature, 396(6706):72-75. 
Figure 1:

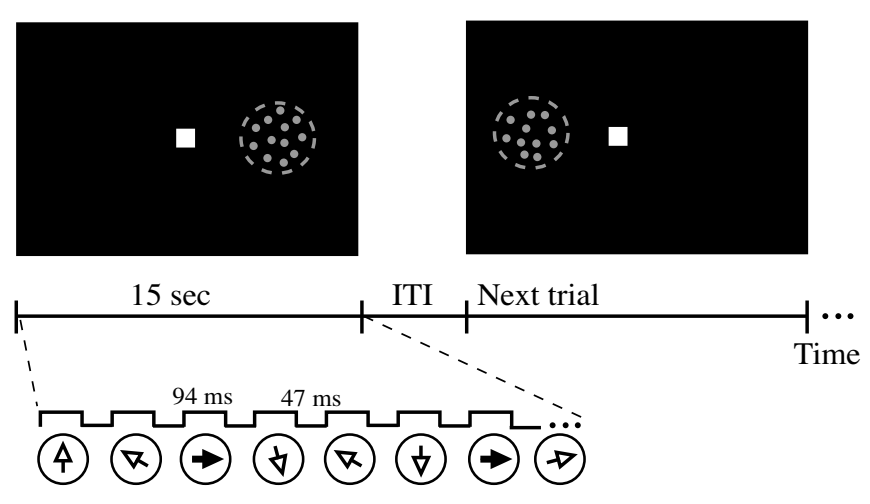


Figure 2:
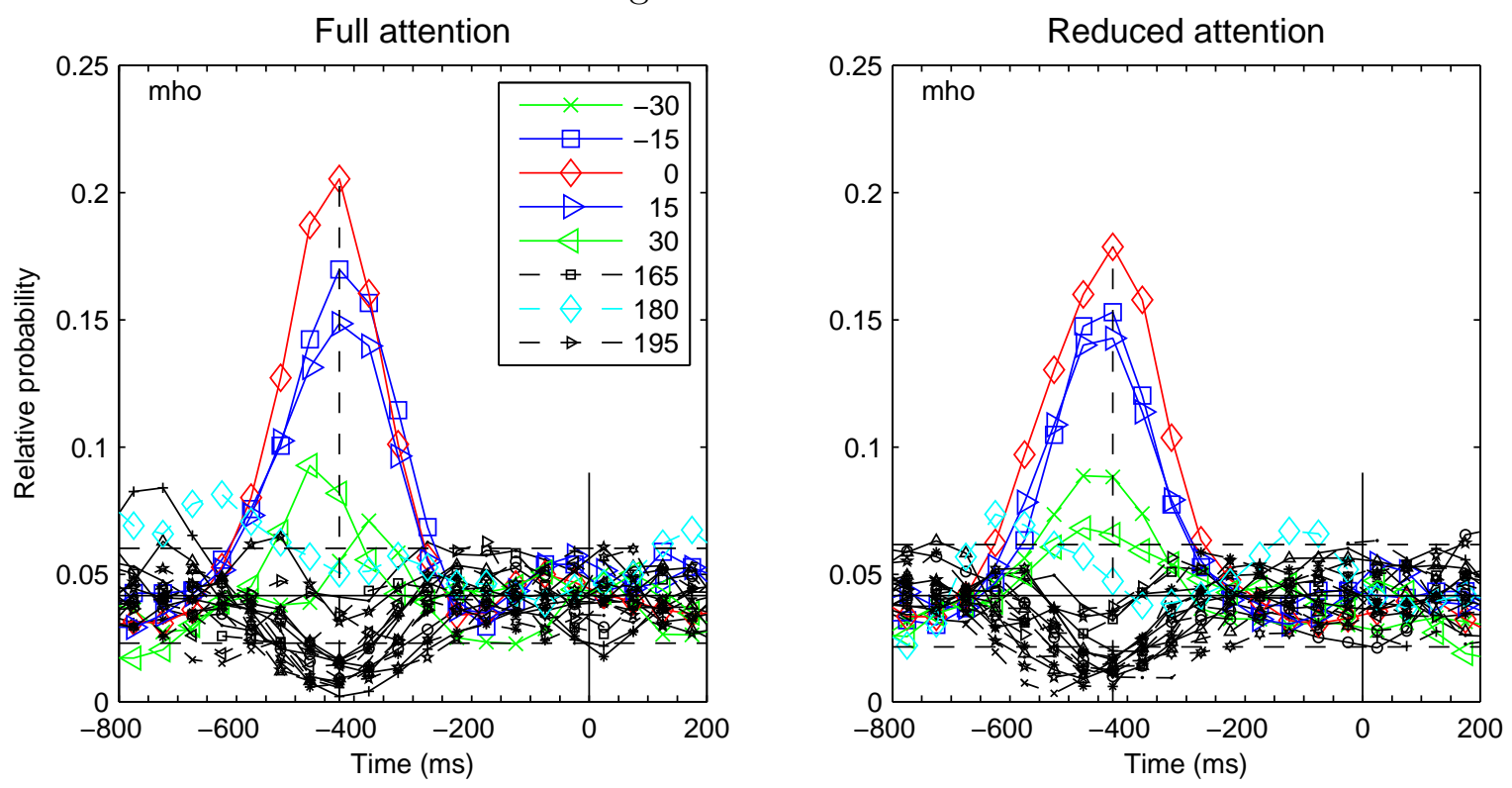
Figure 3:
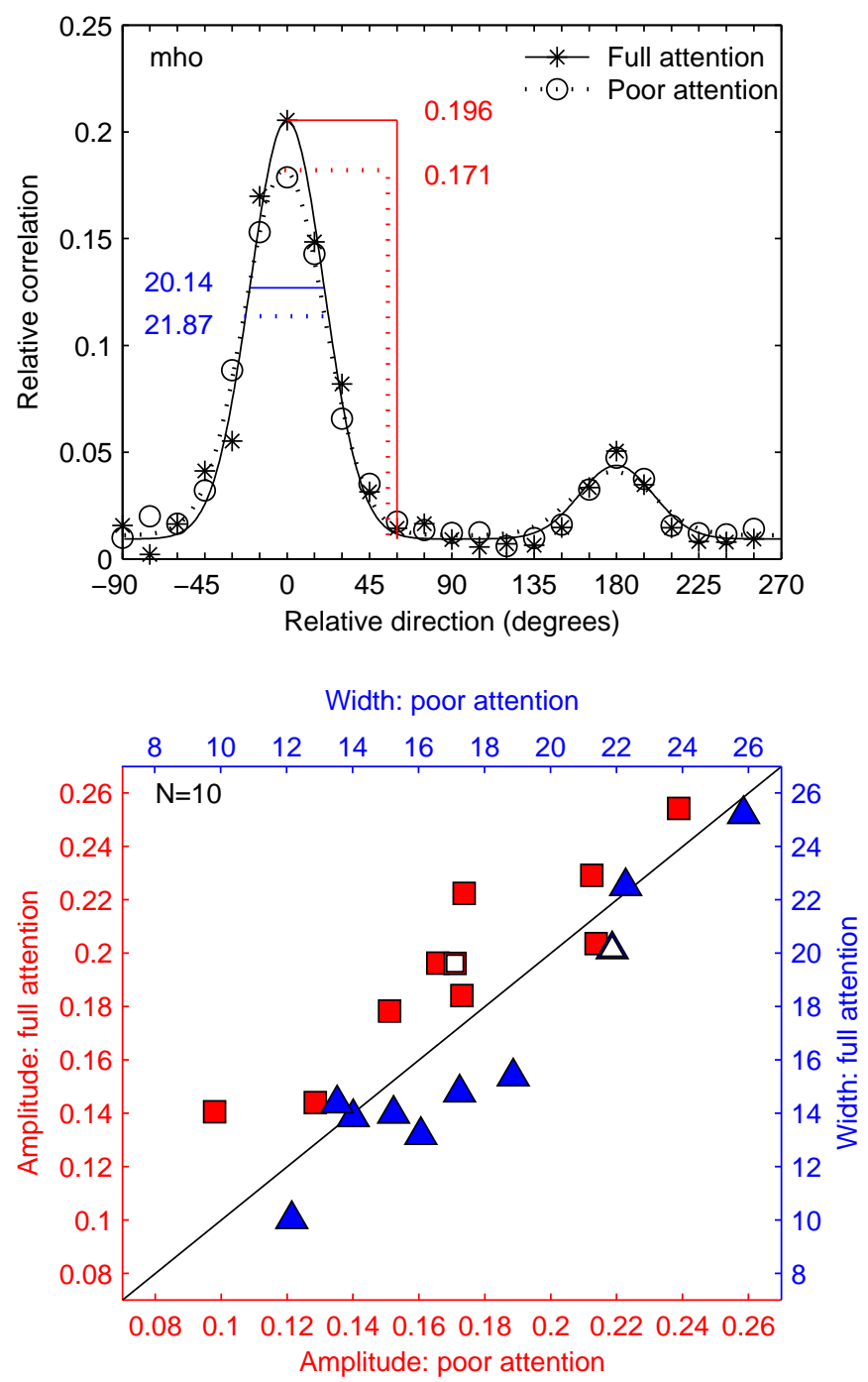
Figure 4:

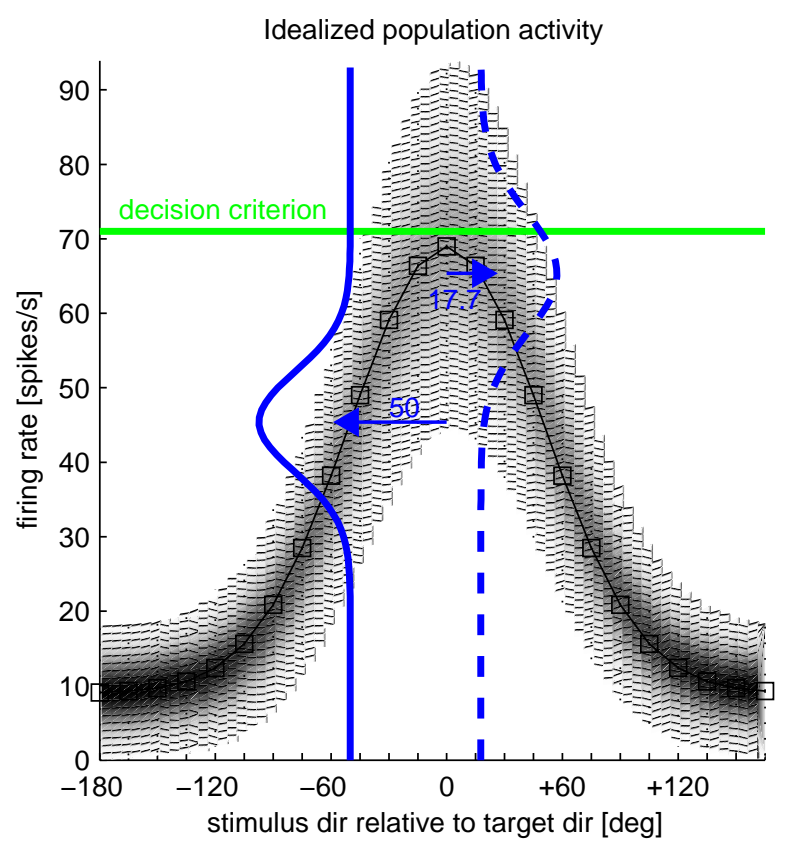


Figure 5:
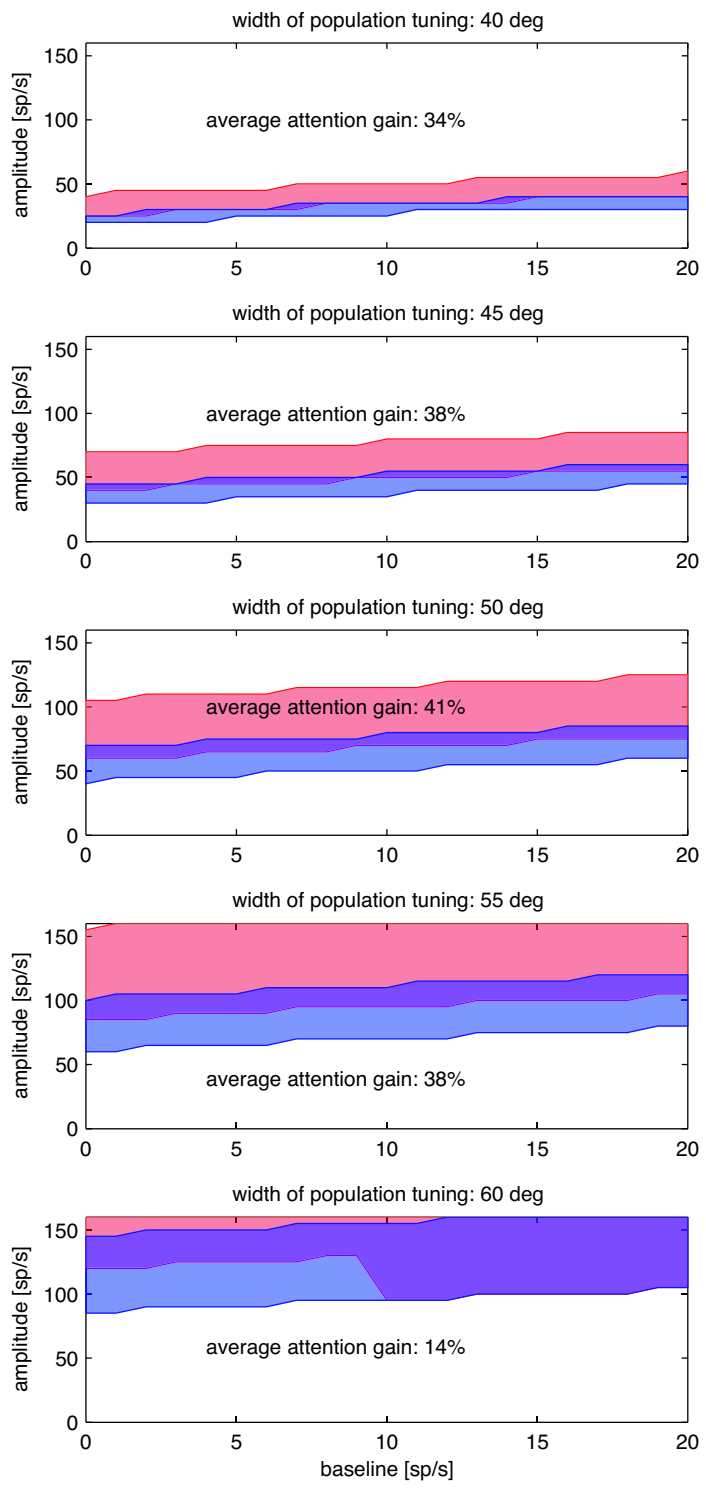
Fig. 1. Behavioral task. Observers performed a dual task. The primary task consisted of counting the number of luminance changes in the fixation point and reporting the outcome (0-3 changes) at the end of each trial (15 s duration). For the secondary task, observers were instructed to make speeded responses to a designated motion direction embedded in a random sequence of brief, fully coherent motion impulses. In this example, the target direction is rightward motion, which is indicated by the black arrow head pointing to the right in the stream of motion impulses. The dot pattern containing the motion impulse sequence was presented to the left or the right of the fixation point with the side changing systematically from trial to trial to avoid effects of adaptation. In two different conditions, the luminance change in the fixation point was either substantial or subtle, thus leaving almost "full" or only "poor" attentional resources for the motion impulse task. 
Fig. 2. Correlation functions for a representative observer obtained by cross-correlating the behavioral responses with the motion impulse sequence in conditions of full (A) and poor attention (B). Each line represents the time course of the probability of observing a particular direction of motion relative to observing any other direction of motion, with time 0 being the time of the behavioral response (solid vertical line). The solid horizontal line indicates the equal probability level $(1 / 24)$, the horizontal dashed lines indicate \pm 2 standard deviations obtained from the non-correlated part of the correlation functions (see Methods). The correlation functions in both conditions change significantly between 600$250 \mathrm{~ms}$ preceding the response, indicating that observers based their response on motion impulses occurring in this time window. Correlations are strongest for the motion impulses in the target direction (here: $0 \mathrm{deg}$, red line), and decrease as directions deviate from the target motion impulse. Remarkably, correlations for the direction opposite to the target direction (cyan line) are not as strongly suppressed as directions differing only by 90 deg. 
Fig. 3. Perceptual tuning curves. A. Perceptual tuning curves for direction of motion obtained from the data shown in Fig. 2 at the time of the highest peak in those correlation functions $(-425 \mathrm{~ms})$. Correlations in the "full" attention condition are plotted as asterisks, whereas correlations in the "poor" attention condition are indicated by open circles. Tuning curves were fit using a sum of two Gaussian functions, shifted by $180 \mathrm{deg}$. The fit is depicted as a solid vs. dotted trace for the "full" and "poor" attention condition, respectively. In both conditions, the main peak corresponds to a direction of motion very close to the target direction, while a second, much smaller peak is located at the opposite direction. The tuning width amounts to $17 \mathrm{deg}$ (averaged across the two conditions). The amplitude of the fitted model function is higher (0.2 vs. 0.17 ) and the width is narrower (16.3 vs. 17.7) in the "full" compared to the "poor" attention condition. B. Scatter plot of attention effects on amplitude (squares) and width (triangles) of the perceptual tuning curves for all observers. The data of the observer shown in A are marked by open symbols. For the majority of observers, attention increases the amplitude and decreases the width of the perceptual tuning curves for direction of motion. On average, the increase in amplitude amounts to $\sim 13 \%$ whereas the decrease in tuning width is about $\sim 8 \%$. 
Fig. 4. Schematic model accounting for the differences in tuning width between perceptual and neuronal tuning curves, illustrated using data obtained in the poor attention condition. We hypothesized that observers reported target presence if the activity of a neuronal population preferring the target direction exceeded an internal decision threshold. Plotted is an idealized population response across the 24 directions presented in the experiment (baseline $9 \mathrm{sp} / \mathrm{s}$, amplitude $60 \mathrm{sp} / \mathrm{s}$, width $50 \mathrm{deg}$ ). The responses for each direction are assumed to be Gaussian distributed along the y-axis with the mean equaling the variance (dotted lines correspond to three standard deviations). The green line represents the decision threshold which will be crossed in $40.5 \%$ of the target presentations, given the distribution of neuronal responses to the target stimulus. At the width of the neuronal population tuning curve ( $\pm 50 \mathrm{deg}$, blue leftwards pointing triangle), a very small fraction of responses crosses this threshold (blue solid line). In contrast, at directions deviating from the target direction by the width of the perceptual tuning curve $( \pm 17.7$ deg, blue rightwards pointing triangle), the percentage of responses crossing the threshold correspond well to the expected percentage of false alarms ( $0.6 * 40.5 \%$, dashed blue line). Furthermore, the summed area above the decision threshold amounts to $\sim 4.6 \%$ of the total area, corresponding very closely to the experimentally obtained number of responses per impulse $(4.3 \%)$. 
Fig. 5. Combinations of model parameters which both predict the experimental results and are physiologically plausible. We evaluated the above described model for combinations of parameters values for baseline, amplitude, and width (see Methods). Plotted are those combinations that correctly predict (within $\pm 10 \%$ ) both the number of false alarms at the width of the perceptual tuning curve and the overall number of responses per impulse. Red patches refer to parameter combinations obtained in the full attention condition, blue patches to those in the poor attention condition. Interestingly, the behavioral differences between the two attentional conditions seem to be in agreement with a multiplicative scaling of underlying population tuning curves without changes in the width. 


\subsection{Object-based attention modulates activity of single neu- rons in primate visual cortex}

Attention cannot only be allocated to spatial locations or visual features, but also to whole objects. So far, two characteristics of object-based attention have been described. First, attention to one part of an object results in an improved performance for targets presented at an uncued location within the same object compared to an equidistant location outside the object (Egly et al., 1994). Recent neurophysiological (Roelfsema et al., 1998) and fMRI studies (Müller and Kleinschmidt, 2003) have demonstrated that the neuronal mechanism underlying this behavioral benefit seems to be an automatic attentional modulation of neurons processing information at retinotopic locations corresponding to all different parts of the object. Second, behavioral studies have shown that subjects are better in judging two attributes if they belong to the same object compared to different objects ("same-object advantage"). These findings led to the key prediction of object-based attentional theories: Attending to a single feature of an object results in an attentional enhancement of the processing of all features of the object, even if they are irrelevant. In line with this prediction, an fMRI study has demonstrated that attending to a moving face enhances the neuronal activity not only in face-selective but also in motion sensitive areas of the cortex (O’Craven et al., 1999).

In this experiment, we tested the later prediction of object-based attention by recording extracellular activity of single units in the motion sensitive area MT, which is rather insensitive to the color of a stimulus (Felleman and Van Essen, 1991; Ungerleider and Mishkin, 1982). Consistent with object-based theories of attention, we find that neuronal responses in area MT are enhanced even if the animals attend to the color of a moving object. Furthermore, attending to one feature of the object leads to a globally enhanced processing of all features of the object across the entire visual field. 


\title{
Object-based Attention Modulates Stimulus Representa- tions Across the Visual Field in Macaque Cortical Area MT
}

Steffen Katzner ${ }^{1,2}$, Laura Busse ${ }^{1,2} \&$ Stefan Treue ${ }^{1,2,3}$

\author{
${ }^{1}$ Cognitive Neuroscience Laboratory, German Primate Center, Göttingen, Germany \\ ${ }^{2}$ Bernstein Center for Computational Neuroscience, Göttingen, Germany \\ ${ }^{3}$ Department of Biology, University of Göttingen, Germany
}

Visual scenes typically contain much more information than our brains can fully process. To focus our processing resources, we can direct attention to a location in space ${ }^{1}$, to non-spatial stimulus features, like a specific color or motion direction ${ }^{2}$, or even to whole objects ${ }^{3}$. Many single-unit studies in awake behaving primates have demonstrated neural correlates of spatial and feature-based attention in various areas of visual cortex ${ }^{4-7}$. Yet, no such studies have tested the central prediction made by theories of object-based attention: that attention directed to a single feature of an object will transfer to the other features of the same object, thereby enhancing the processing of even those features that are currently irrelevant. Here we document for the first time the neural correlate of such a transfer and its global effect on the processing of unattended stimuli in single-unit recordings from area MT in the primate visual cortex. Our results provide important insights into the mechanisms underlying the distribution of attention within and between visual objects, and demonstrate that even focused attention affects the representation of the whole visual scene.

To investigate the neuronal correlates of object-based attention, we recorded extracellularly 
from individual neurons in the middle temporal area (MT) of the visual cortex of two macaque monkeys while they were attending either to the color or the direction of motion of a random dot pattern (RDP). Because MT neurons are highly selective for processing of motion direction and speed, rather than color ${ }^{8,9}$, MT activity provides a sensitive and reliable measure of motion signal processing under different attentional conditions. Hence, any object-based attentional transfer between visual features should be reflected in an enhanced processing of the motion signal, even if attention is directed to the color of a moving stimulus.

Our display consisted of two colored RDPs moving either in the preferred or in the null direction of the neuron under study. One RDP was placed inside the classical receptive field (RF) of the neuron, the other one outside at an equal eccentricity in the opposite visual hemifield. In separate blocks of trials, the monkeys were cued to attend either to the motion direction or to the color in one of the two RDPs (the target stimulus) in order to perform a direction or a color task, respectively. In the direction task, the monkeys had to detect a brief change in the direction of motion of the target. In contrast, in the color task, the monkeys were required to detect a brief change in the color (Fig. 1). To ensure that the monkeys attended to the cued feature of the target, we used different types of distractors, randomized in time and order (see Supplementary Figure 1). For instance, in the direction task, the target could also change its color, or the irrelevant stimulus could change its direction of motion or color. To further ensure that the monkeys were accurately following the attentional instructions, we only included those individual blocks of trials in all subsequent analyses, for which statistical evaluation confirmed that the behavior reliably followed the attentional instructions. These results are illustrated by Supplementary Figure 2. 
As a first test for a transfer of attention between visual features, we compared the attentional enhancement of firing rates caused by directing attention to the stimulus inside the RF between conditions, in which the animals attended to the motion direction versus the color of the RF stimulus. Consistent with theories of object-based attention, we found that the attentional enhancement in the color task was not different from that in the direction task $(p=0.86$, paired $t$ - test; see also Supplementary Figure 4). Moreover, average single-unit firing rates in these two conditions were perfectly correlated $\left(r=0.99, p=2.2^{-16}\right)$. While this finding is in accord with an object-based attentional transfer from color to motion, explanations based on spatial attentional effects (illustrated by Supplementary Figure 3) cannot be ruled out for this comparison.

To dissociate the spatial focus of attention from the visual object driving the neurons under study, we compared neuronal activity between conditions in which the animals' attention was directed to the motion or the color of a preferred- versus null-direction stimulus outside the RF, while the stimulus inside always moved in the preferred direction of the recorded neuron. Data from a representative MT neuron are shown in Fig. 2A. The dotted traces show the well-known neural correlate of feature-based attention in the direction task ${ }^{10,11}$, i.e., attending to the preferred (dotted red trace) versus null direction (dotted black trace) outside the RF increased responses to the irrelevant stimulus in the RF. Using the color task allowed us to directly test for the presence of object-based attention: We found that firing rates were higher if attention was directed to the color of a preferred- (solid red trace) versus null-direction stimulus (solid black trace) outside the RF. Fig. 2B documents the same effects when pooling across our sample of 58 neurons. The distribution of attentional modulation indices for all neurons studied is shown in Fig. 2C. Attending 
to the preferred versus null direction outside the RF increases responses by $5.7-18.3 \%$ (95-\% confidence interval) in the direction task (gray bars) $(p=0.00021$, one sample $t$ - test). When attention is directed to the color of a preferred- versus null-direction stimulus outside the RF, activity is enhanced by $3.8-15.3 \%$ (blue bars, $p=0.0012$, one sample $t$ - test). This effect is present in both monkeys individually ( $p=0.039$ with $n=15$ in monkey A, and $p=0.0062$ with $n=43$ for monkey B). There is no difference in the strength of the attentional effect between the color and direction task ( $p=0.44$, paired $t$ test). Moreover, the size of the two attentional effects is positively correlated across single neurons (illustrated by Supplementary Figure 4B), ruling out the possibility that some neurons show an attentional effect only in the direction, and others only in the color task.

Thus, the results in the color task demonstrate enhanced processing of irrelevant motion signals outside the spatial focus of attention. This is consistent with a recent report, where multi-unit activity in primary visual cortex was enhanced if distant segments of a relevant object overlapped with the RFs of the recording site ${ }^{12}$. Since spatial attention remains constant between our conditions, and is directed to a location distant from the RF of the recorded neurons, space-based modulations cannot account for the effects we observed. Instead, potential explanations must acknowledge some transfer of attention from color to direction of motion.

Where could such a transfer take place? On the one hand, there might be an object-based transfer of attention from color to direction of motion, which takes place at the spatial focus of attention (i.e., within the attended object ${ }^{13,14}$ ) and is followed by a global activation of the cor- 
responding motion-feature system (focal transfer). On the other hand, there might be a global modulation of the attended color outside the spatial focus of attention, which automatically transfers to the irrelevant motion feature in each object that has the attended color ${ }^{15}$. In the latter case, the transfer of attention would not only happen within objects at the spatial focus of attention (global transfer). Contrasting color-task conditions of identical versus differently colored stimuli we find single-unit evidence for both a focal and a global transfer of attention. Fig. 3 illustrates conditions, in which the animals attended either to the color or to the direction of the stimulus outside the RF. In the color task, if the attended stimulus moved in the preferred direction of the recorded neuron (A), neuronal responses did not differ between conditions of same vs. different colors (B, gray bars, $p=0.34$,one sample $t$ - test). This absence of a difference is consistent with a focal transfer of attention from color to motion, activating the preferred-direction feature system to an equal extent in both conditions. As expected, we obtained the same result in the direction task (B, white bars, $p=0.45$, one sample $t$ - test). In contrast, if the animals attended to the color of the stimulus outside the RF moving in the null direction of the recorded neuron (C), activity was higher if the stimulus inside the RF had the same color ( $\mathrm{D}$, gray bars, $p=0.0015$, one sample $t$ test). This increased activity is consistent with a global transfer of attention from color to motion, enhancing the processing of the remote motion signal only for stimuli having the attended color. Importantly, there is no difference between the corresponding stimulus conditions in the motion task, in which the animals did not attend to the color of the stimulus $(\mathrm{D}$, white bars, $p=0.49$, one sample $t$ - test). Thus, if the irrelevant feature of an attended object is motion in the preferred direction of the recorded neuron, a focal transfer of attention takes places and direction-specific 
feature-based attention dominates neuronal responses. In contrast, if the irrelevant feature of an attended object is motion in the null direction of the recorded MT neuron, an additional color-based global transfer can also become evident.

One might argue that the attentional modulations of MT neurons observed in the color task do not reflect a transfer of attention, but might rather be explained by attention divided between color and motion. More specifically, the animals might always attend to the direction of motion, even in the color task, and only at post-perceptual stages decide whether or not to respond. In this case, enhanced MT activity in the color task would simply reflect direction-specific feature-based attention effects, rather than any transfer of attention from color to motion. However, the data shown in Figure 3D allow us to reject this alternative account. The observed enhancement of MT activity for same vs. different colors in the color task (gray bars) cannot result from partial attention to the direction of motion because here the attended direction would be identical. Moreover, if the enhancement resulted from partial attention to color one would expect to find a similar enhancement in the direction task (white bars). Since there is no effect of same vs. different colors in the direction task, we can rule out any explanation based on divided attention.

While it is generally assumed that color and direction of motion are processed in anatomically distinct and functionally separate visual processing streams, several neurophysiological studies have shown that information about color is indeed available to the visual motion processing system ${ }^{16-21}$. Given such evidence, we want to emphasize that our findings do not reflect a featurebased effect of color. First, our measurements were conducted under neuronal isoluminance condi- 
tions by presenting pairs of yellow and blue RDPs that provided equally strong input for individual MT cells (see Methods provided as Supplementary Information). Second, even in the absence of isoluminance, any feature-based effect of color would cancel out since we always averaged across the two colors.

In summary, our data document neural correlates of object-based attention at the singleneuron level in extrastriate visual cortex. In macaque area MT, the processing of irrelevant motion signals is modulated if attention is directed to the color of the stimulus or even of another object moving elsewhere in the visual field. This provides evidence for a focal and a global transfer of attention between visual features. Assuming that such transfers apply to all stimulus features, these object-based attentional mechanisms would enhance responses to all those objects in a visual scene that share at least one feature with the currently attended object. This could account for the object-based attentional effects observed in psychophysical ${ }^{22-26}$ and functional brain imaging studies ${ }^{13,27}$ Our observations extend the feature-similarity model of attention ${ }^{10}$ to objects, in that attention creates an integrated saliency map, i.e. a representation of the visual scene in which the saliency of individual objects represents their multi-dimensional similarity to the currently attended object $^{28}$.

1. Posner, M. I. Orienting of attention. Quarterly Journal of Experimental Psychology 32, 3-25 (1980)

2. Sàenz, M., Buraĉas, G. T. \& Boynton, G. M. Global effects of feature-based attention in human visual cortex. Nature Neuroscience 5, 631-632 (2002). 
3. Duncan, J. Selective attention and the organization of visual information. Journal of Experimental Psychology: General 113, 501-517 (1984).

4. Desimone, R. \& Duncan, J. Neural mechanisms of selective visual attention. Annual Review of Neuroscience 18, 193-222 (1995).

5. Treue, S. Neural correlates of attention in primate visual cortex. Trends in Neurosciences $\mathbf{2 4 ,}$ 295-300 (2001).

6. Reynolds, J. H. \& Chelazzi, L. Attentional modulation of visual processing. Annual Review of Neuroscience 27, 611-647 (2004).

7. Maunsell, J. H. R. \& Treue, S. Feature-based attention in visual cortex. Trends in Neurosciences 29, 317-322 (2006). URL http://wWw.sciencedirect.com/science/article/B6T0V-4 JXRWXP-1/2/6239c59

8. Felleman, D. J. \& Van Essen, D. C. Distributed hierarchical processing in the primate cerebral cortex. Cerebral Cortex 1, 1-47 (1991).

9. Ungerleider, L. G. \& Mishkin, M. Two cortical visual systems. In Ingle, D. J., Goodale, M. A. \& Mansfield, R. J. W. (eds.) Analysis of Visual Behavior, 549-586 (MIT Press, Cambridge, MA, 1982).

10. Treue, S. \& Martínez-Trujillo, J. C. Feature-based attention influences motion processing gain in macaque visual cortex. Nature 399, 575-579 (1999). 
11. Martínez-Trujillo, J. C. \& Treue, S. Feature-based attention increases the selectivity of population responses in primate visual cortex. Current Biology 14, 744-751 (2004).

12. Roelfsema, P. R., Lamme, V. A. \& Spekreijse, H. Object-based attention in the primary visual cortex of the macaque monkey. Nature 395, 376-381 (1998).

13. O'Craven, K. M., Downing, P. E. \& Kanwisher, N. fMRI evidence for objects as the units of attentional selection. Nature 401, 584-587 (1999).

14. Sohn, W., Chong, S. C., Papathomas, T. V. \& Vidnyanszky, Z. Cross-feature spread of global attentional modulation in human area MT+. Neuroreport 16, 1389-1393 (2005).

15. Melcher, D., Papathomas, T. V. \& Vidnyanszky, Z. Implicit attentional selection of bound visual features. Neuron 46, 723-729 (2005).

16. Gegenfurtner, K. R. et al. Chromatic properties of neurons in macaque MT. Visual Neuroscience 11, 455-466 (1994).

17. Dobkins, K. R. \& Albright, T. D. What happens if it changes color when it moves?: the nature of chromatic input to macaque visual area MT. Journal of Neuroscience 14, 48544870 (1994).

18. Croner, L. J. \& Albright, T. D. Segmentation by color influences responses of motion-sensitive neurons in the cortical middle temporal visual area. Journal of Neuroscience 19, 3935-3951 (1999). 
19. Thiele, A., Dobkins, K. R. \& Albright, T. D. Neural correlates of chromatic motion perception. Neuron 32, 351-358 (2001).

20. Seidemann, E., Poirson, A. B., Wandell, B. A. \& Newsome, W. T. Color signals in area MT of the macaque monkey. Neuron 24, 911-917 (1999).

21. Nassi, J. J., Lyon, D. C. \& Callaway, E. M. The parvocellular LGN provides a robust disynaptic input to the visual motion area MT. Neuron 50, 319-327 (2006). URL http://wWw.sciencedirect.com/science/article/B6WSS-4JS15WY-K/2/893cf0b:

22. Valdés-Sosa, M., Cobo, A. \& Pinilla, T. Transparent motion and object-based attention. Cognition 66, B13-B23 (1998).

23. Blaser, E., Pylyshyn, Z. W. \& Holcombe, A. O. Tracking an object through feature space. Nature 408, 196-199 (2000).

24. Reynolds, J. H., Alborzian, S. \& Stoner, G. R. Exogenously cued attention triggers competitive selection of surfaces. Vision Research 43, 59-66 (2003).

25. Mitchell, J. F., Stoner, G. R., Fallah, M. \& Reynolds, J. H. Attentional selection of superimposed surfaces cannot be explained by modulation of the gain of color channels. Vision Research 43, 1323-1328 (2003).

26. Mitchell, J. F., Stoner, G. R. \& Reynolds, J. H. Object-based attention determines dominance in binocular rivalry. Nature 429, 410-413 (2004). 
27. Schoenfeld, M. A. et al. Dynamics of feature binding during object-selective attention. Proceedings of the National Academy of Sciences 100, 11806-11811 (2003).

28. Treue, S. Visual attention: the where, what, how and why of saliency. Current Opinion in Neurobiology 13, 428-432 (2003).

Supplementary Information is linked to the online version of the paper.

Acknowledgments This research is supported by the BMBF grant 01GQ0433.

Competing Interests Statement The authors declare that they have no competing financial interests.

Correspondence Correspondence and requests for materials should be addressed to S.T. (email: treue@gwdg.de). 
Figure 1 Selective attention task. Examples of the trial sequences for the direction task (upper sequence) and color task (lower sequence). After the monkeys fixated and depressed a lever, a small cue briefly appeared (500 ms), indicating the spatial location of the upcoming target stimulus and the attentional task to be performed. In the direction task, the cue was a small, achromatic moving RDP and instructed the monkey to respond to a change in direction of the cued stimulus (the target). In the color task, the cue was a stationary, colored RDP, instructing the monkey to respond to a change in the color of the target. After cue offset, two moving RDPs were presented at equal eccentricity in opposite visual hemifields, one of them inside the RF of the neuron under study. Changes in the stimuli could occur 500-3550 ms following cue offset. The red circle indicates the focus of attention. Examples of trial sequences where the target stimulus was the one on the left are not shown here. 
Figure 2 Effects of directing attention to different features of a colored motion stimulus outside the RF. (A) Attending to the motion signal of a preferred-direction (red dotted trace) versus null-direction stimulus (black dotted trace) outside the RF enhances activity of a representative MT neuron (feature-based attention). In line with object-based attention, the same modulation is evident if attention is directed to the color of these stimuli (solid traces). (B) Firing rates averaged over a population of $58 \mathrm{MT}$ neurons. (C) Distribution of the strength of attentional modulation for the recorded population, separately for conditions in which attention was directed to the motion signal (gray bars) or to the color (blue bars). Binning is based on the attentional index $A I=\left(f r_{\text {pref }}-f r_{\text {null }}\right) /\left(f r_{\text {pref }}+f r_{\text {null }}\right)$, where fr represents the firing rate in the corresponding attentional condition for the time window (300-800 ms relative to stimulus onset) marked by the black horizontal bar in Panel C. The top scale indicates the corresponding percentages. Both histograms are shifted to positive values, indicating that attention to color modulates processing of the irrelevant motion signal in MT, even outside the spatial focus of attention (the cross indicates the mean attentional index and the 95\%-confidence interval). 
Figure 3 Effects of directing attention to identical vs. differently colored stimuli outside the RF. (A) Attention is directed to the preferred-direction stimulus outside the RF. The color of the attended stimulus is either identical to or different from the color of the stimulus inside the RF. (B) Distribution of the strength of the modulation for the recorded population, separately for conditions in which attention was directed to the color (gray bars) or to the motion signal (white bars). Conventions as in Figure 2. Binning is based on the modulation index $M I=\left(f r_{s a m e}-f r_{d i f f}\right) /\left(f r_{\text {same }}+f r_{d i f f}\right)$, where $f r$ represents the firing rate in same color and different color conditions, respectively. Both histograms are centered on zero, indicating that similarity in terms of color has no effect if the preferred-direction stimulus outside the RF is attended. (C) Attention is directed to the null-direction stimulus outside the RF, with the color of the attended stimulus being either identical to or different from the stimulus inside the RF. (D) Distribution of the strength of the modulation for the recorded population, separately for conditions in which attention was directed to the color (gray bars) or to the motion signal (white bars). In the color task, the histogram is shifted to the right indicating that for the majority of the cells firing rates are higher if the colors of the stimuli are identical compared to when they are different. In the direction task, the corresponding histogram is centered on zero. Therefore, similarity in terms of color influences firing rates if the color, but not the direction, of the null-direction stimulus outside the RF is attended. 


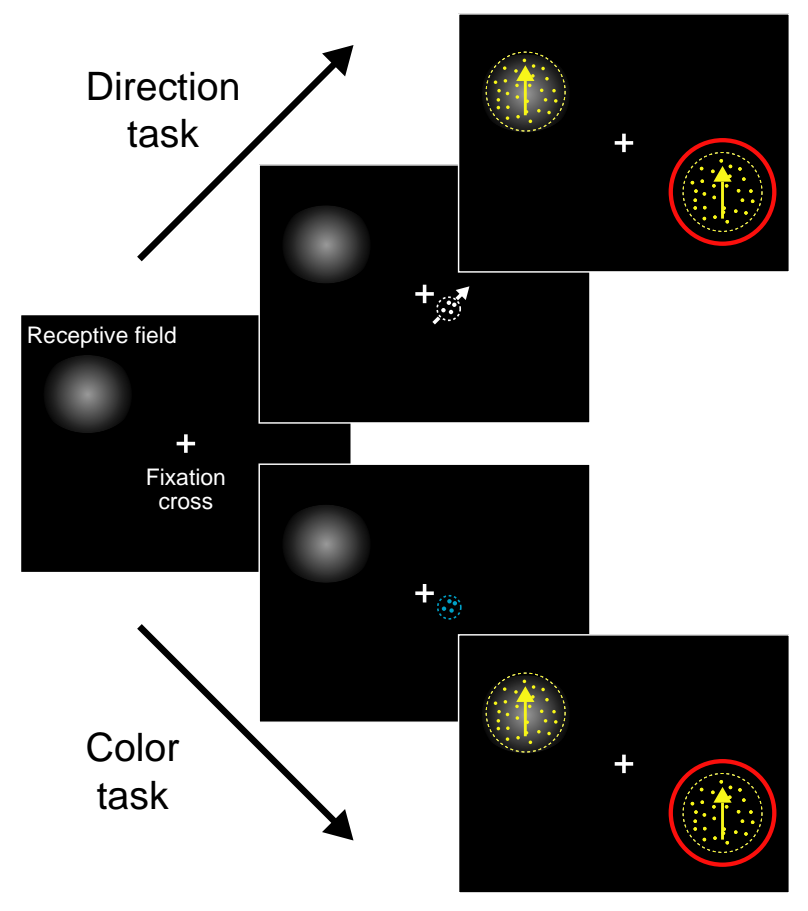

Figure 1 


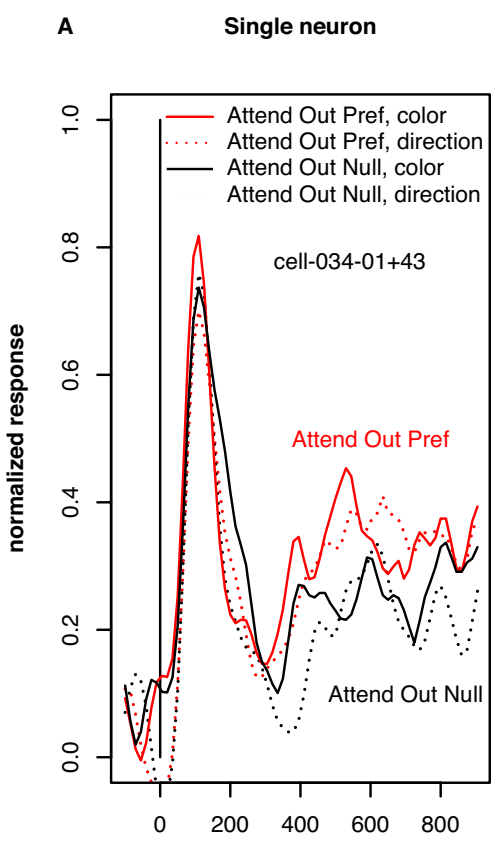

time from stimulus onset [ms]
B

Population responses

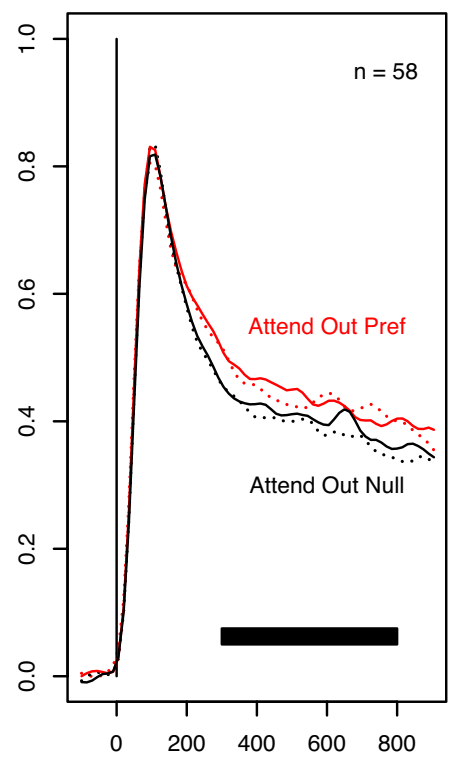

time from stimulus onset [ms]

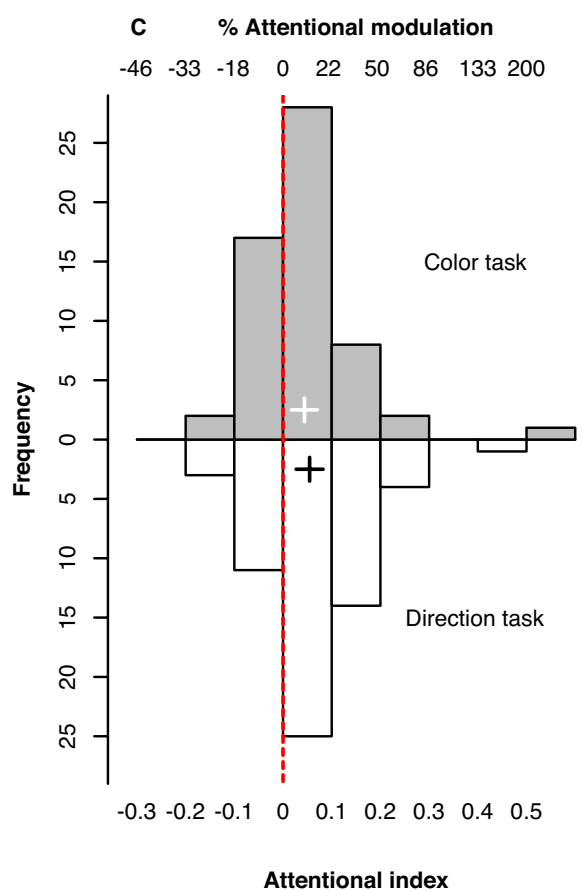

Attentional index

Figure 2 
A

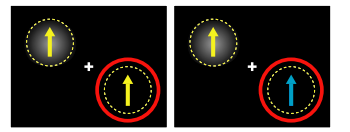

B

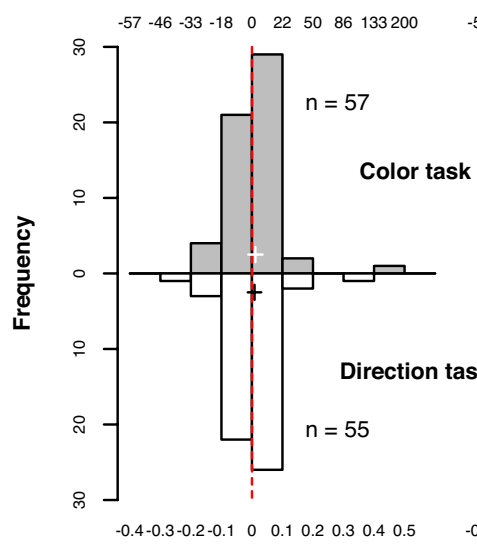

Modulation Index
C



(1)

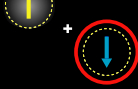

D \% modulation

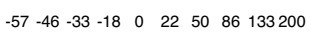



Modulation Index

Figure 3 


\title{
SUPPLEMENTARY ONLINE INFORMATION
}

\section{Object-based Attention Modulates Activity of Single Neurons in Primate Visual Cortex}

\author{
Steffen Katzner, Laura Busse, Stefan Treue
}

\section{Supplementary Figures and Legends}
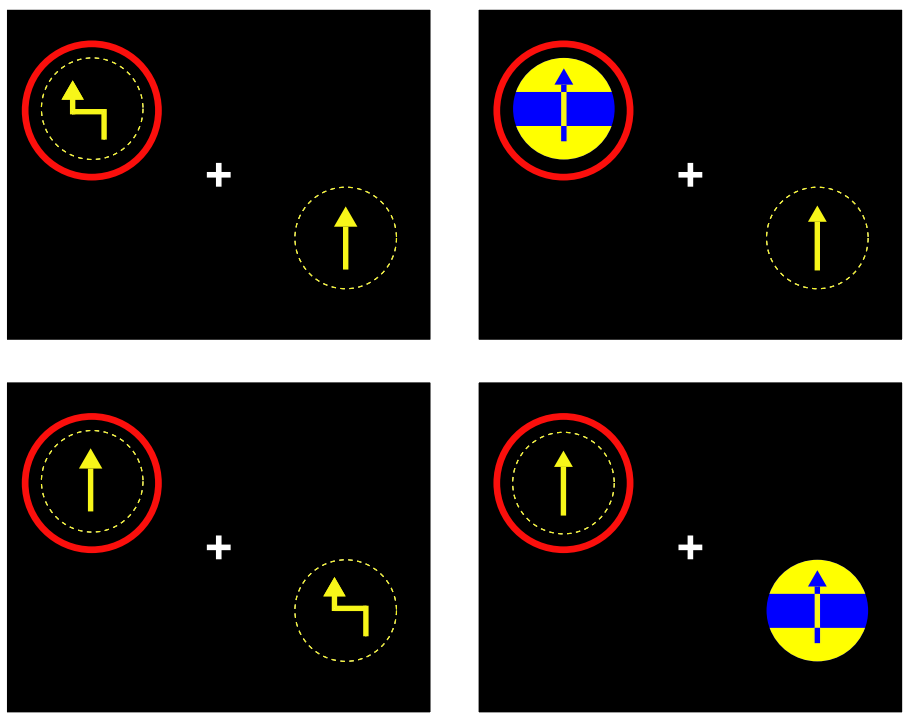

Supplementary Figure 1. Target event and three different distractor events in the direction task. The monkeys were only rewarded for responding to a change in the direction of motion of the cued stimulus (top left panel). Trials were terminated without reward if they responded to any of the following distractor types: a change in the color of the cued stimulus (top right, 'dimension distractor'), a change in the direction of motion of the uncued stimulus (bottom left, 'location distractor'), or a change in the color of the uncued stimulus (bottom right, 'dimension \& location distractor'). Trials were also terminated without reward if the monkeys missed the target or broke fixation. Corresponding events were used in the color task. 
A

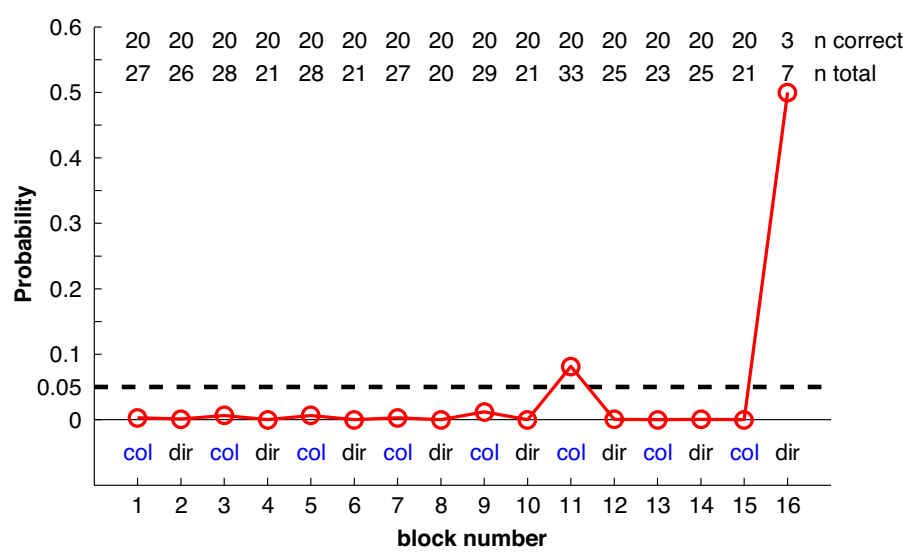

B

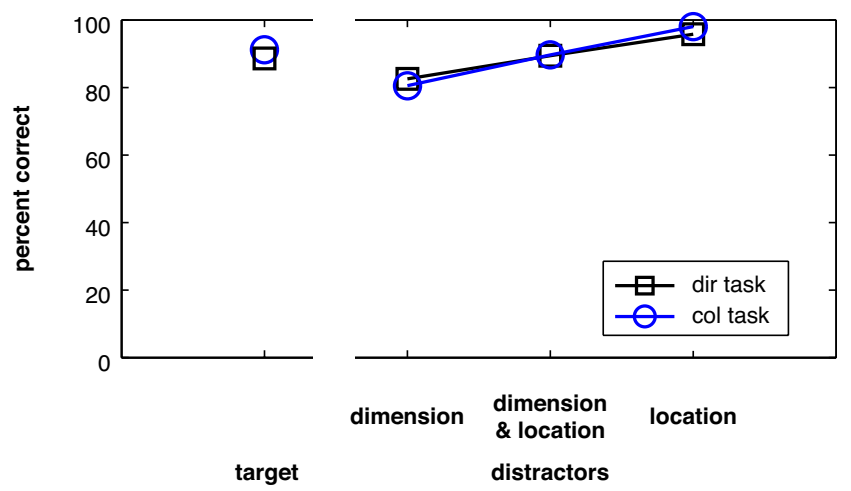

Supplementary Figure 2. Analyses of behavioral performance. (A) Blockwise analysis of overall behavioral performance. Representative recording session during which the monkey performed 16 blocks of color (col) and direction (dir) tasks, alternating every 20 correctly completed trials. Rows at the top indicate the number of correct trials and the total number of trials (excluding trials that were terminated because of fixation breaks), separately for every block. Depicted is, for every single block, the probability that 20 hits can be obtained purely by chance, given the total number of trials in that block, and assuming that the monkey was guessing (binomial test with probability $p$ of success $=0.5$ ). The dashed line marks the performance-based criterion for including individual blocks in all subsequent analyses. In this particular session, blocks 11 and 16 were excluded (in the last block the monkey only performed 7 trials, after which the recording session was ended). For the remaining 14 blocks, it is obvious that the monkey reliably performed alternating color and direction tasks. (B) Average performance for targets and for the different distractor types. Included are only blocks that meet the behavioral performance criterion. Performance for the targets (left part) was very high and the different distractor types (right part) had, on average, very little impact on performance. The pattern of results validates that the monkeys followed the attentional instructions and selectively attended to the cued feature of the cued stimulus. 

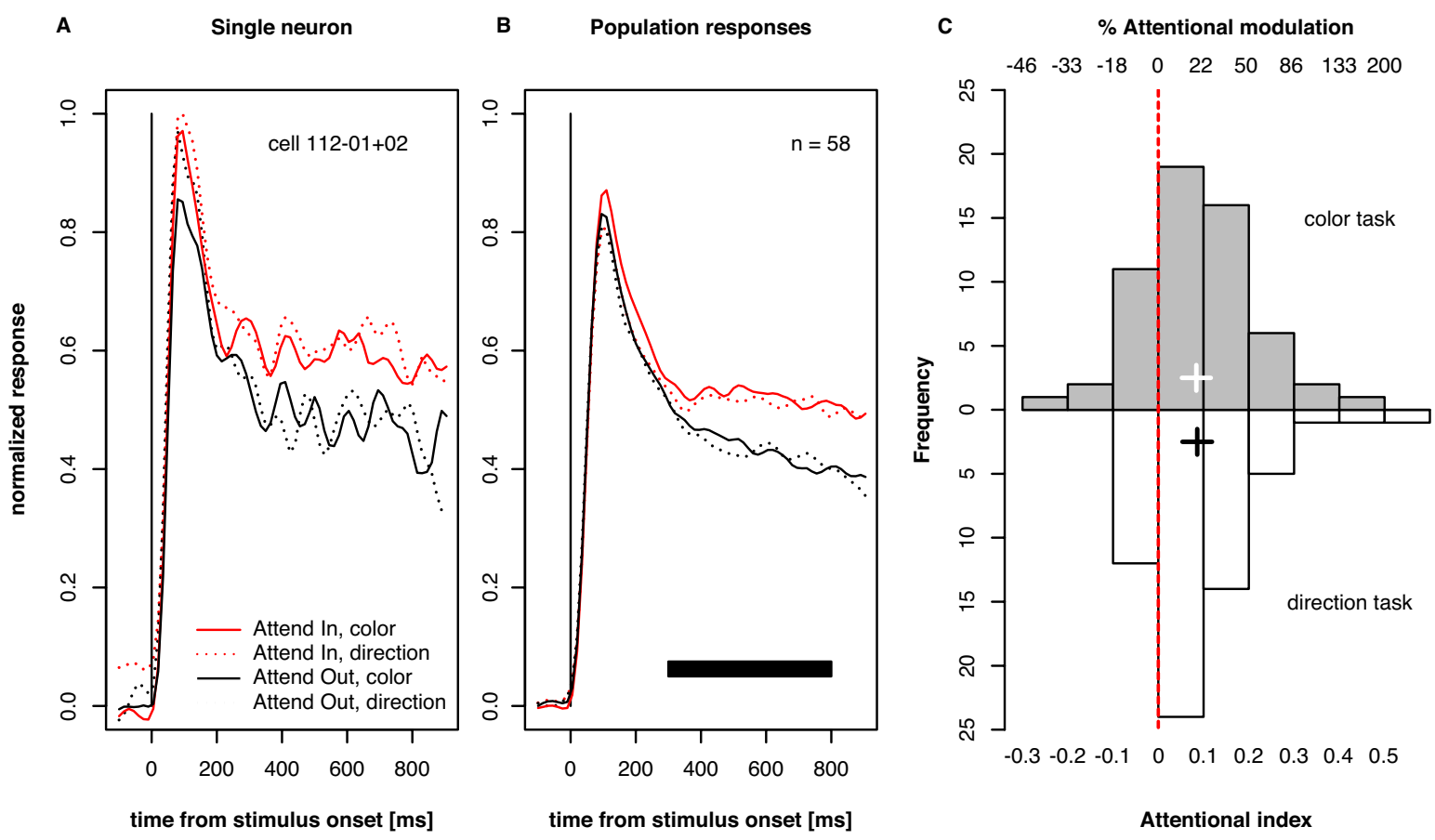

Supplementary Figure 3. Effects of directing attention to different features of a colored, moving stimulus. (A) Attending to the motion signal of a preferred-direction stimulus inside (red dotted trace) versus outside (black dotted trace) the RF enhances activity of a representative MT neuron. Remarkably, the same modulation is evident if attention is directed to the color of these stimuli (solid traces). (B) Firing rates averaged over a population of 58 MT neurons. (C) Distribution of the attentional effects for the recorded population, separately for conditions in which attention was directed to the motion signal (gray bars) or to the color (blue bars). Both histograms are shifted to positive values, indicating that attention modulates processing of the motion signal in MT, even if directed to the color of a moving stimulus. 

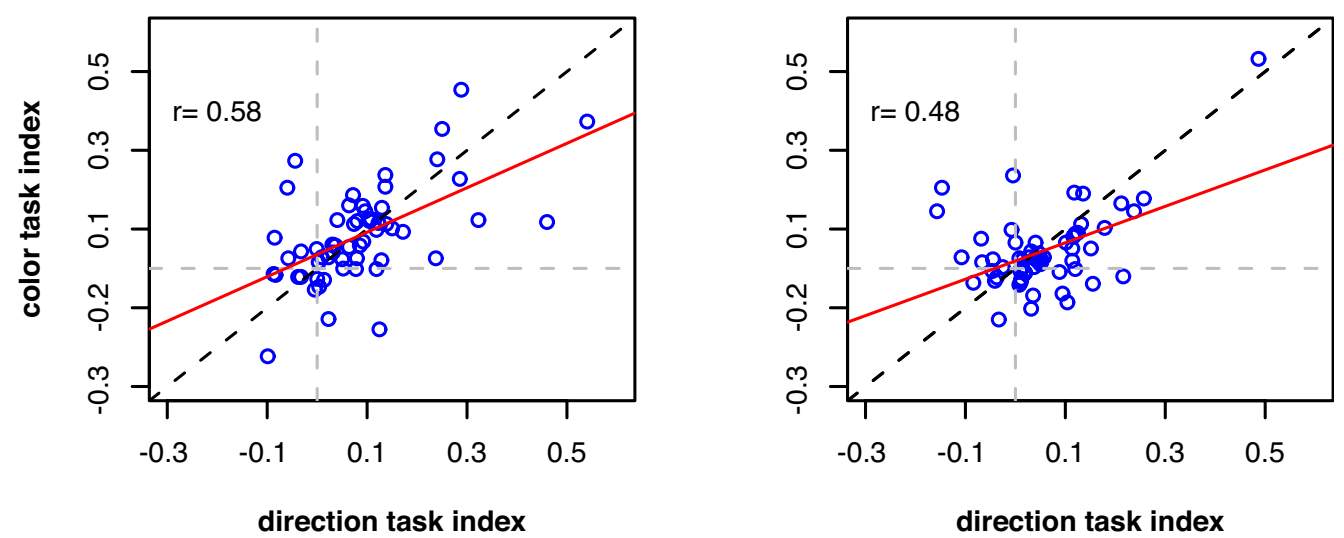

Supplementary Figure 4. Attentional effects in the direction task and in the color task are positively correlated. The scatter plots show attentional indices in the direction and the color task, in a condition where attention is directed to the preferred-direction stimulus inside versus outside the RF (A, data not shown in main text), or to a preferred- versus null-direction stimulus outside the $\mathrm{RF}$ (B, data from histogram in Figure 2C). Circles represent single neurons $(n=58)$. The bestfit least-squares lines are shown in red. An Analysis of Covariance (ANCOVA) confirmed that the intercept of the fitted line is not different from zero $(p=0.18)$, and that there is no difference in intercepts between A and $\mathrm{B}(p=0.42)$. Here, an intercept term different from zero would indicate systematic differences between the attentional indices in the direction task and color task. While the slope parameter significantly deviates from 1 ( 0.46 for $\mathrm{A}, p=0.00016)$, there is also no difference in slopes between the two conditions $(p=0.51)$. Overall, the ANCOVA confirms that there is a strong positive relationship between the magnitude of the attentional effects in the direction and in the color task for individual cells, and that this relationship holds true for both attentional comparisons.

\section{Supplementary Methods}

All experimental procedures were approved by the local animal research committee and complied with relevant laws and institutional guidelines. Headpost and recording chamber were implanted using standard surgical techniques ${ }^{11}$.

Behavioral task. Two monkeys (Macaca mulatta) were trained to attend either to the motion signal (direction task) or to the color (color task) of a moving random dot pattern (RDP) (Fig. 1, main text). To start a trial, the animal had to maintain fixation within a window of $1.25^{\circ}$ radius, centered on a $0.2^{\circ}$ fixation square. $150 \mathrm{~ms}$ after depressing a lever, a cue appeared for $500 \mathrm{~ms}$, signaling the position of the relevant stimulus (target location) and indicating whether a direction or a color task was required (target feature). After the cue was removed, two moving colored RDPs were presented at equal eccentricity in opposite visual hemifields, one of 
them inside the classical receptive field (RF) of the neuron under study. To ensure that the monkeys were correctly attending to the cued feature of the cued stimulus, one or two of the following events could happen within the next 500-3550 ms, randomized in time and order: the target feature appeared at the target location, the target feature appeared at the uncued location ('wrong location' distractor), the uncued feature appeared at the target location ('wrong feature' distractor), or the uncued feature appeared at the uncued location ('wrong location and feature' distractor) (Supplementary Figure 1). In the direction task, the monkeys received a liquid reward for releasing the lever within a response time window of 100-500 ms following a brief change in the direction of motion of the cued stimulus. In contrast, in the color task the monkeys were rewarded for responding to a brief change in the color of the cued stimulus. Trials were ended immediately following any response. If only distractor events occurred, the monkeys were required to continue depressing the lever and were rewarded at the end of the trial (4050 ms after cue offset). While distractor events at the wrong location allowed us to control that the monkeys attended to the target location, distractor events in the wrong feature guaranteed that they selectively attended to the target feature. Trials were terminated without reward if the monkeys responded to any of the distractor types, did not respond to the target feature at the target location, or broke fixation. Color and direction tasks were performed in separate blocks of trials, alternating every 20 correctly completed trials.

Apparatus and visual stimuli. Monkeys were seated in a primate chair with their head restrained at a distance of $57 \mathrm{~cm}$ from a computer monitor (resolution 40 pixels per degree of visual angle, refresh rate $76 \mathrm{~Hz}$ ). The eye position was monitored with a high-speed videobased eye tracker at a sampling rate of $230 \mathrm{~Hz}$ (ET49, Thomas Recording, Giessen, Germany). Our stimuli consisted of RDPs moving within a stationary virtual aperture. A single dot subtended $0.1^{\circ}$ of visual angle and the dot density was $8 \mathrm{dots} / \mathrm{deg}^{2}$. The size of the RDP, the speed of the dots, and the direction of motion were selected to match the preferences of the neuron under study. On a given trial, each RDP was independently assigned one of two neuronally isoluminant colors (blue versus yellow), and one of two motion directions (preferred versus null direction of the recorded neuron). In case of a direction change, all dots simultaneously changed their direction by $30-60^{\circ}$, depending on eccentricity and speed of the target stimulus. For color changes, $80 \%$ of all dots changed their color to either yellow or blue, depending on the original stimulus color. All changes lasted for $132 \mathrm{~ms}$ before the original stimulus properties were restored. The cues consisted of small RDPs subtending $0.75^{\circ}$ of visual angle, with a dot size of $0.075^{\circ}$ and a density of $40 \mathrm{dots} / \mathrm{deg}^{2}$. They were always presented at a distance of $2^{\circ}$ from fixation, positioned on a virtual line connecting the fixation point to the target location. For the direction cue, dots were achromatic and moved at a speed of $3^{\circ} / s$ in the direction which had to be detected. In the color task, the dots of the cue were stationary and were plotted in the to-be-detected color.

Neuronal recording and data collection. Single-unit activity was recorded from area MT using a five-channel multi-electrode recording system (Mini-matrix, Thomas Recording, Plexon data acquisition system). For most of the recording sessions, five electrodes were simultaneously advanced to isolate individual MT neurons with overlapping RFs. Cells were characterized as MT neurons based on directional tuning, receptive field location, and position in the cortex. The locations and sizes of individual RFs were mapped manually using a moving bar. Directional and speed tuning were determined by presenting a single RDP inside the joint RF, moving in 12 different directions for each of 8 different speeds $(0.5-64 \mathrm{deg} / \mathrm{s})$, while mon- 
keys were maintaining fixation. Preferred direction was estimated by fitting a Gaussian to the individual neuronal responses, plotted as a function of stimulus direction, separately for each speed. Out of the 58 recorded neurons, 23 were obtained from parallel recordings (either from two, three, or four cells simultaneously). For these groups of cells, the stimulus for the subsequent experiment was optimized for the neuron exhibiting the strongest direction selectivity. Neuronal isoluminance was established by presenting a preferred-direction RDP inside the receptive field (3.7-5.7 s) that changed its color every $500 \mathrm{~ms}$, while the monkey held fixation. Colors were randomly selected from a sample consisting of a single blue of fixed intensity $\left(45 \mathrm{~cd} / \mathrm{m}^{2}\right)$ and various intensities of yellow, bracketing the level of intensity for blue (31-63 $\mathrm{cd} / \mathrm{m}^{2}$ ). Selecting the appropriate level of intensity for yellow ensured that both colors provided equally strong inputs for individual MT neurons.

Analysis of behavioral data. The comparison between neuronal activity between the direction and color task critically requires that the monkeys were reliably following the attentional instructions given by the cue. To ensure this, we examined the monkeys' behavioral performance in every single block of trials for each recording session. Specifically, we calculated the probability that the number of hits within a block could be obtained by chance, given the total number of trials in that block, and assuming that the monkey was guessing as to whether he should be responding to a color or a direction change (binomial test with probability $p$ of success $=0.5)$ (Supplementary Figure 2A). For all further analyses, we included only those individual blocks of trials, for which the behavioral performance was reliably different from chance level $(p<0.05)$. Subsequently, an Analysis of variance (ANOVA) was performed, separately for targets and distractors, to test for systematic dependencies of performance on the two attentional tasks (Supplementary Figure 2B). Target-related performance was assessed with a one-way ANOVA involving the within-subjects factor type of task (direction task vs. color task). Both monkeys successfully detected most of the targets, with a slightly better performance in the color task $(91.08 \%)$ than in the direction task $(88.61 \%)$. Distractor-related performance was examined with a two-way ANOVA involving the within-subjects factors type of task and type of distractor ('wrong location' versus 'wrong dimension' versus 'wrong location \& dimension'). On average, both monkeys successfully ignored most of the distractors $(89.32 \%)$, with better performance for location distractors $(96.89 \%)$ than for dimension \& location distractors (89.54\%), and for dimension distractors (81.53\%). Most important, performance differences between the different distractor types were identical for the color and direction task ( $p=0.1$, Greenhouse-Geyser corrected).

Analysis of neuronal data. For all cells included in the analysis of neuronal data, responses to the preferred direction were at least three times as large as responses to the null direction. For any given recording session, only those blocks of trials were included for which the analysis of behavior ensured that the monkeys were following the attentional instructions (Supplementary Figure 2). Finally, individual trials were only included if they were correctly completed, and for these trials neuronal data were only analyzed until the first change in either of the two stimuli occurred. To obtain reliable estimates of single neuron average firing rates, a number of correct repetitions were collected for each neuron in all comparisons (Figure 2: minimum $=5$, mean $=20.1$, sd $=7.2$; Figure 3: minimum $=3$, mean $=12.5$, sd $=4.75$; Supplementary Figure 3: minimum $=7$, mean $=23.4, \mathrm{sd}=8.8$ ). All firing rates plotted represent values of the spike density function at steps of $15 \mathrm{~ms}$, which were obtained by convolving spike trains with a Gaussian kernel ( $\sigma=30 \mathrm{~ms}$ ). For the main comparisons attentional effects were quantified 
by computing an attentional index, defined as the difference in firing rates between two conditions, divided by their sum, after subtraction of spontaneous firing rate. For plotting single neuron data and population activity, neuronal responses were normalized to the peak amplitude evoked by an unattended preferred-direction stimulus presented inside the RF. To assess statistical significance of the modulatory effects presented in Fig. 2, Fig. 3, and Supplementary Figure 3 one-sample or paired t-test (always two-tailed) were performed on the indices described in the respective figure captions. All of the comparisons also reached significance if the corresponding non-parametric Wilcoxon-test was used instead.

\section{Supplementary Notes}

This research is supported by the BMBF grant 01GQ0433. 


\subsection{Feature-based attentional integration of color and visual motion}

Functional specialization is one of the hallmarks of the visual system (Felleman and Van Essen, 1991). In the macaque brain, more than 30 visual areas have been identified so far, which can be broadly grouped into two major processing streams (Ungerleider and Mishkin, 1982). While the temporal pathway, including areas V1, V2, V4, and IT/TEO, is mainly concerned with processing object attributes like color, shape, and identity, the dorsal pathways, projecting from V1 to areas MT/MST and into the parietal cortex, contains neurons selective for motion information, position, and spatial relations. Although this classification scheme has been very influential in the study of the visual system, the separation between the two streams is far from complete.

In this experiment, we investigated whether and how color and motion information, processed in the ventral and dorsal stream, respectively, are integrated in a divided attention task. We find that RTs to targets consisting of both a color and direction change are too fast to be accounted for by separate and independent processing of the two features. Since this effect persists for target changes occurring on separate surfaces and across spatial positions we conclude that a feature-based attentional mechanism can best explain the results. 


\section{Feature-based attentional integration of color and visual motion}

\section{Steffen Katzner}

\section{Laura Busse}

\section{Stefan Treue}

\author{
Cognitive Neuroscience Laboratory, German Primate Center, \\ Göttingen, Germany

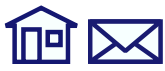 \\ Cognitive Neuroscience Laboratory, German Primate Center, \\ Göttingen, Germany

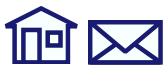 \\ Cognitive Neuroscience Laboratory, German Primate Center, \\ Göttingen, Germany
}

In four variants of a speeded target detection task, we investigated the processing of color and motion signals in the human visual system. Participants were required to attend to both a particular color and direction of motion in moving random dot patterns (RDPs) and to report the appearance of the designated targets. Throughout, reaction times (RTs) to simultaneous presentations of color and direction targets were too fast to be reconciled with models proposing separate and independent processing of such stimulus dimensions. Thus, the data provide behavioral evidence for an integration of color and motion signals. This integration occurred even across superimposed surfaces in a transparent motion stimulus and also across spatial locations, arguing against object- and location-based accounts of attentional selection in such a task. Overall, the pattern of results can be best explained by feature-based mechanisms of visual attention.

Keywords: reaction time, race model, attention, color, motion, transparent motion

\section{Introduction}

Functional specialization is one of the hallmarks of the primate visual cortex. Different attributes of a visual stimulus, like motion, depth, form, and color, are known to be processed in separate areas or even pathways of the visual cortex (Felleman \& Van Essen, 1991). A number of such specialized cortical areas have been identified, and they seem to form at least two processing streams (Ungerleider $\&$ Mishkin, 1982). The ventral pathway (mainly involving areas V1, V2, V4, TEO, and IT) shows specialization for the processing of color and shape. In contrast, areas in the dorsal pathway (V1, V2, V3, MT/MST) analyze information about motion and spatial relations. This notion of spatially separate and functionally independent, parallel processing streams represents an important conceptualization of visual information processing. However, the functional separation is far from complete (Ferrera, Nealey, \& Maunsell, 1992), and a large number of anatomical connections between these two pathways have been demonstrated, providing a neural substrate for interactions.

In reaction time $(\mathrm{RT})$ research, the combined processing of separable sensory signals has been studied with the redundant-target paradigm (Miller, 1982, 1986; Mordkoff \& Yantis, 1993). In a typical experimental situation, two different sensory signals are defined as targets and participants are required to make speeded responses if either of the two targets is detected. Of special interest is a condition in which both targets are presented simultaneously (redundant-target trials). This condition is then compared with those in which either of the targets is presented alone (single-target trials). It is typically found that RTs to redundant targets are faster than RTs to single targets, and this finding is commonly referred to as redundancy gain (Giray \& Ulrich, 1993; Iacoboni \& Zaidel, 2003; Krummenacher, Müller, \& Heller, 2001, 2002; Miller, 1982, 1986, 2004; Miller, Ulrich, \& Lamarre, 2001; Miniussi, Girelli, \& Marzi, 1998; Mordkoff, Miller, \& Roch, 1996; Mordkoff \& Yantis, 1991, 1993; Turatto, Mazza, Savazzi, \& Marzi, 2004).

Essentially, two classes of models have been advanced to explain this redundancy gain. The first class consists of race models. They are based on the idea that the two concurrent sensory signals are processed separately and independently and that responses can be initiated as soon as one of the two signals is detected. Sensory information for the two signals is not combined to initiate a response. Responses to redundant targets are particularly fast because they are produced by the faster of the two detection processes. The term race model illustrates the fact that responses are thought to be initiated by the winner of a race between the two separate detection processes. If one assumes that processing time randomly varies from trial to trial, and that the distributions of processing times for the two signals overlap, it follows that, on average, the time needed by the winner will be less than the time needed by either racer. For this reason, race models have also been characterized as producing statistical facilitation 
(Raab, 1962). The second class of models consists of coactivation models (Miller, 1982). In contrast to race models, sensory information for the two signals is combined to reach some decision criterion based on which responses are initiated. Here, responses to redundant targets are particularly fast because such a criterion will be reached faster if two processes combine in satisfying it. The term coactivation reflects the fact that two processes combine in activating a response.

\section{The race-model inequality}

Miller (1982) has provided a formal test to decide between these two classes of models with experimental RT data. In brief, he showed that all race models have to fulfill the following inequality:

$P\left(\mathrm{RT}<t \mid S_{1}\right.$ and $\left.S_{2}\right) \leq P\left(\mathrm{RT}<t \mid S_{1}\right)+P\left(\mathrm{RT}<t \mid S_{2}\right)$,

where $t$ is the time needed to respond to a signal and $S_{1}$ and $S_{2}$ are the two targets. Intuitively, this inequality formalizes an implicit constraint applying to all variants of race models: Responses to redundant targets $\left(S_{1}\right.$ and $S_{2}$, presented together) cannot be faster than the fastest response to either of the single targets ( $S_{1}$ or $S_{2}$, presented alone) of the single targets. Note that the three terms in this inequality represent the cumulative probability density function (CDF) of RT on redundant- and single-target trials, respectively. The CDFs obtained in a speeded detection task just need to be evaluated at the different values of $t$ to decide between race and coactivation models: If the inequality is violated for any of them, all variants of race models can be rejected.

In this study, we apply this logic to the processing of color and motion signals. We consider performance that is consistent with predictions of race models as evidence for separate and independent processing of these two stimulus dimensions. Conversely, performance inconsistent with predictions of race models would argue against separate and independent processing and rather support crossdimensional integration of color and motion signals.

In Experiment 1, we establish that redundancy gains can be observed for the stimulus dimensions color and direction of motion. We further demonstrate that explanations based on race models can safely be rejected, favoring crossdimensional integration of color and motion signals in speeded target detection tasks. In three subsequent experiments, we investigate in more detail whether the observed integration depends on specific stimulus attributes or task demands. Throughout, we find robust redundancy gains, as well as strong evidence for cross-dimensional processing of color and motion signals.

\section{Experiments}

\section{Experiment 1}

The purpose of Experiment 1 was to investigate whether redundancy gains can be observed for targets defined by a change in color or in the direction of motion. We used moving random dot patterns (RDPs) in a go/no-go target detection task, requiring speeded responses if a particular color (single color target), a particular direction of motion (single direction target), or both (redundant target) were presented and no response if the stimulus did not contain any of the designated targets.

\section{Methods}

The stimulus (Figure 1a) was composed of dots moving within a virtual circular aperture with a diameter of $5 \mathrm{deg}$. The dot density was 2 dots $/ \mathrm{deg}^{2}$ of visual angle. Each dot subtended 0.1 deg of visual angle. The RDP was plotted against a black background and centered on a yellow fixation point that was presented in the middle of the screen. Each trial started with an RDP that rotated around the fixation point at an angular speed of $2.9 \mathrm{deg} / \mathrm{s}$; all the dots were gray. In case of a color change, the dots changed to red, green, or blue. The four colors were equiluminant $\left(25 \mathrm{~cd} / \mathrm{m}^{2}\right)$. In case of a direction change, $70 \%$ of all dots started to translate into one of three linear motion directions: either upward, to the left, or to the right. The remaining $30 \%$ of the dots moved in random directions. This was done to prevent participants from solving the task by tracking a single dot. The stimulus was presented on a VGA monitor (Lacie, Electron22 Blue IV) operating at a refresh rate of $85 \mathrm{~Hz}$ and a resolution of 80 pixels/deg of visual angle. Presentation of the stimulus and recording of the responses were controlled by an Apple Power Mac G4 computer.

At the beginning of an experiment, participants were told which color and direction of motion were defined as targets. They were instructed to respond with a keypress on a computer keyboard (" $\mathrm{H}$ ") if the target color, the target
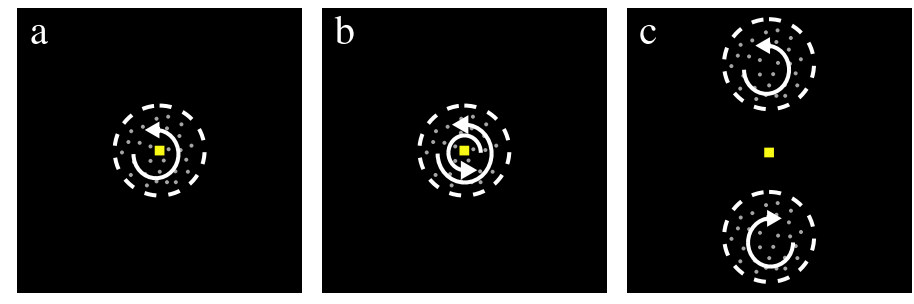

Figure 1. Stimuli used in Experiments 1-4. (a) Experiment 1. A single RDP rotating around the fixation square. (b) Experiment 2. Two superimposed RDPs rotating against each other. (c) Experiments 3 and 4 . Two rotating RDPs centered 3.75 and 7.5 deg above and below fixation in Experiments 3 and 4, respectively. 


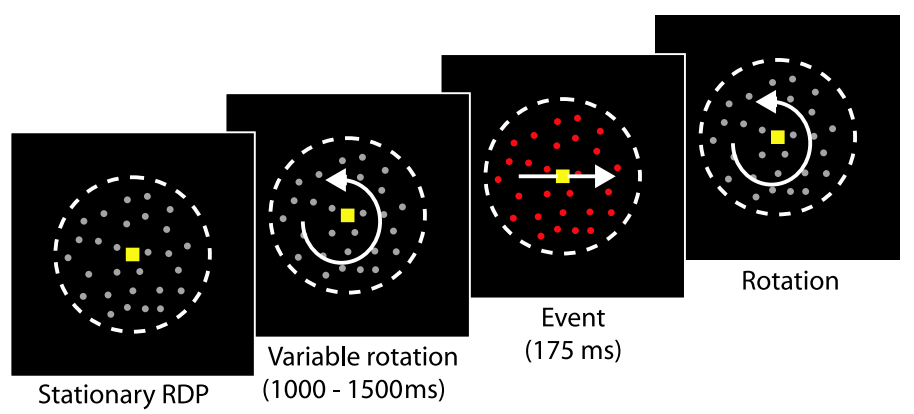

Figure 2. Trial sequence. A stationary pattern of gray dots was present at the beginning of each trial. It then rotated around the fixation square for 1,000-1,500 ms before changing its color, direction of motion, or both. After another period of $175 \mathrm{~ms}$, the RDP changed back to its initial rotation and color.

direction, or both were presented (go trials) and to withhold their response if no target was presented (no-go trials). The participants were told to respond as quickly as possible without making too many errors. The trial sequence is illustrated in Figure 2. A stationary dot pattern was presented, together with the fixation point, until participants triggered the beginning of a trial by pressing the space bar on the keyboard. Following this, the RDP rotated around the fixation point for $1,000-1,500 \mathrm{~ms}$. Then, one of the following events occurred: a change in color, a change in direction of motion, or both. The changes lasted for $175 \mathrm{~ms}$ before the original stimulus properties were restored. Following the participants' response, the stimulus was removed. If no response had been given within 1,000 ms after onset of the event, the trial was terminated and the response was considered a no-go. Auditory feedback was given at the end of each trial. The participants triggered the beginning of the next trial when they were ready to proceed.

Any redundancy gain would result in shorter RTs to redundant targets as compared to single targets. This, however, would be a comparison between a condition with two sensory events (redundant-target trials, with simultaneous changes in direction of motion and color) against a condition with only a single sensory event (single-target trials, with changes either in direction of motion or in color). Because two sensory events represent a much stronger signal compared with a single sensory event, this difference by itself could speed RTs in the redundant-target condition.
To make sure that potential redundancy gains do not result from a difference in the number of sensory events per se, we introduced single-target control conditions having two sensory events. In these control conditions, the single targets were combined with neutral events in the other stimulus dimension (i.e., a single color target was combined with a neutral change in direction, and a single direction target was combined with a neutral change in color). A neutral event is "neutral" in the sense that it appeared equally often in combination with go signals as well as with no-go signals. Consequently, the appearance of a neutral event was uninformative as to whether the participant should respond, whereas the appearance of a no-go event always signaled to withhold the response. Experiment 1 was divided into six blocks of 100 trials each. In a single block, there were 10 signal conditions (5 go and 5 no-go conditions, listed in that order): redundant targets, single color targets, single direction targets (the latter two will be referred to as "single target alone"), single color targets combined with neutral direction changes, single direction targets combined with neutral color changes ("single target + neutral"), redundant no-gos, single color no-gos, single direction no-gos, single color nogos combined with neutral direction changes, and single direction no-gos combined with neutral color changes. Overall, there were $50 \%$ go and $50 \%$ no-go trials. The 10 signal conditions were presented in a pseudorandomized order until 10 correct responses had occurred in each condition. Within each condition, the initial rotation of the RDP was clockwise for five trials and counterclockwise for the remaining five trials. Between blocks, participants were given a break of $5 \mathrm{~min}$. The assignment of three particular colors to go, no-go, and neutral conditions was counterbalanced across participants and remained unchanged throughout the entire experiment. To achieve the same level of difficulty for the go and no-go motion tasks, we always treated upward motion as the neutral direction for all participants. For half of the participants, rightward motion represented the go direction, whereas leftward motion represented the no-go direction; for the other half, this assignment was reversed. Table 1 summarizes one such combination of go, no-go, and neutral events forming the 10 signal conditions.

\section{Participants}

Each participant was tested in two sessions that were performed on separate days. A single session was divided

\begin{tabular}{|c|c|c|c|c|c|c|c|}
\hline & \multirow[b]{2}{*}{ Go signals } & \multirow[b]{2}{*}{ No-go signals } & & \multicolumn{2}{|c|}{ Go signals } & \multicolumn{2}{|c|}{ No-go signals } \\
\hline & & & & Color & Direction & Color & Direction \\
\hline Redundant & Red + right & Green + left & $\begin{array}{l}\text { Single alone } \\
\text { Single }+ \text { neutral }\end{array}$ & $\begin{array}{l}\text { Red } \\
\text { Red + up }\end{array}$ & $\begin{array}{l}\text { Right } \\
\text { Right + blue }\end{array}$ & $\begin{array}{l}\text { Green } \\
\text { Green + up }\end{array}$ & $\begin{array}{l}\text { Left } \\
\text { Left }+ \text { blue }\end{array}$ \\
\hline
\end{tabular}

Table 1. Experiment 1. Combination of go, no-go, and neutral events for participants instructed to detect rightward motion (direction target) or the color red (color target). The color green and leftward motion did not require a response. In the "single + neutral" condition, single color signals were accompanied by neutral direction changes (upward motion), whereas single direction signals were accompanied by neutral color changes (blue). 
into three blocks of approximately $10 \mathrm{~min}$ each. Each participant was instructed to respond to a particular color and a particular direction of motion at the beginning of the experiment. During the first session, participants completed a practice block to become familiar with the task. These practice blocks were not included in the analyses. Twelve participants (four men and eight women; ages 22-36, $M=27.1$, $S D=5.3$ ) were tested in Experiment 1 and were paid for their participation. All had normal or corrected-to-normal vision. They gave informed written consent and were naive as to the purpose of the experiment. All sessions were conducted in a dimly illuminated, quiet testing booth. Participants were comfortably seated with their head resting in a chin and forehead rest at a distance of $114 \mathrm{~cm}$ from the computer monitor.

\section{Data analyses}

Two analyses were performed. The first analysis addressed the redundancy gain and examined whether RTs to redundant targets were faster than RTs to single targets. Mean RTs were determined for each combination of participant and signal condition (redundant target, single color alone, single direction alone, single color + neutral direction, single direction + neutral color). For each participant, we selected the faster of the two mean RTs from the "single target alone" conditions ("fastest single alone"). Analogously, we determined the faster of the two mean RTs in the "single target + neutral" condition ("fastest single + neutral"; see Miller, 1982). Statistical significance was evaluated with a one-way ANOVA involving the withinsubjects factor of target type (redundant target vs. fastest single alone vs. fastest single + neutral).

The second analysis compared RT distributions between conditions to test for violations of the race-model inequality. Following Miller (1982), we compared the sum of the CDFs for the single-target conditions to the CDF for the redundant-target condition. First, we determined the CDFs for each combination of participant, block, and signal condition (redundant target, single color alone, single direction alone, single color + neutral direction, single direction + neutral color) by rank ordering the 10 RTs, which then represent an estimate of the unknown, true $\mathrm{CDF}$ at 10 percentiles (.05-.95). We then computed the sum of the CDFs in the "single target alone" condition ("sum of single alone") and, analogously, the sum of the CDFs in the "single target + neutral" condition ("sum of single + neutral"). The obtained CDFs were then averaged across blocks for a given participant and finally across participants. To test for violations of the race-model inequality, we compared the CDF for the redundant-target condition to the sum of the CDFs for the "single target alone" condition. Analogously, we compared the CDF for the redundant-target condition to the sum of the CDFs for the "single target + neutral" condition. The race-model inequality would be violated if RTs from the redundant-target CDF were faster than corresponding RTs from the summed CDF at any of the percentiles. Statistical significance was evaluated by conducting paired $t$ tests across participants at each of the 10 percentile pairs (redundant vs. "sum of single").

\section{Results and discussion}

\section{Redundancy gain}

Across participants, performance reached $89 \%$ correct for the no-go trials and varied between $99 \%$ and $100 \%$ correct for the five go conditions. Mean RTs across participants are shown in Figure 3. The ANOVA revealed a highly significant difference between mean RTs, $F(2,22)=33.73$, $M S E=126.39, p<.001$, Greenhouse-Geyser corrected. Post hoc analyses confirmed that RTs for redundant targets (328 ms) were faster than RTs for both "fastest single alone" (358 ms, $p<.001)$ and "fastest single + neutral" (363 ms, $p<.001)$. However, RTs for "fastest single alone" were not different from RTs for "fastest single + neutral" $(p=.28)$. A 95\% confidence interval for the main effect of target type indicates that redundancy gains are in the range of 26-40 ms under the present conditions.

\section{Race-model inequality}

Mean CDFs across participants are shown in Figure 4. Comparing the redundant-target $\mathrm{CDF}$ (pentagrams) to the sum of single alone CDF (circles) reveals a clear violation of the race-model inequality because the redundant-target CDF lies above and to the left of the "sum of single alone" CDF (upper panel in Figure 4).

Paired $t$ tests across participants at each of the 10 percentile points confirmed that RTs from the redundant signal CDF were reliably faster than RTs from the "sum of

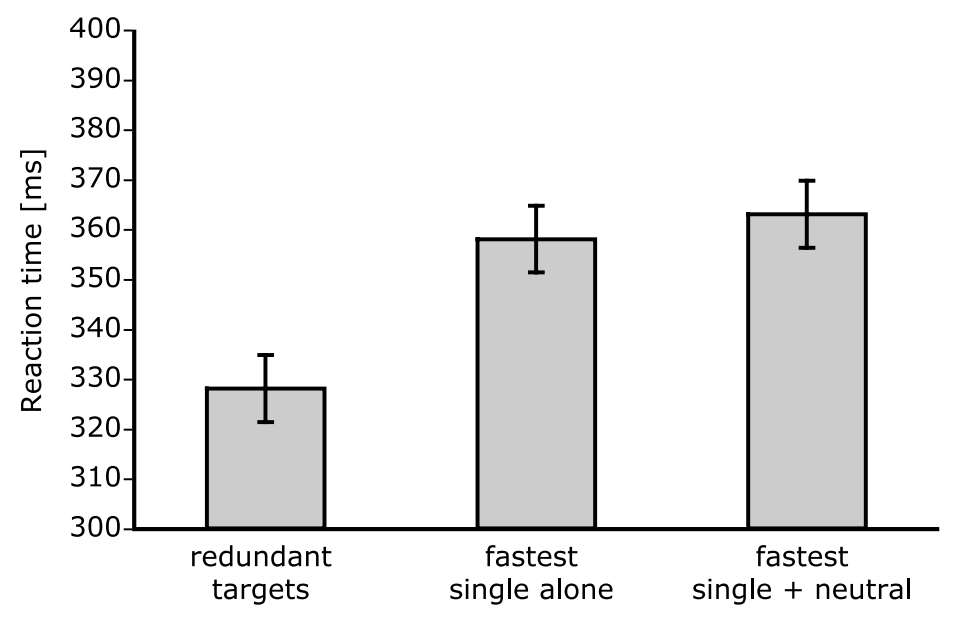

Figure 3. Experiment 1. RTs as a function of target type (redundant target vs. fastest single alone vs. fastest single + neutral). RTs to redundant targets are lower than RTs in the other two conditions ( $p<.001$ for both comparisons). There is no statistical difference between "fastest single alone" and "fastest single + neutral." Error bars indicate the 95\% confidence interval for the main effect of target type (see Loftus \& Masson, 1994). 

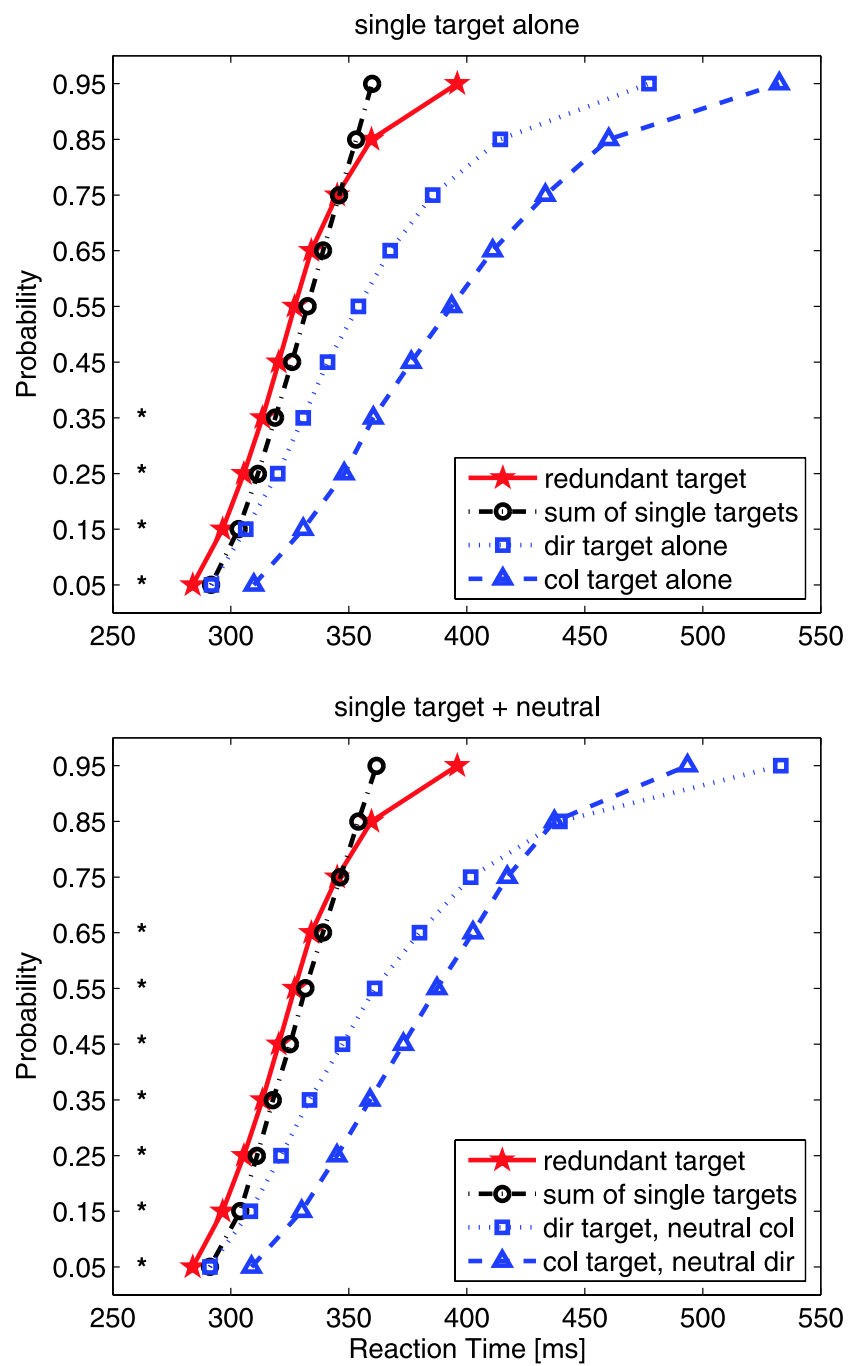

Figure 4. Experiment 1. Violations of the race-model inequality are revealed by comparing the redundant-target CDF (pentagrams) with the sum of the single-target CDFs (circles). Inconsistent with predictions of all race models, the redundant-target CDF lies above and to the left of the CDF for the sum of the single targets. This is the case for both "sum of single alone" (upper panel) and "sum of single + neutral" (lower panel). It shows that the fastest response to redundant targets can be faster than the fastest responses to single targets. Significance markers along the $Y$-axis indicate the percentiles at which RTs to redundant targets were reliably faster than corresponding RTs for the sum of the single CDFs.

single alone" $\mathrm{CDF}$ at the 5th, 15th, 25th, and 35th percentiles $(.001<p<.02)$. This shows that the fastest responses to redundant targets can in fact be faster than the fastest response to single targets, which is inconsistent with predictions of all race models. Analogously, we compared the redundant-target CDF to the sum of the CDFs in the "single target + neutral" condition to test for a violation of the race-model inequality when single targets are combined with neutral events (lower panel in Figure 4). As is apparent from this figure, the race-model inequality was again considerably violated. RTs were reliably faster for the redundant-target $\mathrm{CDF}$ at percentiles $0.05,0.15$, $0.25,0.35,0.45,0.55$, and $0.65(.001<p<.005)$.

The results argue against separate and independent processing of color and motion signals in a speeded target detection task with moving RDPs. We interpret this finding as evidence for cross-dimensional integration of color and motion information. In Experiment 1, neither the examination of redundancy gains nor the test for violations of the race-model inequality revealed any difference depending on whether we used single signals alone or single signals combined with neutral events. This finding is inconsistent with the idea that redundancy gains in the "single target + neutral" condition are simply due to response interference or cognitive inhibition effects. For instance, one could conceive that participants internally define the stimuli such that the designated color and direction were labeled as "go signals" and all others were considered "nogos." Resolving this conflicting information might have slowed down responses in the "single target + neutral" condition, thereby artificially producing a redundant-target effect (RTE), because redundant targets never contained conflicting information. However, because there is no difference between responses to single targets and single targets combined with neutral events (Figure 3), we can safely reject this possibility. Having demonstrated that adding a neutral signal to a single target has no inhibitory effect, we did not include the "single target alone" condition in all the succeeding experiments (i.e., we only used single targets that were combined with neutral events).

\section{Experiment 2}

The purpose of Experiment 2 was to investigate whether the observed cross-dimensional integration of color and motion signals requires that the two target signals belong to the same object. We rotated two populations of dots against each other, resulting in the percept of two superimposed surfaces (i.e., objects). This enabled us to present redundant targets always at the same location but either on the same surface or on different surfaces. If color and motion signals are integrated only if they belong to the same object, then we should observe violations of the race-model inequality only if redundant targets are presented on the same surface but not if they are presented on different surfaces.

\section{Methods}

The basic go/no-go target detection paradigm was identical to Experiment 1 except for the following changes. First, two RDPs were rotated against each other (Figure 1b). Second, because of superimposing two RDPs, there were twice as many dots in the transparent motion stimulus (4 dots/ $/ \mathrm{deg}^{2}$ of visual angle) as compared with the single surface used in Experiment 1. Third, single targets in a given stimulus dimension were always combined with neutral events in the other dimension. Fourth, six blocks 


\begin{tabular}{|c|c|c|c|c|c|c|c|}
\hline & \multicolumn{3}{|c|}{ Go signals } & \multicolumn{3}{|c|}{ No-go signals } \\
\hline & & Redundant & Single color & Single direction & Redundant & Single color & Single direction \\
\hline \multirow[t]{2}{*}{ Same surface } & Surface 1 & Red + right & Red + up & Right + blue & Green + left & Green + up & Left + blue \\
\hline & Surface 2 & No change & No change & No change & No change & No change & No change \\
\hline \multirow[t]{2}{*}{ Different surfaces } & Surface 1 & Red & Red & Right & Green & Green & Left \\
\hline & Surface 2 & Right & Up & Blue & Left & Up & Blue \\
\hline
\end{tabular}

Table 2. Experiment 2. One possible combination of go-, no-go, and neutral events for participants instructed to detect rightward motion (direction target) or the color red (color target). Single color signals were always accompanied by a neutral change in direction (upward motion); single direction signals were always presented together with a neutral change in color (blue). All events occurred either on the same surface or on different surfaces.

consisting of 120 trials each were run on two consecutive days. In a single block, there were 12 signal conditions: 6 go conditions comprising redundant targets with both signals in the same surface or in different surfaces, single color target + neutral direction event in the same surface or in different surfaces, and single direction target + neutral color event in the same surface or in different surfaces and also 6 no-go conditions comprising redundant no-gos in the same surface or in different surfaces, single color no-go + neutral direction event in the same surface or in different surfaces, and single direction no-go + neutral color event in the same surface or in different surfaces. See Table 2 for an overview of a possible combination of go, no-go, and neutral events in Experiment 2. Twelve participants (five men and seven women; ages $20-33, M=24.9, S D=3.4$ ) participated in Experiment 2. They were informed that the changes in color and direction of motion could either occur in the same surface or in different surfaces.

\section{Data analyses}

As before, redundancy gains were examined by comparing RTs to redundant targets with the fastest RTs to single targets. Mean RTs were computed for each combination of participant, target type (redundant target vs. single target), and target surface (same vs. different). The average of the faster of the two single-target conditions was calculated across participants, separately for each target surface condition. Statistical significance was evaluated with a twoway ANOVA involving the within-subjects factors target type (redundant target vs. "fastest single target") and target surface (same vs. different). Violations of the race-model inequality were evaluated as in Experiment 1.

\section{Results and discussion}

\section{Redundancy gain}

Across participants, performance reached $92 \%$ correct for the no-go trials and varied between $98 \%$ and $100 \%$ correct for the five go-conditions. Mean RTs across participants are shown in Figure 5. The ANOVA revealed a highly significant main effect of target type, $F(1,11)=$ 53.68, $M S E=192.67, p<.001$, indicating that responses to redundant targets were reliably faster than responses to the single targets (326 vs. $355 \mathrm{~ms}$ ). A $95 \%$ confidence interval for the main effect of target type indicates that redundancy gains are in the range of $20-38 \mathrm{~ms}$. Most importantly, neither the main effect of target surface $(p=.17)$ nor the interaction between target type and target surface $(p=.87)$ reached significance. This clearly shows that there is no difference in redundancy gains between changes occurring in the same surface $(29 \mathrm{~ms})$ and changes occurring in different surfaces $(30 \mathrm{~ms})$ in a transparent motion stimulus.

\section{Race-model inequality}

Mean CDFs across participants are shown in Figure 6. The upper panel represents conditions in which changes in color, direction of motion, or both occur in the same surface of a transparent motion stimulus. Contrasting the redundant-target CDF (pentagrams) with the "sum of single targets" CDF (circles) reveals a clear violation of the race-model inequality because the redundant-target CDF lies above and to the left of the "sum of single targets" CDF. Paired $t$ tests across participants at each of the 10 percentile

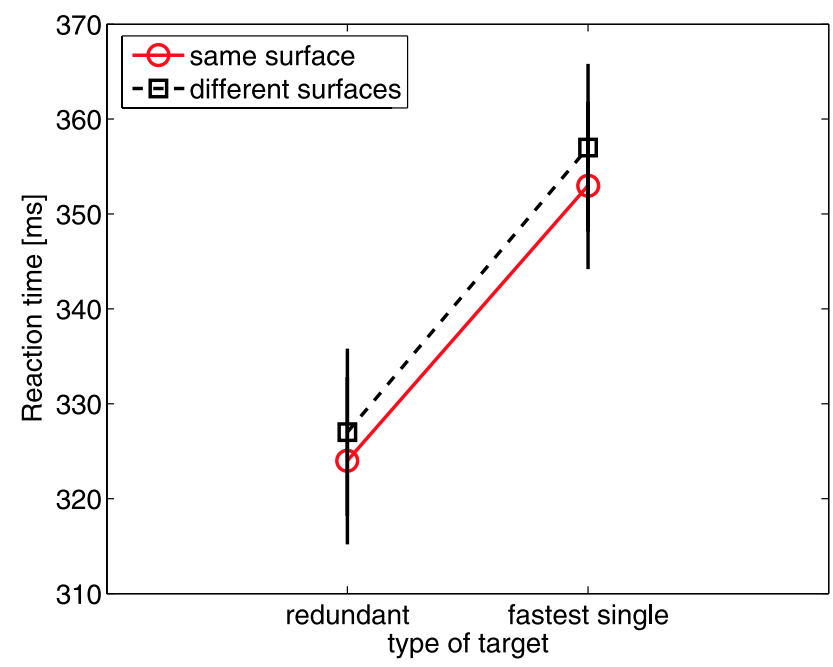

Figure 5. Experiment 2. RTs as a function of target type (redundant target vs. fastest single target) and target surface (same surface vs. different surfaces). RTs to redundant targets are faster than RTs to single targets. This effect does not depend on whether redundant targets are presented on the same surface or on different surfaces. Error bars indicate the $95 \%$ confidence interval for the main effect of target type (see Loftus \& Masson, 1994). 

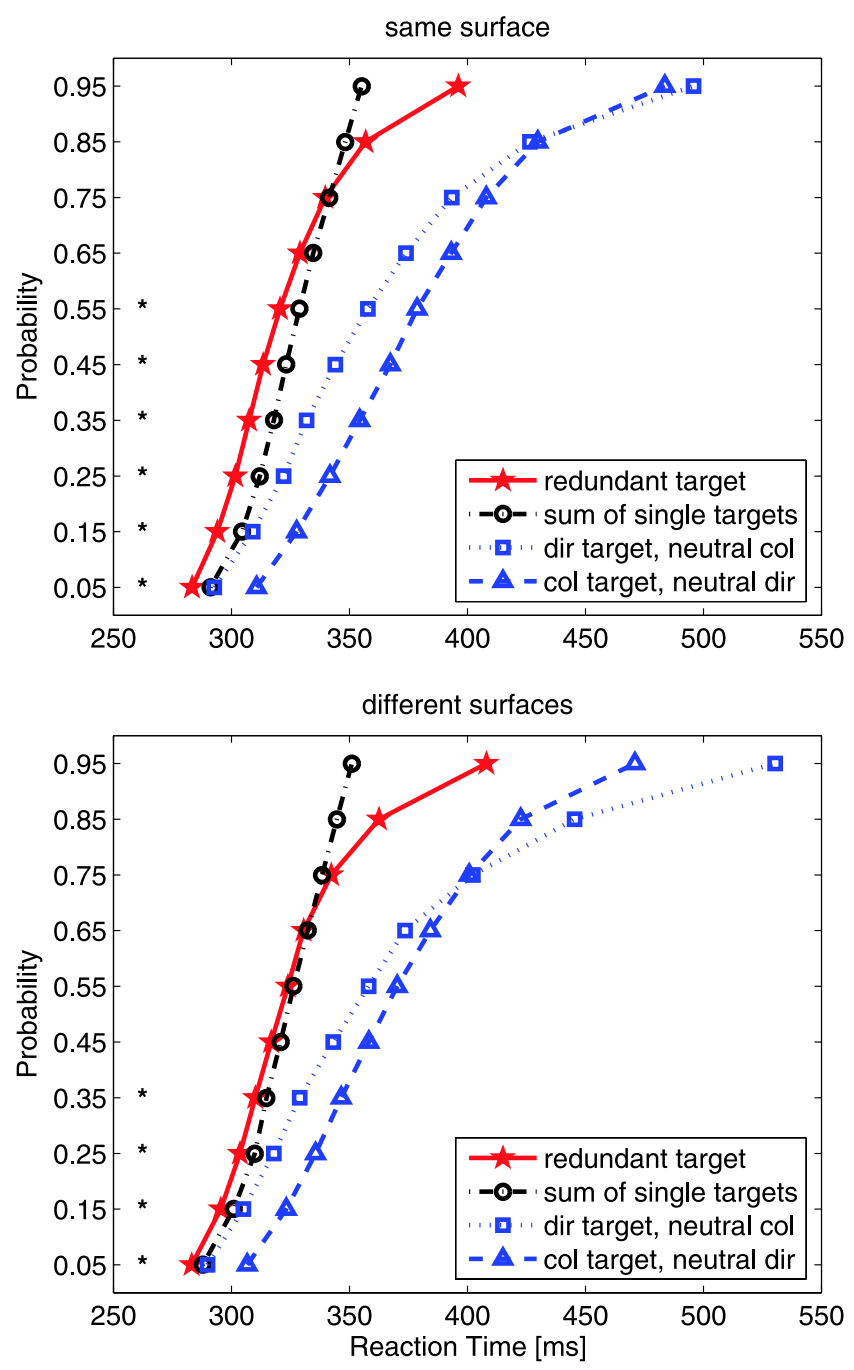

Figure 6. Experiment 2. Violations of the race-model inequality are revealed by comparing the redundant-target CDF (pentagrams) with the sum of the single-target CDFs (circles). Inconsistent with predictions of all race models, the redundant-target CDF lies above and to the left of the CDF for the sum of the single targets. This is the case not only if redundant targets occur on the same surface (upper panel) but also if they appear on different surfaces (lower panel). Significance markers along the $Y$-axis indicate the percentiles at which RTs to redundant targets were reliably faster than corresponding RTs for the sum of the single CDFs.

pairs confirmed that RTs from the redundant-target CDF were reliably faster than RTs from the "sum of single targets" CDF between the 5th and 55th percentiles $(.001<p<.002)$. Analogously, we compared the redundant-target CDF to the "sum of single targets" CDF in the condition where changes in color, direction of motion, or both occurred in different surfaces (lower panel in Figure 6). The race-model inequality was again considerably violated. RTs were reliably faster for the redundant-target $\mathrm{CDF}$ between percentiles 0.05 and $0.35(.01<p<.02)$.
The results of Experiment 2 show that color and motion signals are integrated, even if they occur in different surfaces of a transparent motion paradigm. This is evident from the fact that the magnitude of redundancy gains does not depend on whether two target signals occur in the same surface or in different surfaces. In addition, violations of the race-model inequality are prominent in both situations, suggesting integration of color and motion information across overlapping surface borders. Alternatively, one might argue that these two signals are integrated simply because they occur at the same location. Next, we tested whether integration of color and motion signals persists even if the two surfaces are positioned at different spatial locations.

\section{Experiment 3}

The purpose of Experiment 3 was to investigate whether the integration of color and motion signals across surfaces requires that the two surfaces are spatially overlapping. Instead of superimposing the two RDPs, we separated them spatially (Figure 1). If integration is restricted to a common spatial location, we should observe violations of the race-model inequality only if the target signals are presented at the same location but not if they are presented at different locations.

\section{Methods}

The basic go/no-go target detection paradigm was identical to Experiment 2 except for the following changes. The two RDPs were presented at spatially separate locations, centered 3.75 deg above and below fixation. Each of the 12 signal conditions was presented 12 times (6 go conditions: redundant targets with both signals in the same location or in different locations, single color target + neutral direction event in the same location or in different locations, and single direction target + neutral color event in the same location or in different locations; 6 no-go conditions: redundant no-go in the same location or in different locations, single color no-go + neutral direction event in the same location or in different locations, and single direction no-go + neutral color event in the same location or in different locations). Within each condition, three trials were used for each possible combination of the upper RDP's initial direction of rotation (clockwise vs. counterclockwise) and the location of the target event (upper vs. lower RDP). See Table 3 for a possible combination of go, no-go, and neutral events. Twelve participants (six men and six women; ages $20-27, M=23, S D=2.6$ ) were tested in Experiment 3. They were instructed to fixate on the central fixation square during the trials. Throughout each session, the experimenter monitored eye fixation with an infrared camera connected to a monitor outside the testing booth. 


\begin{tabular}{|c|c|c|c|c|c|c|c|}
\hline & \multicolumn{3}{|c|}{ Go signals } & \multicolumn{3}{|c|}{ No-go signals } \\
\hline & & Redundant & Single color & Single direction & Redundant & Single color & Single direction \\
\hline \multirow[t]{2}{*}{ Same location } & Location 1 & Red + right & Red + up & Right + blue & Green + left & Green + up & Left + blue \\
\hline & Location 2 & No change & No change & No change & No change & No change & No change \\
\hline \multirow[t]{2}{*}{ Different locations } & Location 1 & Red & Red & Right & Green & Green & Left \\
\hline & Location 2 & Right & Up & Blue & Left & Up & Blue \\
\hline
\end{tabular}

Table 3. Experiment 3. One possible combination of go-, no-go, and neutral events for participants instructed to detect rightward motion (direction target) or the color red (color target).

\section{Data analyses}

Redundancy gains were examined as before. Mean RTs were determined for each combination of participant, target type (redundant target vs. single target), and target location (same vs. different). For each participant, the faster of the two mean RTs in the single-target conditions was selected ("fastest single same" and "fastest single different," respectively). Statistical significance was evaluated with a two-way ANOVA involving the within-subjects factors target type (redundant target vs. fastest single target) and target location (same vs. different).

\section{Results and discussion}

\section{Redundancy gain}

Across participants, performance reached 97\% correct for the no-go trials and varied between $96 \%$ and $100 \%$ correct for the five go-conditions. Mean RTs across participants are shown in Figure 7. The ANOVA revealed a

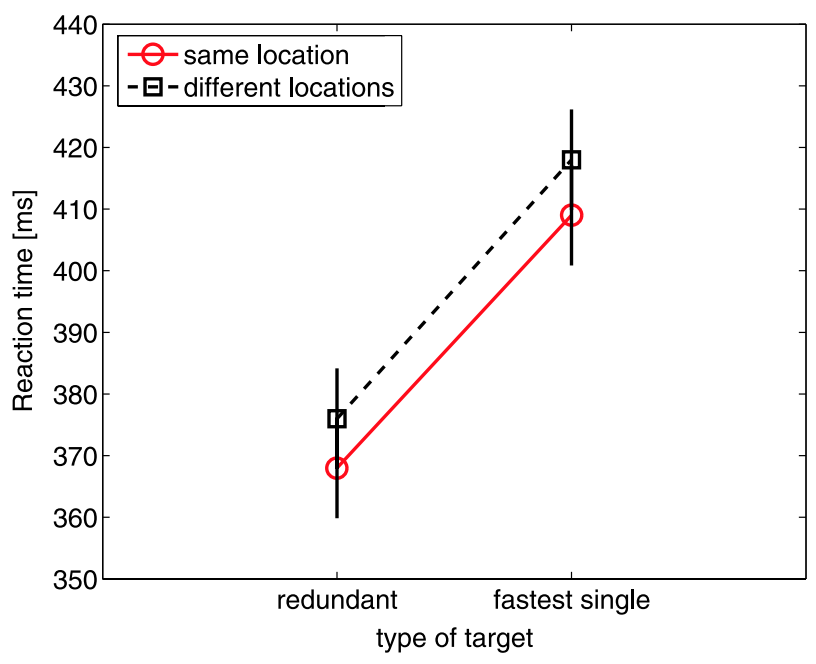

Figure 7. Experiment 3. RTs as a function of target type and target location. RTs to redundant targets are faster than RTs to single targets. RTs are also faster for events occurring at the same location than for those occurring at different locations. The absence of a significant interaction shows that the RTE does not depend on whether redundant targets are presented at the same location or at different locations. Error bars indicate the 95\% confidence interval for the main effect of target type (see Loftus \& Masson, 1994). highly significant main effect of target type, $F(1,11)=$ $125.74, M S E=165.08, p<.001$, indicating that responses to redundant targets $(372 \mathrm{~ms})$ were reliably faster than responses to the single targets $(414 \mathrm{~ms})$. A $95 \%$ confidence interval for the main effect of target type indicates that redundancy gains are in the range of 34-50 ms. The main effect of target location also reached significance, $F(1,11)=$ $7.15, M S E=118.51, p<.05$, indicating that responses to targets presented at the same location $(389 \mathrm{~ms})$ were faster than to targets presented at different locations (397 ms). Most important, however, was the absence of an interaction between target type and target location $(p=.8)$. This clearly shows that redundancy gains do not depend on whether the target events are presented at the same location (41 $\mathrm{ms})$ or at different locations (42 ms).

\section{Race-model inequality}

Mean CDFs across participants are shown in Figure 8. The upper panel represents conditions in which changes in color, direction of motion, or both occur at the same location. Paired $t$ tests across participants at each of the 10 percentile pairs confirmed that RTs from the redundanttarget CDF (pentagrams) were reliably faster than RTs from the "sum of single targets" CDF (circles) everywhere between the 5th and 55th percentiles $(.001<p<.05)$. The same comparison for the condition in which the target events occurred at different locations (lower panel in Figure 7) also revealed faster RTs for the redundant-target CDF everywhere between percentiles 0.05 and $0.65(.001<p<.05)$. Hence, in both cases, strong violations of the race-model inequality were observed.

These results show that redundancy gains and violations of the race-model inequality are prominent even if the two target events occur at separate spatial locations. It provides evidence for integration of color and motion signals across the visual field. To further assess the generality of this conclusion, we next tested whether the observed effects depend on the extent of spatial separation between the two stimuli.

\section{Experiment 4}

The purpose of Experiment 4 was to investigate whether the cross-dimensional integration observed in Experiment 3 is affected by the spatial distance between two RDPs. 

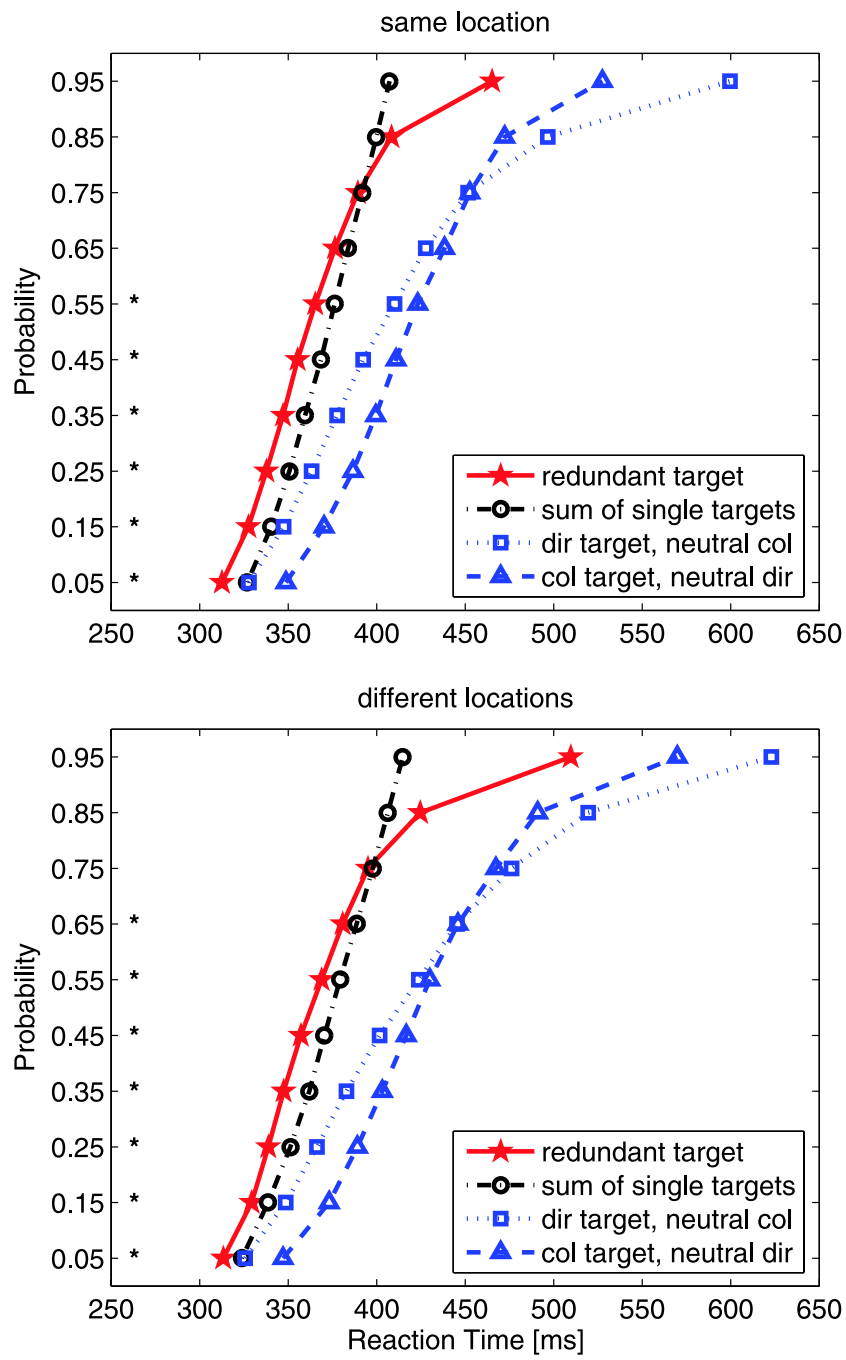

Figure 8. Experiment 3. Violations of the race-model inequality are revealed by comparing the redundant-target CDF (pentagrams) with the sum of the single-target CDFs (circles). Inconsistent with predictions of all race models, the redundant-target CDF lies above and to the left of the CDF for the sum of the single targets. This is evident irrespective of whether redundant targets occur at the same location (upper panel) or at different locations (lower panel). Significance markers along the $Y$-axis indicate the percentiles at which RTs were reliably faster for redundant targets than for the sum of single targets.

\section{Methods}

Methods and design were identical to Experiment 3 except for the spatial distance between the two RDPs, which were centered $7.5 \mathrm{deg}$ above and below fixation here (Figure 1c). To achieve this, we reduced the distance between participant and monitor to $57 \mathrm{~cm}$, resulting in a monitor resolution of 40 pixels/deg of visual angle. All stimulus properties were adjusted to ensure identity of the retinal image. Twelve participants (five men and seven women; ages $22-32, M=24.2, S D=2.9$ ) were tested in Experiment 4.
Redundancy gains and violations of the race-model inequality were evaluated as in the preceding experiments.

\section{Results and discussion}

\section{Redundancy gain}

Across participants, performance reached $97 \%$ correct for the no-go trials and varied between $92 \%$ and $100 \%$ correct for the five go-conditions. Mean RTs across participants are shown in Figure 9.

The ANOVA revealed a highly significant main effect of target type, $F(1,11)=159.18, M S E=100.50, p<.001$, indicating that responses to redundant targets were reliably faster than responses to the single targets (412 vs. $448 \mathrm{~ms}$ ). A 95\% confidence interval for the main effect of target type indicates that redundancy gains are in the range of 30-42 ms. The main effect of target presentation also reached significance, $F(1,11)=14.58, M S E=$ $178.27, p<.01$, indicating that responses to targets presented in the same location were faster than to targets presented in different locations (423 vs. $438 \mathrm{~ms}$ ). However, as in Experiment 3, the interaction between target type and target location did not reach significance $(p=.14)$, indicating that there is no difference in redundancy gains between changes in the same location ( $38 \mathrm{~ms}$ ) and changes in different locations $(31 \mathrm{~ms})$. It clearly shows that the magnitude of the redundancy gains is not affected by the spatial distance between the two stimuli. This conclusion is further supported by Miller (1982), who also reported

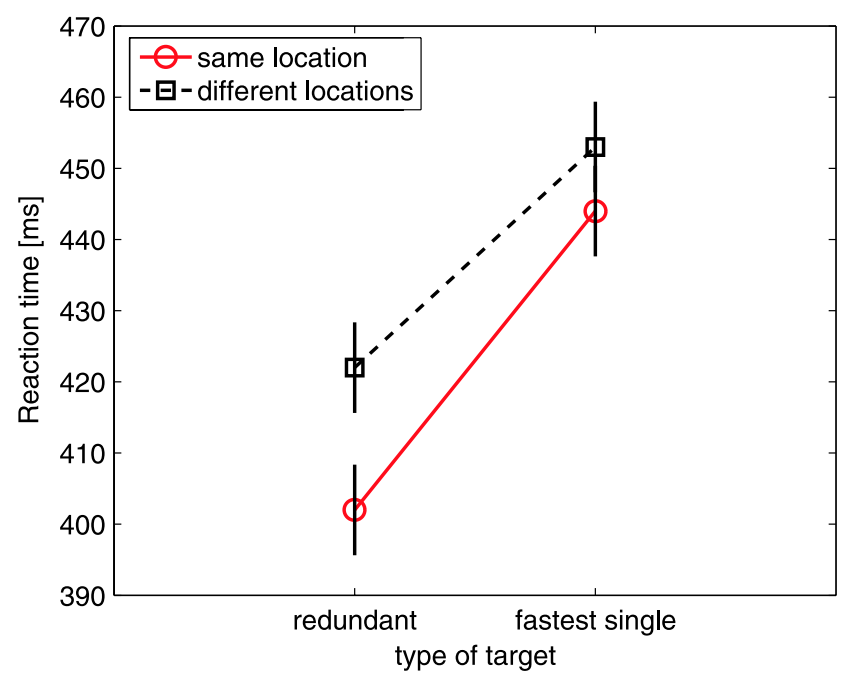

Figure 9. Experiment 4. RTs as a function of target type and target location. RTs to redundant targets are faster than RTs to single targets. RTs are also faster for events occurring in the same location than for those occurring in different locations. The absence of a significant interaction shows that the RTE does not depend on whether redundant targets are presented in the same location or in different locations. Error bars indicate the 95\% confidence interval for the main effect of target type (see Loftus \& Masson, 1994). 
the absence of distance effects in a different variant of the redundant-target paradigm.

\section{Race-model inequality}

Mean CDFs across participants are shown in Figure 10. Conditions in which changes in color, direction of motion, or both occur at the same location are shown in the upper panel. Paired $t$ tests across participants at each of the 10 percentile pairs confirmed that RTs from the redundant-
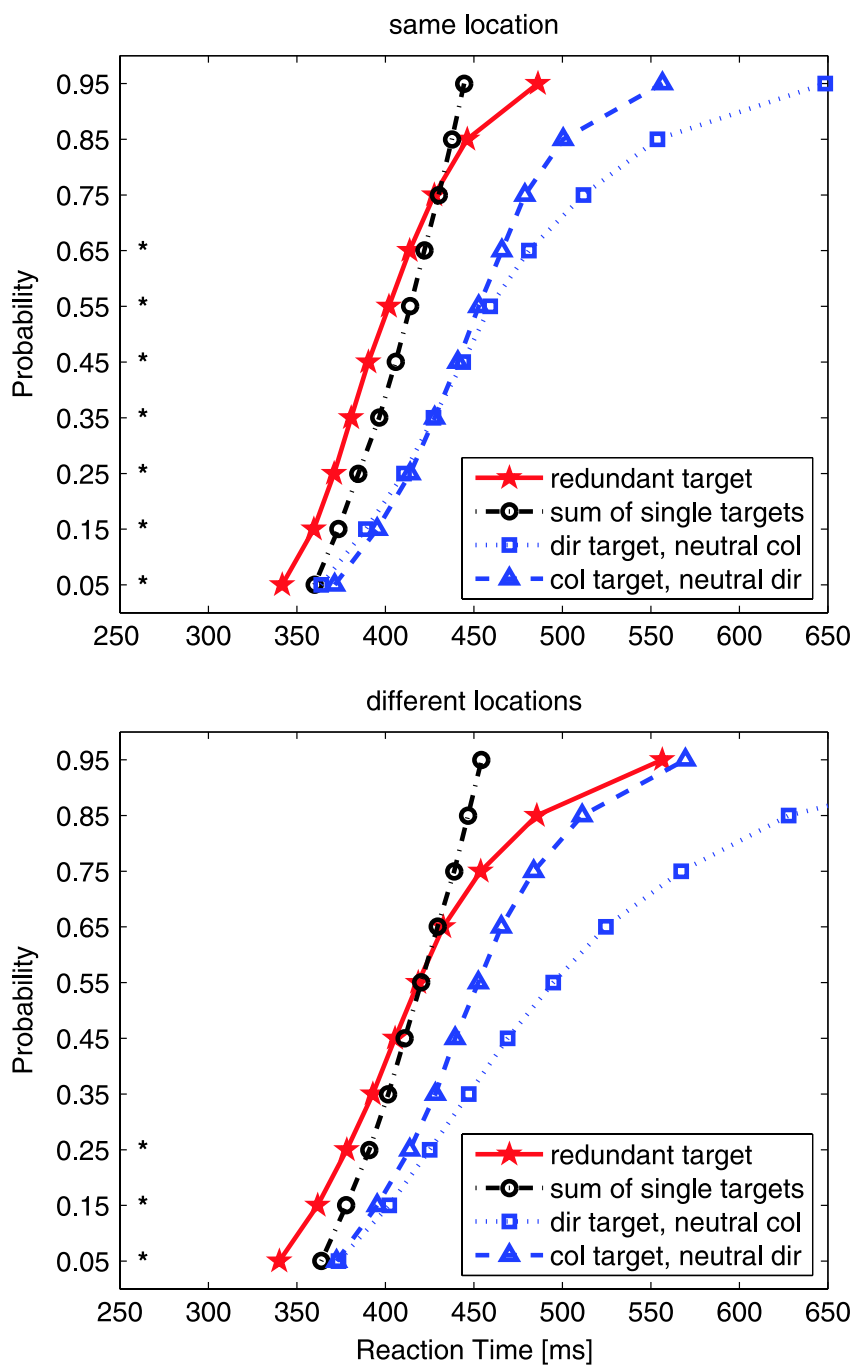

Figure 10. Experiment 4. Violations of the race-model inequality revealed by comparing the redundant-target CDF (pentagrams) with the sum of the single-target CDFs (circles). Inconsistent with predictions of all race models, the redundant-target CDF lies above and to the left of the CDF for the sum of the single targets. This is evident irrespective of whether redundant targets occur at the same location (upper panel) or at different locations (lower panel). It shows that the fastest response to redundant targets can be faster than the fastest responses to single targets. Significance markers along the $Y$-axis indicate the percentiles at which RTs were reliably faster for redundant targets than for the sum of single targets. target CDF (pentagrams) were reliably faster than RTs from the "sum of single targets" CDF (circles) everywhere between the 5th and 65th percentiles $(.001<p<.008)$. The lower panel of Figure 10 shows the condition in which the target events occurred at different locations. RTs for the redundant-target $\mathrm{CDF}$ were significantly faster between percentiles 0.05 and $0.25(.001<p<.01)$, with the difference at the 35 th percentile just closely failing to reach statistical significance $(p=.057)$. Hence, for targets at the same and at distant locations, strong violations of the race-model inequality were observed.

The outcome of Experiment 4 further supports the notion that color and motion information is integrated across stimuli presented at different locations in the visual field. Even when the stimuli are separated by 15 deg (center-tocenter distance), strong redundancy gains and reliable violations of the race-model inequality are observed.

\section{General discussion}

In four experiments, we investigated the cross-dimensional integration of color and visual motion signals by using the redundant-target paradigm. We presented moving RDPs in a speeded go/no-go target detection task, in which participants were required to respond to changes in the direction of motion (single target), color (single target), or both (redundant target). Experiment 1 established that redundancy gains are present for targets defined by their direction of visual motion and color: Responses to redundant targets were faster than responses to single targets. There was no difference between single targets presented alone and single targets that were combined with neutral events in the other stimulus dimension, which argues against the possibility that our redundancy gains are caused by inhibitory influences due to the presentation of a neutral event. Furthermore, the redundancy gains were inconsistent with predictions based on race models. We consider this as evidence that color and motion signals are integrated rather than processed separately and independently in this particular task. Experiment 2 shows that such integration is not restricted to a single surface in a transparent motion paradigm. Whereas redundant targets were presented either in the same surface or in different surfaces, integration of color and motion signals was revealed in both cases. Finally, Experiments 3 and 4 show that the integration of target information is independent of the spatial separation between the stimuli: Integration was observed irrespective of whether redundant targets were presented at the same location or at different locations, even if the spatial distance between the stimuli was as large as 15 deg. Taken together, this series of experiments demonstrates the integration of visual features that are represented in distinct visual areas across different stimulus constellations and task demands.

Our data allow us to draw conclusions about potential mechanisms of attention underlying performance in the 
redundant-target paradigm. Essentially, three different types of attentional mechanisms have been reported. Spatial attention refers to the ability to attend to a particular location in the visual field. All sensory information presented at the attended location is processed more efficiently than information outside the spatial focus of attention (Eriksen \& St. James, 1986; Posner, 1980). Feature-based attention, on the other hand, describes a mechanism by which attending to a particular feature (i.e., the color "red") enhances processing of only the attended feature, independent of the spatial focus of attention. Finally, object-based attention means that attention can be directed to perceptual groups or "objects" and that features of the same object are processed more efficiently than features belonging to different objects ("same-object advantage"; Blaser, Pylyshyn, \& Holcombe, 2000; Duncan, 1984; O’Craven, Downing, \& Kanwisher, 1999; for reviews, see Driver \& Baylis, 1998; Scholl, 2001). Strong evidence for object-based attentional mechanisms comes from studies in which two objects are superimposed, such that spatial location by itself cannot be used to orient attention. As for visual motion, superimposed objects are created by overlaying two RDPs moving coherently in opposite directions, thereby generating the percept of two surfaces sliding across each other. By assuming an objectbased mechanism of visual attention, one would predict that redundancy gains and violations of the race-model inequality should be obtained only for redundant targets occurring on the same but not on different surfaces (sameobject advantage). In contrast, we find strong redundancy gains and reliable violations of the race-model inequality in both conditions (Experiment 2), ruling out an object-based account of the effect. By assuming a spatial attentional mechanism, on the other hand, one would expect that redundancy gains and race-model violations should become evident for redundant targets occurring at the same location but not for different locations. However, the effects were consistently independent of spatial location (Experiments 3 and 4). Therefore, we propose that a feature-based attentional mechanism can best account for our findings. Several studies have demonstrated that observers can effectively attend to nonspatial stimulus features, and this has also been shown for color (Anllo-Vento \& Hillyard, 1996; Corbetta, Miezin, Dobmeyer, Shulman, \& Petersen, 1990; Sàenz, Buracas, \& Boynton, 2003) and direction of motion (Martínez-Trujillo \& Treue, 2004; Sàenz, Buracas, \& Boynton, 2002; Treue \& MartínezTrujillo, 1999; Treue \& Maunsell, 1996). In our task, participants have divided their attention between the target color and target direction, resulting in an enhanced processing of the designated target features throughout the visual field. The RTE has already been related to featurebased attention in the study by Mordkoff and Yantis (1993). In their variant of the redundant-target paradigm, they investigated integration of color and shape information and found violations of the race-model inequality not only if the two target elements were part of the same object (i.e., a colored letter) but also if they occurred at different spatial locations (i.e., a colored frame around a white letter or a colored patch below the letter). Yet, there are two important differences between these experiments and our approach. First, using moving RDPs allowed us to superimpose two objects and randomly present same-object and differentobject conditions without any change in stimulus attributes or perceptual task. Second, whereas Mordkoff and Yantis have demonstrated violations of the race-model inequality for the stimulus dimensions color and shape, we show such violations for the dimensions color and direction of motion.

At first sight, our results might seem to be difficult to reconcile with recent behavioral studies underscoring the importance of location, on the one hand, or objects, on the other hand, for performance in visual tasks. In particular, there are two different lines of research that both arrive at apparently discrepant conclusions: investigations of feature binding and work on object-based visual attention. Investigating feature binding, Holcombe and Cavanagh (2001), for example, have demonstrated that the stimulus dimensions color and orientation are correctly bound at very high rates of presentation but only if they were superimposed (i.e., presented at the same location). Why does location matter in the case of feature binding but not in our paradigm? We think that this discrepancy can be explained by differences in task demands. As Roskies (1999) has framed it, for feature binding, "one sort of visual feature [...] must be correctly associated with another feature [...] to provide a unified representation of that object." Such an explicit association is not a requirement in the type of divided-attention paradigm we have employed. In fact, because the two target features are not necessarily presented together in a given trial (single targets), an explicit association of the target features is impossible in those trials. Instead, detection of either target feature (direction of motion or color) is sufficient to initiate a response: As soon as, for example, the color red appears, a speeded response is required, regardless of the direction the red dots are moving. Likewise, the presence of, for example, rightward motion calls for an immediate response, irrespective of the color of the rightward moving dots. If we had intended to investigate the binding of color and motion, we would have asked the participants to indicate, for example, in which direction the red dots were moving. In such a situation, we would certainly expect location-based effects, that is, better performance if these two features were presented in the same location as compared with different locations. In our task, however, examining in which direction the red dots are moving might even have detrimental effects on RT. In the second line of research, transparent motion paradigms have been used to investigate object-based mechanisms of visual attention. Simultaneous judgments about speed and direction of motion are more accurate if they concern the same surface as opposed to different surfaces (Valdés-Sosa, Cobo, \& Pinilla, 1998). Furthermore, when a cue directs attention to one of two superimposed surfaces, subsequent changes in the direction of motion are discriminated less accurately in the uncued surface compared with the cued one (Mitchell, Stoner, Fallah, \& Reynolds, 2003; Reynolds, 
Alborzian, \& Stoner, 2003; Valdés-Sosa, Cobo, \& Pinilla, 2000). The underlying electrophysiological mechanism seems to be a selective reduction of visual neuronal responses to changes in the unattended surface, as evidenced by reduced early components of the event-related potential (ERP) (P1, N1; Valdés-Sosa, Bobes, Rodríguez, \& Pinilla, 1998). Given the considerable evidence for object-based attentional mechanisms with transparent motion stimuli, one might expect to find an indication of such mechanisms with the redundant-target paradigm as well. Just as in the case of feature binding, the object-based transparent motion studies we have mentioned place different demands on the visual system as compared with our task. Valdés-Sosa, Cobo, et al. (1998, 2000), Reynolds et al. (2003), and Mitchell et al. (2003) used designs that drew or directed attention to a given surface, resulting in prioritized processing of one surface over the other. Our design, in contrast, required participants to divide attention between the stimulus dimensions color and direction of motion, and not between two objects or surfaces. Because our targets were equally likely to appear in one surface or the other, participants would not benefit from allocating resources to one surface at the expense of the second one. Furthermore, as soon as the color turned, for example, red, a speeded response was required, regardless which surface was involved. To perform optimally in our task, participants would be well advised to just focus on, for example, the color red or some dots moving to the right and not to segregate the superimposed surfaces. Here as well, examining to which surface the target color belongs might be disadvantageous in terms of RT performance. Taken together, it seems justified to assume that differences in task demands can account for the apparent discrepancies between the various paradigms.

Alternatively, one could argue that these discrepancies might stem from the fact that we used speeded responses and compared RT distributions, whereas the other paradigms used threshold measurements (Holcombe \& Cavanagh, 2001) or percentage of correct responses (Reynolds et al., 2003; Valdés-Sosa et al., 2000) as their dependent variable. In particular, one might propose that feature binding (showing location-based effects) and attentional discrimination paradigms (showing object-based effects) directly probe mechanisms at early stages of visual processing, whereas RTs are influenced by many stages of processing between the retina and the motor cortex. However, it is not the case that RTs are insensitive to effects of perceptual integration and attention in early visual areas. For example, recent works on electrophysiological (Womelsdorf, Fries, Mitra, \& Desimone, 2005) and functional MRI (Weissman, Roberts, Visscher, \& Woldorff, 2005) have revealed a trial-by-trial correlation between stimulus-evoked activity in visual cortical areas and RT. Moreover, it has been shown that RTs also provide a signature for the presence or absence of feature binding, for example, in classic visual search studies (Treisman \& Gelade, 1980).

It is important for our approach, however, that the RTE is, at least partly, a perceptual effect and does not arise entirely at a premotor or motor level of processing. The locus of the RTE has been discussed controversially, with some studies advocating a premotor or motor contribution (e.g., Diederich \& Colonius, 1987; Giray \& Ulrich, 1993) but others arguing against it (Miller et al., 2001; Mordkoff et al., 1996). For instance, Giray and Ulrich (1993) measured response force in addition to RT. Response force, which is regulated in the motor cortex (Scott, 2003), was largest in redundant-target trials, leading the authors to propose contributions of motor areas to the RTE. However, Miller et al. (2001) provided direct evidence against a motor locus by analyzing single-cell recordings from primary motor cortex in nonhuman primates. Briefly, if the RTE originated during perceptual processing, the input signals to the motor cortex should already be speeded in redundant-target trials. This would be evident in shorter latencies (i.e., time differences between stimulus onset and onset of neuronal activity) of primary motor neurons in response to redundant targets compared with single targets. Alternatively, if the RTE arose at late motor levels of processing, the motor cortex output signals should show an additional redundancy gain. In this case, the difference between response latencies of primary motor neurons and corresponding RTs should be smaller for redundant trials compared with single signal trials. Miller et al. found a reduction in neuronal response latencies to redundant targets, although there was no difference between neuronal latencies and RTs directly disconfirming the hypothesis that late motor areas constitute the origin of the RTE. Moreover, evidence in favor of perceptual contributions to the RTE has been reported consistently by a number of studies. ERP recordings have demonstrated influences of redundant targets on components associated with early visual processing (N1, P1; Miniussi et al., 1998) or target selection (P2, N2p; Reimann, Müller, \& Krummenacher, 2004). Behaviorally, early sensory or perceptual contributions to the RTE have also been reported by Turatto et al. (2004) and Krummenacher et al. (2002), respectively. Taken together, these studies confirm that there is a substantial perceptual component to the RTE.

The functional integration of color and motion information has been investigated in a number of other paradigms, using behavioral, neurophysiological, and neuroimaging methods. Evidence for mostly independent processing of color and motion comes from psychophysical studies on temporal asynchronies in visual perception (Arnold \& Clifford, 2002; Arnold, Clifford, \& Wenderoth, 2001; Moutoussis \& Zeki, 1997; Nishida \& Johnston, 2002; Viviani \& Aymoz, 2001). Moutoussis and Zeki (1997) used moving objects that change their direction of motion and color. Although these changes would occur in perfect synchrony in some trials, they were shifted by different time lags with respect to each other in other trials. Psychophysical measures of the point of subjective synchrony revealed that motion changes have to happen $70-80 \mathrm{~ms}$ earlier than color changes for them to be perceived as occurring simultaneously. Exploiting the color-contingent motion aftereffect 
as an indirect measure of perceptual synchrony, Arnold et al. (2001) demonstrated a similar amount of processing lag for color and motion attributes of a stimulus. Such asynchronies in perception are taken as evidence for independent processing of color and motion signals and for a functional specialization of the visual brain areas (Zeki \& Bartels, 1998, but see also Bedell, Chung, Ogmen, \& Patel, 2003). In contrast, a number of studies have demonstrated functional interactions between the color and motion processing systems (for reviews, see Croner \& Albright, 1999a; Dobkins \& Albright, 1993a). If the two streams were functionally separate, the perception of a moving object should be impossible if object and background are isoluminant. This prediction has been rejected in behavioral experiments (Dobkins \& Albright, 1993b; Hawken, Gegenfurtner, \& Tang, 1994). Furthermore, corresponding neurophysiological studies have shown that neurons in the medial temporal area (MT), which is strongly implicated in the perception of visual motion (Salzman, Britten, \& Newsome, 1990; Salzman, Murasugi, Britten, \& Newsome, 1992), continue to signal the direction of motion of heterochromatic stimuli even under conditions of isoluminance (Dobkins \& Albright, 1994; Gegenfurtner et al., 1994; Saito, Tanaka, Isono, Yasuda, \& Mikami, 1989). Similarly, it has been demonstrated that color information can improve perceptual performance: Psychometric and neurometric detection thresholds in coherent motion displays are strongly reduced if dots carrying the motion signal and random noise can be segmented based on different but isoluminant colors (Croner \& Albright, 1997, 1999b). The chromatic influence on motion processing can be strong, particularly under conditions of low luminance contrast (Thiele, Dobkins, \& Albright, 1999, 2001), and is independent of attentional load (Thiele, Rezec, \& Dobkins, 2002). Whereas some studies have shown a more prominent contribution of color information to motion processing for stimuli modulated along the red-green cardinal axis in color space (i.e., with L- and M-cone input; Gegenfurtner et al., 1994; Ruppertsberg, Wuerger, \& Bertamini, 1993, but see also Lu, Lesmes, \& Sperling, 1999 for an alternative account), others have also documented reliable effects for stimuli modulated along the yellowish-violet axis (i.e., with S-cone input; Seidemann \& Newsome, 1999; Wandell et al., 1999). Taken together, these studies demonstrate that color information is available to the visual motion processing system. Likewise, it has been shown that some neurons in the ventral stream area V4, which is mainly specialized for the processing of orientation and color, are direction selective (Desimone \& Schein, 1987; Ferrera, Rudolph, \& Maunsell, 1994; Mountcastle, Motter, Steinmetz, \& Sestokas, 1987; Tolias, Keliris, Smirnakis, \& Logothetis, 2005). Together, these findings provide strong evidence for shared neuronal resources for color and motion processing across the two visual streams, potentially representing a neural substrate for the perceptual integration of color and visual motion signals.

Possibly, one could object that perceptual integration of color and motion signals is achieved entirely by dorsal stream processing in the present experiments. Although the colors used in our experiments were objectively isoluminant $\left(25 \mathrm{~cd} / \mathrm{m}^{2}\right)$, there might have been differences in perceived, subjective isoluminance, which might have been driving dorsal stream neurons. We argue that such an effect cannot explain the integration observed. In all experiments, four different colors were used (gray, red, green, and blue), of which only one was the designated target color. Thus, participants could not simply respond to changes in chromatic contrast or possible differences in subjective isoluminance. Considering the extremely low error rate, it seems highly unlikely that participants based their perceptual decisions on differences in subjective luminance, which, presumably, would have been much smaller than the differences in chromatic contrast. Moreover, if the dorsal stream simply integrated changes in direction of motion with changes in subjective isoluminance, no difference between redundant targets and direction targets combined with neutral color changes would be expected. In fact, our data demonstrate highly significant differences between these two conditions, ruling out such an interpretation of the results.

In conclusion, we demonstrate the integration of color and motion information in a speeded detection task using the redundant-target paradigm. All variants of race models proposing independent and separate processing of color and motion signals in such a task can be ruled out. This is in line with recent psychophysical and neurophysiological evidence for substantial interactions between the color and motion processing systems. We show that this integration persists throughout different stimulus constellations and task demands. With transparent motion stimuli, integration of color and motion signals occurs across overlapping object borders, ruling out object-based selection in such a design. Spatial separation of color and motion signals does not constrain their integration either, rejecting locationbased accounts of the effect. Feature-based theories of attentional selection, on the other hand, propose enhanced processing of the attended features throughout the visual field. Because color and motion signals neither have to be assigned to the same object nor to the same location to be integrated in the redundant-target paradigm, performance in this task can be best characterized by a feature-based mechanism of divided visual attention.

\section{Acknowledgments}

We thank Jeff Miller for valuable discussions and advice, Christoph Nothdurft for helpful comments on an earlier version of the manuscript, and Çaglar Akçay for testing the participants in Experiments 1 and 2.

Commercial relationships: none.

Corresponding author: Steffen Katzner.

Email: steffen.katzner@mail.gwdg.de.

Address: German Primate Center, Göttingen, Germany. 
References

Anllo-Vento, L., \& Hillyard, S. (1996). Selective attention to the color and direction of moving stimuli: Electrophysiological correlates of hierarchical feature selection. Perception \& Psychophysics, 58, 191-206. [PubMed]

Arnold, D. H., \& Clifford, C. W. (2002). Determinants of asynchronous processing in vision. Proceedings, Biological Sciences/The Royal Society, 269, 579-583. [PubMed]

Arnold, D. H., Clifford, C. W., \& Wenderoth, P. (2001). Asynchronous processing in vision: Color leads motion. Current Biology, 11, 596-600. [PubMed] [Article]

Bedell, H. E., Chung, S. T., Ogmen, H., \& Patel, S. S. (2003). Color and motion: Which is the tortoise and which is the hare? Vision Research, 43, 2403-2412. [PubMed]

Blaser, E., Pylyshyn, Z. W., \& Holcombe, A. O. (2000). Tracking an object through feature space. Nature, 408, 196-199. [PubMed]

Corbetta, M., Miezin, F. M., Dobmeyer, S., Shulman, G. L., \& Petersen, S. E. (1990). Attentional modulation of neural processing of shape, color, and velocity in humans. Science, 248, 1556-1559. [PubMed]

Croner, L. J., \& Albright, T. D. (1997). Image segmentation enhances discrimination of motion in visual noise. Vision Research, 37, 1415-1427. [PubMed]

Croner, L., \& Albright, T. D. (1999a). Seeing the big picture: Integration of image cues in the primate visual system. Neuron, 24, 777-789. [PubMed] [Article]

Croner, L., \& Albright, T. D. (1999b). Segmentation by color influences responses of motion-sensitive neurons in the cortical middle temporal visual area. The Journal of Neuroscience, 19, 3935-3951. [PubMed] [Article]

Desimone, R., \& Schein, S. J. (1987). Visual properties of neurons in area V4 of the macaque: Sensitivity to stimulus form. Journal of Neurophysiology, 57, 835-868. [PubMed]

Diederich, A., \& Colonius, H. (1987). Intersensory facilitation in the motor component? A reaction time analysis. Psychological Research, 49, 23-29.

Dobkins, K. R., \& Albright, T. D. (1993a). Color, luminance, and the detection of visual motion. Current Directions in Psychological Science, 2, 189-193.

Dobkins, K. R., \& Albright, T. D. (1993b). What happens if it changes color when it moves?: Psychophysical experiments on the nature of chromatic input to motion detectors. Vision Research, 33, 1019-1036. [PubMed]
Dobkins, K. R., \& Albright, T. D. (1994). What happens if it changes color when it moves?: The nature of chromatic input to macaque visual area MT. The Journal of Neuroscience, 14, 4854-4870. [PubMed] [Article]

Driver, J., \& Baylis, G. (1998). Attention and visual object segmentation. In R. Parasuraman (Ed.), The attentive brain (pp. 299-325). Cambridge, MA: MIT Press.

Duncan, J. (1984). Selective attention and the organization of visual information. Journal of Experimental Psychology: General, 113, 501-517. [PubMed]

Eriksen, C. W., \& St. James, J. D. (1986). Visual attention within and around the field of focal attention: A zoom lens model. Perception \& Psychophysics, 40, 225-240. [PubMed]

Felleman, D., \& Van Essen, D. C. (1991). Distributed hierarchical processing in the primate cerebral cortex. Cerebral Cortex, 1, 1-47. [PubMed]

Ferrera, V. P., Nealey, T. A., \& Maunsell, J. H. R. (1992). Mixed parvocellular and magnocellular geniculate signals in visual area V4. Nature, 358, 756-761. [PubMed]

Ferrera, V. P., Rudolph, K. K., \& Maunsell, J. H. (1994). Responses of neurons in the parietal and temporal visual pathways during a motion task. The Journal of Neuroscience, 14, 6171-6186. [PubMed] [Article]

Gegenfurtner, K. R., Kiper, D. C., Beusmans, J. M., Carandini, M., Zaidi, Q., \& Movshon, J. A. (1994). Chromatic properties of neurons in macaque MT. Visual Neuroscience, 11, 455-466. [PubMed]

Giray, M., \& Ulrich, R. (1993). Motor coactivation revealed by response force in divided and focused attention. Journal of Experimental Psychology: Human Perception and Performance, 19, 1278-1291. [PubMed]

Hawken, M. J., Gegenfurtner, K. R., \& Tang, C. (1994). Contrast dependence of colour and luminance motion mechanisms in human vision. Nature, 367, 268-270. [PubMed]

Holcombe, A. O., \& Cavanagh, P. (2001). Early binding of feature pairs for visual perception. Nature Neuroscience, 4, 127-128. [PubMed] [Article]

Iacoboni, M., \& Zaidel, E. (2003). Interhemispheric visuomotor integration in humans: The effect of redundant targets. The European Journal of Neuroscience, 17, 1981-1986. [PubMed]

Krummenacher, J., Müller, H. J., \& Heller, D. (2001). Visual search for dimensionally redundant pop-out targets: Evidence for parallel-coactive processing of dimensions. Perception \& Psychophysics, 63, 901-917. [PubMed]

Krummenacher, J., Müller, H. J., \& Heller, D. (2002). Visual search for dimensionally redundant pop-out targets: Redundancy gains in compound tasks. Visual Cognition, 90, 801-837. 
Loftus, G., \& Masson, M. (1994). Using confidence intervals in within-subjects designs. Psychonomic Bulletin \& Review, 1, 476-490.

Lu, Z. L., Lesmes, L. A., \& Sperling, G. (1999). The mechanism of isoluminant chromatic motion perception. Proceedings of the National Academy of Sciences of the United States of America, 96, 8289-8294. [PubMed] [Article]

Martínez-Trujillo, J. C., \& Treue, S. (2004). Feature-based attention increases the selectivity of population responses in primate visual cortex. Current Biology, 14, 744-751. [PubMed] [Article]

Miller, J. (1982). Divided attention: Evidence for coactivation with redundant signals. Cognitive Psychology, 14, 247-279. [PubMed]

Miller, J. (1986). Timecourse of coactivation in bimodal divided attention. Perception \& Psychophysics, 40, 331-343. [PubMed]

Miller, J. (2004). Exaggerated redundancy gain in the split brain: A hemispheric coactivation account. Cognitive Psychology, 49, 118-154. [PubMed]

Miller, J., Ulrich, R., \& Lamarre, Y. (2001). Locus of the redundant-signals effect in bimodal divided attention: A neurophysiological analysis. Perception \& Psychophysics, 63, 555-562. [PubMed]

Miniussi, C., Girelli, M., \& Marzi, C. A. (1998). Neural site of the redundant target effect electrophysiological evidence. Journal of Cognitive Neuroscience, 10, 216-230. [PubMed]

Mitchell, J., Stoner, G., Fallah, M., \& Reynolds, J. H. (2003). Attentional selection of superimposed surfaces cannot be explained by modulation of the gain of color channels. Vision Research, 43, 1323-1328. [PubMed]

Mordkoff, J. T., Miller, J., \& Roch, A. C. (1996). Absence of coactivation in the motor component: Evidence from psychophysiological measures of target detection. Journal of Experimental Psychology: Human Perception and Performance, 22, 25-41. [PubMed]

Mordkoff, J. T., \& Yantis, S. (1991). An interactive race model of divided attention. Journal of Experimental Psychology: Human Perception and Performance, 17, 520-538. [PubMed]

Mordkoff, J. T., \& Yantis, S. (1993). Dividing attention between color and shape: Evidence of coactivation. Perception \& Psychophysics, 53, 357-366. [PubMed]

Mountcastle, V. B., Motter, B. C., Steinmetz, M. A., \& Sestokas, A. K. (1987). Common and differential effects of attentive fixation on the excitability of parietal and prestriate (V4) cortical visual neurons in the macaque monkey. The Journal of Neuroscience, 7, 2239-2255. [PubMed] [Article]
Moutoussis, K., \& Zeki, S. (1997). A direct demonstration of perceptual asynchrony in vision. Proceedings: Biological Sciences / The Royal Society, 264, 393-399. [PubMed]

Nishida, S., \& Johnston, A. (2002). Marker correspondence, not processing latency, determines temporal binding of visual attributes. Current Biology, 12, 359-368. [PubMed] [Article]

O'Craven, K. M., Downing, P. E., \& Kanwisher, N. (1999). fMRI evidence for objects as the units of attentional selection. Nature, 401, 584-587. [PubMed]

Posner, M. (1980). Orienting of attention. The Quarterly Journal of Experimental Psychology, 32, 3-25. [PubMed]

Raab, D. H. (1962). Statistical facilitation of simple reaction times. Transactions of the New York Academy of Sciences, 24, 574-590. [PubMed]

Reimann, B., Müller, H. J., \& Krummenacher, J. (2004). Visual search for dimensionally redundant pop-out targets: Behavioral and electrophysiological evidence for parallel co-active processing and location specificity. 11th Annual Meeting of the Cognitive Neuroscience Society, San Francisco.

Reynolds, J. H., Alborzian, S., \& Stoner, G. (2003). Exogenously cued attention triggers competitive selection of surfaces. Vision Research, 43, 59-66. [PubMed]

Roskies, A. L. (1999). The binding problem. Neuron, 24, 7-9, 111-125. [PubMed] [Article]

Ruppertsberg, A. I., Wuerger, S. M., \& Bertamini, M. (1993). The chromatic input to global motion perception. Visual Neuroscience, 20, 421-428. [PubMed]

Sàenz, M., Buracas, G. T., \& Boynton, G. M. (2002). Global effects of feature-based attention in human visual cortex. Nature Neuroscience, 5, 631-632. [PubMed] [Article]

Sàenz, M., Buracas, G. T., \& Boynton, G. M. (2003). Global feature-based attention for motion and color. Vision Research, 43, 629-637. [PubMed]

Saito, H., Tanaka, K., Isono, H., Yasuda, M., \& Mikami, A. (1989). Directionally selective response of cells in the middle temporal area (MT) of the macaque monkey to the movement of equiluminous opponent color stimuli. Experimental Brain Research, 75, 1-14. [PubMed]

Salzman, C. D., Britten, K. H., \& Newsome, W. T. (1990). Cortical microstimulation influences perceptual judgements of motion direction. Nature, 346, 174-177. [PubMed]

Salzman, C. D., Murasugi, C. M., Britten, K. H., \& Newsome, W. T. (1992). Microstimulation in visual area MT: Effects on direction discrimination performance. The Journal of Neuroscience, 12, 2331-2355. [PubMed] [Article] 
Scholl, B. J. (2001). Objects and attention: The state of the art. Cognition, 80, 1-46. [PubMed]

Scott, S. H. (2003). The role of primary motor cortex in goal-directed movements: Insights from neurophysiological studies on non-human primates. Current Opinion in Neurobiology, 13, 671-677. [PubMed]

Seidemann, E., \& Newsome, W. T. (1999). Effect of spatial attention on the responses of area MT neurons. Journal of Neurophysiology, 81, 1783-1794. [PubMed] [Article]

Thiele, A., Dobkins, K. R., \& Albright, T. D. (1999). The contribution of color to motion processing in macaque middle temporal area. The Journal of Neuroscience, 19, 6571-6587. [PubMed] [Article]

Thiele, A., Dobkins, K. R., \& Albright, T. D. (2001). Neural correlates of chromatic motion perception. Neuron, 32, 351-358. [PubMed] [Article]

Thiele, A., Rezec, A., \& Dobkins, K. R. (2002). Chromatic input to motion processing in the absence of attention. Vision Research, 42, 1395-1401. [PubMed]

Tolias, A. S., Keliris, G. A., Smirnakis, S. M., \& Logothetis, N. K. (2005). Neurons in macaque area V4 acquire directional tuning after adaptation to motion stimuli. Nature Neuroscience, 8, 591-593. [PubMed]

Treisman, A. M., \& Gelade, G. (1980). A featureintegration theory of attention. Cognitive Psychology, 12, 97-136. [PubMed]

Treue, S., \& Martínez-Trujillo, J. C. (1999). Feature-based attention influences motion processing gain in macaque visual cortex. Nature, 399, 575-579. [PubMed]

Treue, S., \& Maunsell, J. H. (1996). Attentional modulation of visual motion processing in cortical areas MT and MST. Nature, 382, 539-541. [PubMed]

Turatto, M., Mazza, V., Savazzi, S., \& Marzi, C. A. (2004). The role of the magnocellular and parvocellular systems in the redundant target effect. Experimental Brain Research, 158, 141-150. [PubMed]
Ungerleider, L., \& Mishkin, M. (1982). Two cortical visual systems. In D. Ingle, M. Goodale, \& R. Mansfield (Eds.), Analysis of visual behavior (pp. 49-86). MA: MIT Press.

Valdés-Sosa, M., Bobes, M., Rodríguez, V., \& Pinilla, T. (1998). Switching attention without shifting the spotlight: Object-based attentional modulation of brain potentials. Journal of Cognitive Neuroscience, 10, 137-151. [PubMed]

Valdés-Sosa, M., Cobo, A., \& Pinilla, T. (1998). Transparent motion and object-based attention. Cognition, 66, B13-B23. [PubMed]

Valdés-Sosa, M., Cobo, A., \& Pinilla, T. (2000). Attention to object files defined by transparent motion. Journal of Experimental Psychology: Human Perception and Performance, 26, 488-505. [PubMed]

Viviani, P., \& Aymoz, C. (2001). Colour, form, and movement are not perceived simultaneously. Vision Research, 41, 2909-2918. [PubMed]

Wandell, B. A., Poirson, A. B., Newsome, W. T., Baseler, H. A., Boynton, G. M., Huk, A., et al. (1999). Color signals in human motion-selective cortex. Neuron, 24, 901-909. [PubMed] [Article]

Weissman, D. H., Roberts, K. C., Visscher, K. M., \& Woldorff, M. G. (2005). The neural correlates of momentary lapses of attention. Program No. 770.11. 2005 Abstract Viewer/Itinerary Planner. Washington, DC: Society for Neuroscience, 2005. Online.

Womelsdorf, T., Fries, P., Mitra, P. P., \& Desimone, R. (2006). Gamma-band synchronization in visual cortex predicts speed of change detection. Nature, 439, 733-736. [PubMed]

Zeki, S., \& Bartels, A. (1998). The autonomy of the visual systems and the modularity of conscious vision. Philosophical Transactions of the Royal Society of London. Series B, Biological Sciences, 353, 1911-1914. [PubMed] 


\subsection{Anticipation of impending signals lowers decision crite- rion without affecting perceptual sensitivity}

Effects of attention are not the only cognitive influence that modulate the processing of sensory information. Other well known cognitive factors include memory processes, experience and expectation. Regarding effects of signal anticipation, classical experiments have demonstrated that RTs are inversely related to the hazard rate, i.e. to the conditional probability that a response demanding signal will be presented in the next moment, given it has not appeared so far (Niemi and Näätänen, 1981). Recently, two neurophysiological experiments have shown that neuronal activity in areas V4 (Ghose and Maunsell, 2002) and LIP (Janssen and Shadlen, 2005) is modulated according to the hazard rate of signal appearance.

Based on the behavioral studies, two mechanisms could explain the observed effects: On the one hand, subjects could adjust their perceptual sensitivity according to the hazard rate, yielding faster RTs with increasing hazard rate because of improved perceptual processing. On the other hand, subjects could also lower their decision criterion, i.e. the readiness to respond, with increasing hazard rate, which would also lead to faster RTs. Since neuronal correlates of the hazard rate have been observed in both visual (V4, Ghose and Maunsell, 2002) and visuo-motor areas (LIP, Janssen and Shadlen, 2005) of the cortex, the neurophysiological studies cannot clarify this question either.

In this experiment, we employed a signal detection approach to disentangle adjustments of perceptual sensitivity from changes in the decision criterion. We find that signal anticipation mainly influences the decision criterion without affecting perceptual sensitivity. This result is further confirmed by a behavioral reverse correlation approach which shows that subjects rather change the amplitude than the overall shape of perceptual filters during changes of the hazard rate. 


\title{
Anticipation of Impending Signals Lowers Decision Criterion Without Affecting Perceptual Sensitivity
}

\author{
Steffen Katzner ${ }^{1,2}$, Laura Busse ${ }^{1,2}, \&$ Stefan Treue ${ }^{1,2,3}$ \\ ${ }^{1}$ Cognitive Neuroscience Laboratory, German Primate Center, Göttingen, Germany \\ 2 Bernstein Center for Computational Neuroscience, Göttingen, Germany \\ 3 Department of Biology, Georg-Elias-Müller Institute for Psychology, University of Göttingen, Germany
}

\begin{abstract}
An accurate prediction of upcoming events is crucial for the effective allocation of processing resources and for planning actions. Numerous behavioral studies have shown that reaction time is inversely related to the hazard rate, i.e. the conditional probability that a response-demanding signal is about to occur, given it has not occurred so far. Here, we used a combination of signal detection theory and a novel behavioral reverse correlation approach to investigate whether benefits of event predictability are mediated by improving perceptual sensitivity or by adjustments in decision-related variables over time. The data show that perceptual sensitivity $\left(d^{\prime}\right)$ is not affected by the hazard rate and does not change during a trial. In contrast, we observed a strong dependence of the decision criterion $(\beta)$ on the hazard rate. These results indicate that the human visual system actively tracks the probability of upcoming events by adjusting its internal decision criterion while maintaining a constant perceptual sensitivity.
\end{abstract}

\section{Introduction}

In the Seoul 1988 Olympics, the German decathlet Jürgen Hingsen, favorite for the gold medal, was disqualified for false-starting three times in the $100 \mathrm{~m}$ dash competition. Taking the enormous risk of being expelled from the Games can only be justified if performance is greatly enhanced by correctly anticipating the starter's gun. In fact, several laboratory experiments have demonstrated that human reaction time (RT) is faster if subjects can anticipate the point in time that a response demanding signal is likely to appear, compared to situations where such anticipation is impossible (Klemmer, 1956; Requin and Granjon, 1969). To correctly anticipate upcoming signals, an observers' brain must represent the conditional probability 
that a signal is about to appear, given it has not appeared yet. The resulting quantity is termed the hazard rate. Electrophysiological studies in non-human primates have recently revealed a positive correlation between the hazard rate and neuronal activity in two areas of visual cortex (medial temporal area (MT), Ghose and Maunsell (2002); lateral intraparietal area (LIP), Janssen and Shadlen (2005)). Given that these areas are specialized for sensory processing (MT), or represent an interface between sensory processing and motor planning (LIP), it seems possible that the hazard rate might influence sensitivity of visual processing. Here, we devised a behavioral paradigm to address the question whether the correct prediction of upcoming signals can lead to changes in sensory sensitivity or mainly causes adjustments in decision-related variables, hence coming secondary to sensory processing.

To investigate the benefits of correctly anticipating upcoming signals we used a seveninterval visual-motion signal detection paradigm, which allowed us to disentangle changes in perceptual sensitivity from changes in the decision criterion (Green and Swets, 1966). Subjects had to detect a single, threshold-level coherent motion signal (target) among consecutive, discrete presentations of brief random-motion dot stimuli (RDPs), separated by blank intervals (Fig. 1a). They were instructed to report the target as soon as they detected it. Predictability of an upcoming target was manipulated by using two different hazard conditions (Fig. 1b). In one block, the hazard rate was increasing from interval to interval such that the likelihood of future events increased with every interval passing by. In a second block, the hazard rate remained constant, and therefore the likelihood of instant target signal appearance did not vary with the number of past intervals. The discrete, seven-interval design we used offers the advantage to evaluate hits (i.e., correct positive responses) and false alarms (i.e., incorrect positive responses) for every single interval in the stimulus sequence. Adopting the framework of signal detection theory, we used these hits and false alarms to compute measures of perceptual sensitivity $\left(d^{\prime}\right)$ and decision criterion $(\beta)$ as a function of stimulus interval and hazard condition.

Moreover, assuming that a subject's response results from some perceptual filter being applied to the motion signal in the stimulus, we performed a noise-based estimation of this filter, or kernel, and examined how it changed from interval to interval, separately for each hazard condition. In this approach, the underlying idea is to characterize the linear relationship between certain stimulus properties and subsequent behavioral responses, from which mechanisms of sensory processing, i.e., perceptual filters, can then be inferred. In this context, changes in the overall shape of the perceptual filter would indicate changes in perceptual sensitivity. In contrast, multiplicative scaling of the filter, without significant changes in its overall shape, would be consistent with changes in the decision criterion (Eckstein et al., 2002). For this approach, we adapted the reverse correlation technique that has been used in neurophysiological studies (Cook and Maunsell, 2004).

The signal detection analysis showed that perceptual sensitivity remains unchanged from interval to interval, independent of the hazard condition. In contrast, the decision criterion is strongly lowered from interval to interval, but only if the hazard rate is increasing. This clearly indicates that the behavioral benefit of correctly anticipating relevant signals is mediated by 
A

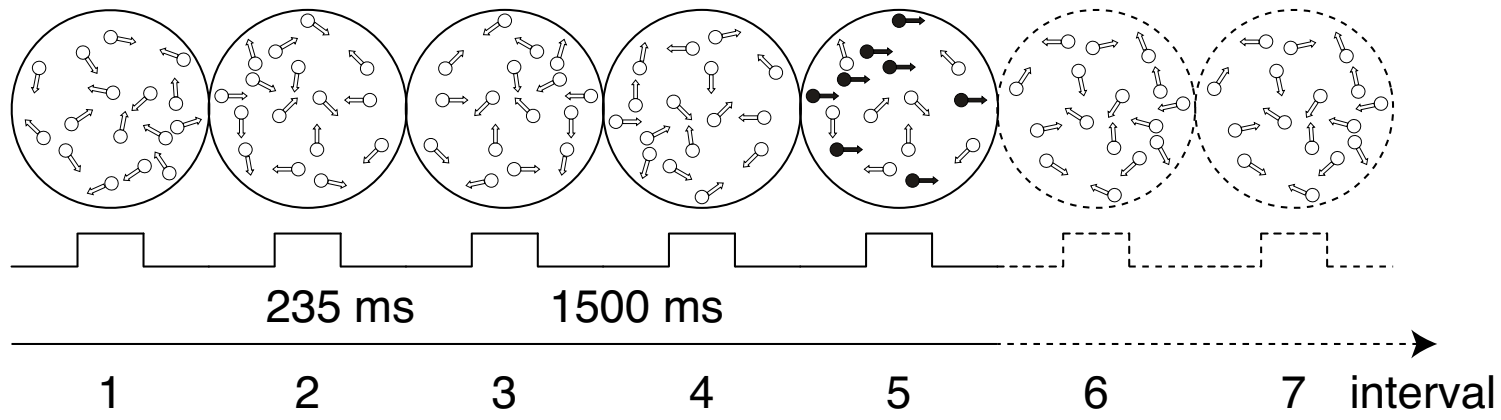

B Uniform

Geometric
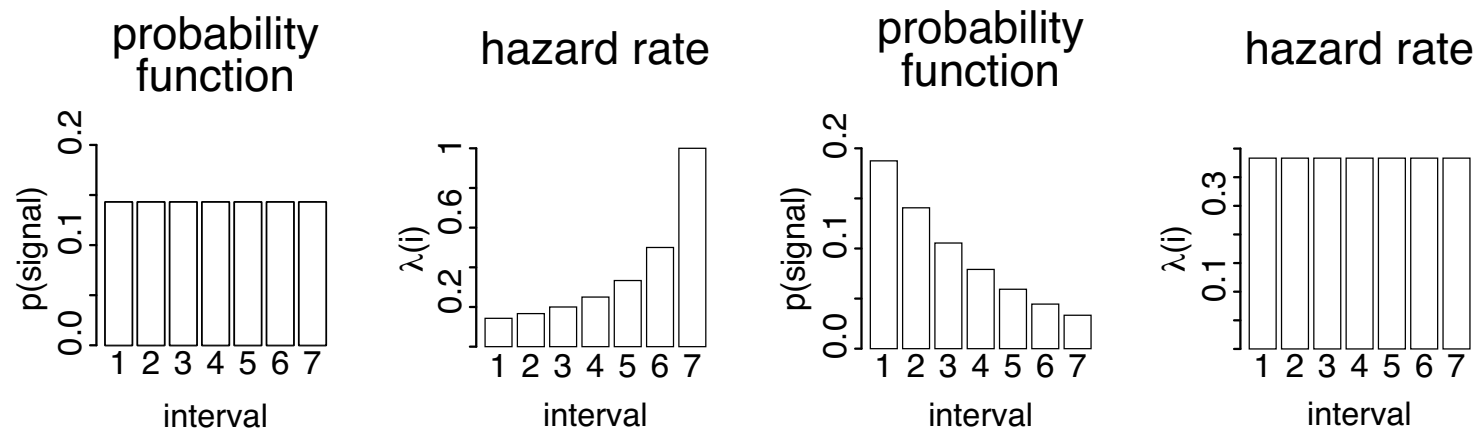

Figure 1: Methods. (a) Sequence of events in a single trial. Subjects viewed a temporal sequence of 1-7 brief presentations of a centrally displayed, moving random dot pattern, followed by a blank screen. The subject's task was to press a button upon detection of a threshold-level coherent motion signal of a pre-defined direction, embedded in a sequence of otherwise random noise stimuli ( $0 \%$ coherence). Trials were terminated after the response (hit or false alarm), but also if the target had been presented and no response was given during the following interstimulus-interval (miss). Subjects received auditory feedback after each trial. For illustrative purposes, the target signal in the figure consists of rightward motion, and was presented in the fifth interval (signal and noise dots were indistinguishable in the actual experiment). Dotted lines indicate parts of the trial sequence that were not actually shown since the trial had already been terminated. (b) Target-interval distributions and corresponding hazard rates in the two different hazard conditions. In different blocks of trials, the stimulus interval containing the threshold-level coherent motion signal was drawn from a uniform or geometric distribution, respectively. While the uniform probability distribution yields an increasing hazard function, the hazard function of the geometric probability distribution remains constant across intervals. Each subject performed 9 runs containing 53 trials under each of the two hazard condition, with the order of conditions being counterbalanced across subjects. The subjects were neither informed about the different signal interval distributions nor about the change in the hazard conditions. 
changes in the decision criterion and not by changes in perceptual sensitivity. Consistent with this finding, our behavioral reverse correlation revealed that the perceptual filters did not qualitatively change their shape from interval to interval. Furthermore, an interval-dependent multiplicative scaling of the filter tuning seemed evident, and this scaling was more reliable for the increasing than for the constant hazard rate. Taken together, these results indicate that the human visual system dynamically updates the probability of upcoming events by adjusting its internal decision criterion while maintaining a constant perceptual sensitivity.

\section{Results}

\section{Signal Detection Analysis}

We obtained measures of perceptual sensitivity and response criterion for each signal interval and hazard condition using Signal Detection Theory (Green and Swets, 1966) (see Methods). The results are summarized in Fig. 2. In both hazard conditions, perceptual sensitivity $\left(d^{\prime}\right)$ remained unchanged across intervals of stimulus presentation (ANOVA, $P=0.18$ ), and there was no difference in the overall pattern of $d^{\prime}$ values between the two hazard conditions (ANOVA, $P=0.38$ ). In contrast, the response criterion $(\beta)$ strongly varied between stimulus intervals, and this variation depended on which hazard condition was used (ANOVA, $P<$ 0.002). While $\beta$ remained unaffected for the constant hazard rate (ANOVA, $P=0.8$ ), it strongly decreased across intervals for the increasing hazard rate (ANOVA, $P=0.003$, linear trend analysis, $P<0.0001)$. For the first intervals, changes in $\beta$ were more pronounced before leveling off towards the end of the sequence (quadratic trend analysis, $P=0.04$ ).

\section{Reverse correlation analysis}

Next, we performed a noise-based reverse correlation analysis to estimate the linear filter which, applied to the motion stimulus, best characterizes the subjects' responses for each hazard condition and interval. For this analysis, we only used stimuli containing purely random motion, i.e., stimuli that subjects incorrectly responded to ('false alarms'), or stimuli that subjects correctly did not respond to ("correct rejections"). Due to the random placement and motion direction of dots, the effective motion signal in such stimuli varies from trial to trial. To capture the effective motion signal in each noise stimulus we first computed, from frame to frame, every possible motion vector along which every single dot could have moved (Barlow and Tripathy, 1997; Cook and Maunsell, 2004). Second, we constructed a histogram of the obtained motion vectors, ranging from 0 to $360^{\circ}$, with a bin size of $30^{\circ}$. In order to reveal the relationship between stimuli of varying effective motion signals and subsequent behavioral responses, we performed a logistic multiple regression analysis to predict, on the basis of the binned effective motion signals in the random noise stimuli, false alarms and correct rejections, 

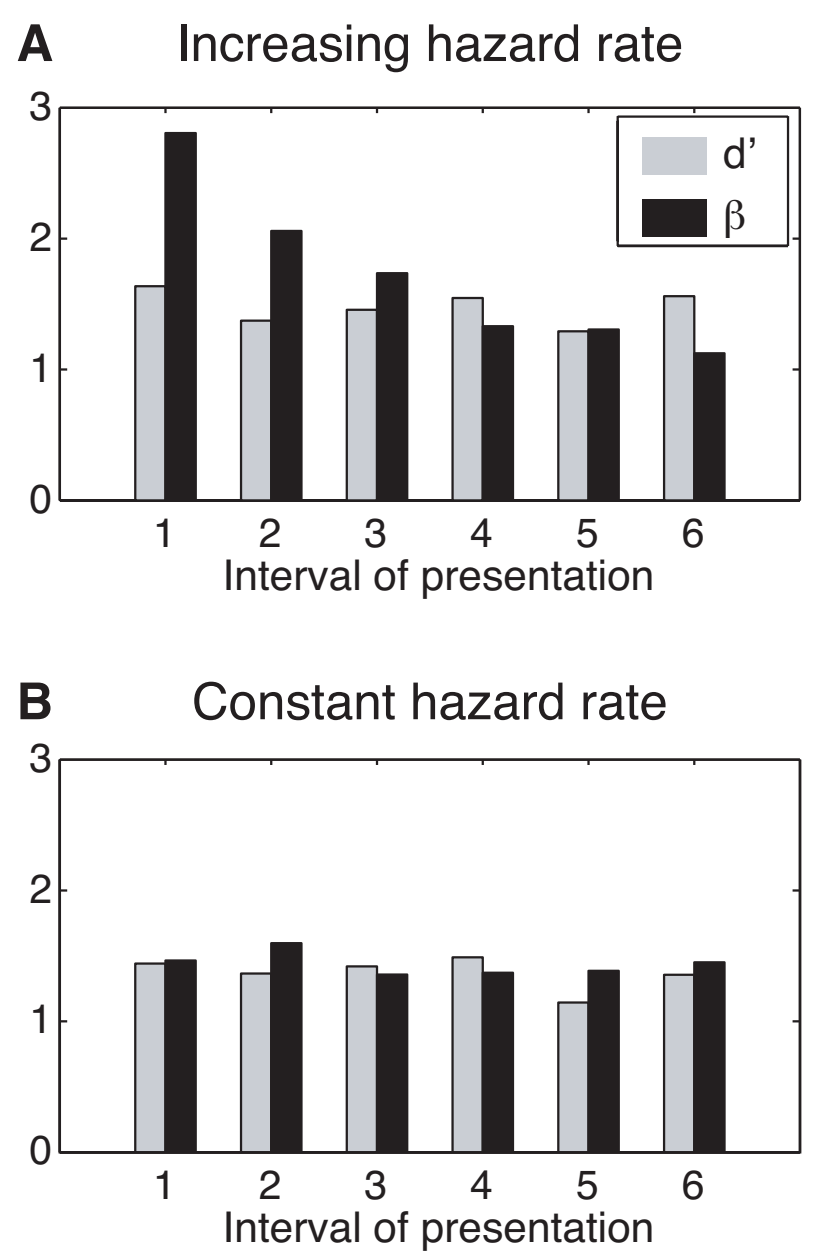

Figure 2: Results from signal detection analysis. Separately for the increasing (a) and constant (b) hazard condition, we calculated perceptual sensitivity $\left(d^{\prime}\right)$ and response criterion $(\beta)$ for each stimulus interval. Perceptual sensitivity is not influenced by the hazard condition, nor does it vary across intervals. In contrast, the response criterion strongly decreases across intervals, but only in the condition with increasing hazard rate. The decrease is most pronounced in the first four intervals. These results clearly show that benefits of signal anticipation are mediated by changes in the response criterion rather than by changes in perceptual sensitivity. 


\begin{tabular}{lllll}
\hline \hline & \multicolumn{4}{c}{ Hazard rate } \\
\cline { 2 - 5 } & \multicolumn{2}{c}{ constant } & \multicolumn{2}{c}{ increasing } \\
\cline { 2 - 5 } & \multicolumn{1}{c}{$\mathrm{n}$} & \multicolumn{1}{c}{$\mathrm{n}$} & $\mathrm{p}$ \\
\cline { 2 - 5 } Interval 1 & 2112 & $1.11 * 10^{-16}$ & 2417 & $6.16 * 10^{-6}$ \\
Interval 2 & 1262 & $1.08 * 10^{-8}$ & 1919 & $4.44 * 10^{-16}$ \\
Interval 3 & 778 & $4.68 * 10^{-10}$ & 1353 & $5.87 * 10^{-8}$ \\
Interval 4 & 458 & $3.24 * 10^{-14}$ & 842 & $5.57 * 10^{-11}$ \\
\hline \hline
\end{tabular}

Table 1: Summary of logistic regression models. A penalized maximum-likelihood algorithm (Firth, 1993) was used to fit a multiple logistic regression model to the subjects' behavioral responses (correct rejections or false alarms) triggered by random variations in the motion components of the preceding noise stimulus. While the number of observations $(n)$ necessarily decrease with increasing intervals, the model provides a very good description for the first four intervals ( $p$-values indicate the significance of the regression coefficients as determined by a Likelihood ratio test).

respectively. The resulting regression coefficients can be expressed as linear filter weights, and we estimated these weights separately for each interval in each hazard condition. In multiple regression, reliable estimates of the regression coefficients generally require a sufficient number of observations. Since the number of false alarms and correct rejections necessarily decreases towards the end of our stimulus sequence, we restricted the regression analysis to the first four intervals, combining the data from all subjects. Note also that decreases in $\beta$ are most pronounced for the first four intervals only (see Fig. 2). Table 1 summarizes the number of observations together with the validity of the regression models for the first four intervals in both hazard conditions.

Fig. 3a shows the kernel weights as estimated by the logistic regression analysis, after they have been smoothed with a Gaussian filter (width of 1 bin). If benefits of target signal anticipation are mediated by adjustments in sensory processing, we should observe pronounced changes in the kernels' overall shape from interval to interval. For instance, improved sensory performance could be achieved by increasing the weights for the target direction while decreasing the weights for other directions. In contrast, if benefits of target signal anticipation are mainly due to non-sensory, decision-related adjustments, we should observe multiplicative scaling only, without major changes in the kernel's shape, i.e., an increase of the weights for all directions by a constant factor. As can be seen in Fig. 3a, the general shape of the kernels does not change much from interval to interval, and this holds true for both hazard conditions. To evaluate changes in shape statistically, we scaled the kernel in each interval by a constant factor, giving the best fit to the kernel in the subsequent interval. With a true multiplicative scaling, the kernel for a given interval should be perfectly predictable by scaling the preceding kernel with the corresponding factor. However, because of noise in the estimation process this will never be the case, but the variance in the residuals can be used to assess the goodness of 


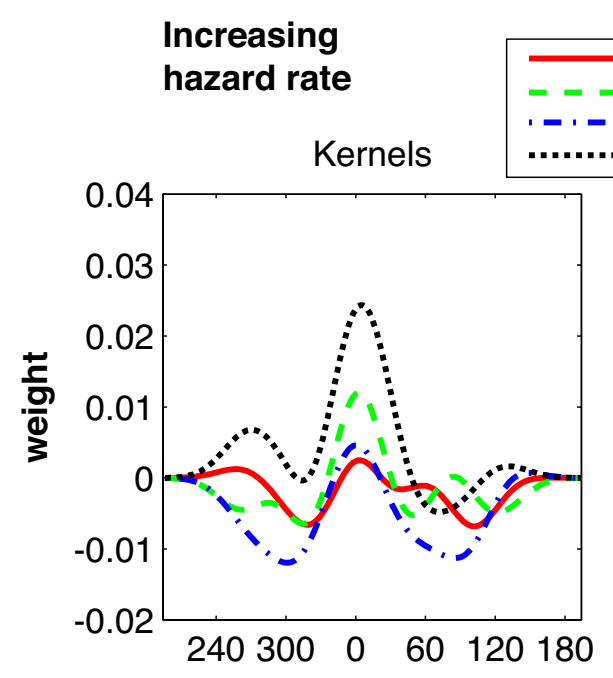

\section{Constant hazard rate}

Kernels
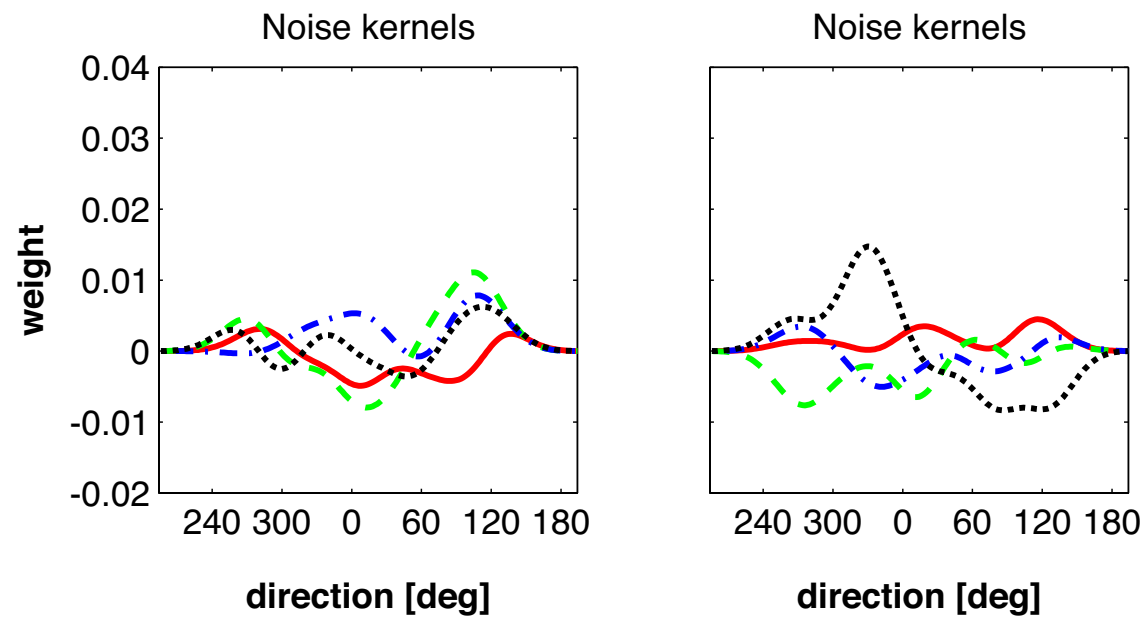

Figure 3: Results from behavioral reverse correlation analysis. The top panels show the kernels estimated by combining data from 7 subjects for the increasing (left) and constant (right) hazard condition, the bottom panel the corresponding noise kernels computed forward in time. In each condition, the signal direction $\left(0^{\circ}\right)$ closely corresponds to the point with highest amplitude. Differences between kernels in successive intervals can be attributed to multiplicative scaling without significant changes in kernel shape (but see discussion). Kernel amplitudes, i.e. the differences between maximal and minimal kernel weight, generally increase from interval to interval in the increasing hazard condition, which is in accord with decreases in the response criterion. Remarkably, the kernel for the first interval is almost flat in the increasing hazard condition, while it is much more pronounced in the constant hazard condition. This is in close agreement with the strong difference in response criteria for this interval between the two hazard conditions. 
the match between the actual and the predicted kernel. If the residual variance is much more pronounced relative to the variance expected by chance, this would reject the hypothesis of multiplicative scaling. To estimate residual variance obtained by chance, we computed 'noise kernels' by correlating false alarms and correct rejections with stimuli that have never been shown, or have only been shown in future trials, relative to the current behavioral response. Such noise kernels can be seen in Fig. 3b. Theoretically, these noise kernels should be flat since motion signals in the stimuli entering the prediction equations cannot be related to the current behavioral response. Therefore, profiles of noise kernels deviating from a flat line reflect the noise inherent in our kernel estimation process. Apparently, most of the noise kernels have a non-flat shape and it is therefore not surprising, that none of the statistical tests for deviations from multiplicative scaling reached significance ( $p>0.90$ for all comparisons). Hence, this finding has to be interpreted in light of the enormous variance that is present in the noise kernels (see discussion).

We also examined whether any interval-dependent scaling of the kernels was more pronounced for the constant than for the increasing hazard rate. As a measure of the strength of tuning for these kernels we computed the peak-to-peak amplitude, separately for each interval and hazard condition. Using a bootstrap-based analysis (Efron and Tibshirani, 1993), we found a significant positive increase in peak-to-peak amplitude as a function of interval for the increasing hazard rate $(P=0.03)$. While the peak-to-peak amplitudes for the constant hazard rate also tend to increase, this effect closely failed to reach statistical significance $(P=0.078)$. To validate that the increase in amplitudes is not caused mainly by an increase in noise, we calculated the signal-to-noise ratio, defined as the variance of the kernel divided by the variance of the corresponding noise kernel for each interval. Interestingly, for the increasing hazard rate, the signal-to-noise ratio increases across intervals, while it decreases only in the constant hazard rate condition. Taken together, our noise-based reverse-correlation analysis revealed kernels, or perceptual tuning curves, that expressed a distinct peak and a Mexican-hat shaped profile. Evidently, the profiles of these kernels seem to reflect changes in the hazard rate. While the kernel in the first interval is almost flat for the increasing hazard rate, the corresponding kernel for the constant hazard rate is much more pronounced in the corresponding first interval. Moreover, the scaling of the amplitudes seems to be more obvious for the increasing than for the constant hazard rate. However, because of the noise inherent in our kernel estimation procedure we are unable to draw firm conclusions as to whether the scaling is truly multiplicative or not.

\section{Discussion}

In this experiment, we addressed the question whether benefits of signal anticipation are mediated by changes in perceptual sensitivity or by adjustments of decision-related variables.

We manipulated the predictability of a pre-defined threshold-level target signal in a sequence of noise stimuli with increasing and constant hazard rates, respectively. With an increasing 
hazard rate, the immediate appearance of the target signal becomes more and more likely, given it has not appeared yet. In contrast, with constant hazard rates the conditional probability of instantaneous target signal appearance remains constant. Using signal detection theory we find that the magnitude of predictability of an upcoming target signal strongly influences the response criterion $(\beta)$, while it leaves perceptual sensitivity $\left(d^{\prime}\right)$ unaffected. This shows that benefits of successful signal anticipation are mediated by adjustments in decisionrelated variables, rather than changes in sensory processing. From reaction time research it has long been known that RT strongly depends on the variability and distribution of the 'fore-period', i.e., the time elapsing between the presentation of a warning stimulus and a response demanding stimulus (Klemmer, 1956; Drazin, 1961; Bertelson and Tisseyre, 1968; Requin and Granjon, 1969; Nickerson and Burnham, 1969; Niemi and Näätänen, 1981). RTs considerably decrease with longer fore-periods, but only if the immediate presentation of the response demanding signal becomes more and more likely over time. In contrast, RTs remain constant in the absence of such predictability. While this shows an influence of the magnitude of predictability of upcoming signals on behavior, such a measure cannot distinguish between sensory versus decision-related adjustments in stimulus processing. Unlike previous work, our discrete-interval signal detection approach offers the advantage to disentangle these alternatives, shedding light on the mechanism involved in target signal anticipation.

Various electrophysiological studies in non-human primates have investigated the neuronal basis of decision making. In particular, neurons in the lateral intra-parietal (LIP) cortex have been shown to play a major role in decision processes that guide behavior (Hanks et al., 2006; Platt and Glimcher, 1999), and their activity is strongly modulated by the hazard rate (Janssen and Shadlen, 2005). Hence, hazard-rate dependent modulations in the activity of area LIP might represent the neuronal basis for the variations in response criterion we observed.

In addition to the signal detection analysis, we adapted the reverse correlation technique for an analysis of behavioral data to assess how estimates of perceptual filters are influenced by different hazard rates. We reverse correlated behavioral responses with preceding stimuli to estimate kernels describing the subjects' weighting of the motion signal as a function of hazard rate and time. Reverse correlation has first been used in electrophysiological studies to investigate receptive field structures of sensory neurons (Sakai et al., 1988; Ringach and Shapley, 2004), and has subsequently been adopted for characterizing human observer templates in psychophysical studies (for a review, see Neri and Levi, 2006). While appropriate methods, e.g. classification image techniques, are available for psychophysical reverse correlation approaches with stationary white-noise stimuli, application of these techniques to visual motion is not straightforward because of autocorrelations in the stimulus. Other work has evaded this problem by using an equivalent linear regression approach (Ahumada Jr. and Lovell, 1971; Cook and Maunsell, 2004; DiCarlo et al., 1998; Theunissen et al., 2001; Blake and Merzenich, 2002). However, in none of these studies the dependent variable consisted of binary outcomes. Here, we used a logistic multiple regression approach which allowed us to reverse correlate binary behavioral outcomes, (i.e., false alarms or correct rejections) with the motion signal in the stimulus preceding that response. The validity of our approach is strongly supported 
by the significance of the regression model fits (see Table 1). Since the logistic regression approach can be applied in virtually any detection task context, this method is a useful addition to previous implementations of psychophysical reverse correlation.

Reverse correlation critically depends on random variations in a noisy stimulus that are correlated with observed responses. We introduced noise into moving random dot patterns by randomly assigning different directions of motion and speeds to individual dots. While speed was always kept constant for all dots across all trials, it would be optimal to vary the motion direction of individual dots randomly for every single trial. However, our stimulus-generating software currently does not allow this. While the starting positions of individual dots vary across trials, the random direction assigned to each dot is constant throughout a block of trials. Consequently, the distribution of possible motion vectors varies much more strongly across blocks than across trials within a given block. This inability to randomly re-assign motion directions to individual dots on a trial-by-trial basis most likely reduces the quality of kernel estimates in the logistic regression approach, since the variance in the motion signal is artificially reduced. In extreme cases, different responses (false alarms or correct rejections, respectively) are correlated with stimuli containing identical motion vectors. This reduced variation in the motion signal could be one reason why we failed to compute statistically reliable kernels for each subject separately. As it stands, our reverse correlation analysis did not reveal any evidence for changes in the overall shape of perceptual filters across intervals, which would be consistent with the absence of changes in sensory processing. However, the fact that we do not find these changes is mainly due to the lack of statistical power and the noise inherent in our kernel estimation process. Currently, we are repeating the experiment using an improved version of the software that allows a random re-assignment of motion directions for every single trial. Note however, that despite our problems of incomplete randomization, we were still able to extract meaningful estimates of the subjects' kernels (Table 1), from which one can readily extract, e.g., the designated target signal direction (Fig. 3a,b).

In summary, we have devised a discrete-interval signal detection paradigm that allows us to disentangle alternative mechanisms explaining behavioral benefits of successful anticipation of upcoming signals. Applying methods of signal detection theory we demonstrate that behavioral benefits of signal anticipation are not mediated by changes in sensory processing but rather result from adjustments in decision-related variables. As a complementary approach, we have used behavioral reverse correlation analysis to estimate perceptual filters of human observers, and have examined whether predictability of upcoming signals can lead to changes in these filters. Although the results from these latter analyses tend to support the findings obtained with signal detection theory, we cannot make firm conclusions about the absence of significant changes in the perceptual filters yet.

\section{Methods}

Subjects. 8 subjects (ages 21-28, 2 males and 6 females) participated in this study. All had 
normal or corrected-to-normal vision. They gave informed written consent and were naïve as to the purpose of the experiment.

Stimulus and apparatus. The stimulus was composed of dots moving within a virtual circular aperture of $5^{\circ}$ in diameter. A total of 250 dots was presented within this aperture. Each dot subtended $0.075^{\circ}$ of visual angle. All the dots were black and the RDP was plotted at the center of gaze, against a white background. All dots moved at a speed of $5^{\circ} / \mathrm{s}$. A noise stimulus was characterized by all dots moving in random directions ( $0 \%$ coherence). In contrast, a target motion signal exhibited some threshold level of coherent motion, which was embedded in the noise. The stimulus was presented on a VGA monitor (Lacie, Electron22 Blue IV) operating at a refresh rate of $85 \mathrm{~Hz}$ and a resolution of 80 pixels per degree of visual angle. Presentation of the stimulus and recording of the responses was controlled by an Apple Power Mac G4 computer.

Design and procedure. At trial start, a stationary dot pattern of $235 \mathrm{~ms}$ duration was presented as a warning signal. Following this, subjects viewed a temporal sequence of $1-7$ brief presentations of a centrally displayed, moving random dot pattern $(235 \mathrm{~ms})$, always followed by a blank screen (1500 ms). The subject's task was to press a button upon detection of a threshold-level coherent motion signal of a pre-defined direction, embedded in a sequence of otherwise random noise stimuli ( $0 \%$ coherence). For half of the subjects, the target signal consisted of rightward motion, for the other half leftward motion was used. Trials were terminated after a subject's response (hit or false alarm), but also after the target had been presented and no response was given before the next stimulus in the sequence would have been presented (miss). Subjects received auditory feedback after each trial. An increasing hazard rate was realized by drawing the target-interval for a given trial from a uniform distribution defined over the values $1-7$. A constant hazard rate was achieved by drawing the target-interval from a geometric, i.e. non-aging, distribution $($ mean $=4)$. In case the draw from the geometric distribution produced a number larger than 7 , no target signal was presented and the trial was considered a no-go. The experiment was divided into 6 sessions, each consisting of 3 blocks, that were performed on consecutive days. For one half of the subjects, the hazard rate was kept constant for the first nine blocks, and remained increasing for the second nine blocks. For the second half of the subjects, this assignment was reversed. Subjects were neither informed about the different signal interval distributions nor about the change in hazard conditions. For each subject and before each single block, a standard two-interval forced-choice staircase algorithm (Kaernbach, 1991) was run to determine the individual, practice-dependent level of coherent motion signal that was required to achieve $75 \%$ correct responses. This current estimation of individual threshold-level motion coherence was then used for the subsequent experimental block. Such a single experimental block was composed of 53 trials. The first three trials were used by the subjects to get acquainted with the strength of the target signal and were not included in any of the analyses.

Data analysis. One subject was excluded from the analyses because he did not produce any false alarms in the sixth interval. In such a case, meaningful signal detection measures $\left(d^{\prime}\right.$ and $\beta$ ) cannot be computed. All analyses were performed using Matlab (The MathWorks, Inc.) 
and the R environment for statistical computing (R Development Core Team, 2006).

Signal detection analysis. We used signal detection theory (Green and Swets, 1966) to compute measures for perceptual sensitivity $\left(d^{\prime}\right)$ and response criterion $(\beta)$. These two measures were determined for every combination of subject, signal interval, and hazard condition. Statistical significance was evaluated with a two-way Analysis of Variance (ANOVA), separately for $d^{\prime}$ and $\beta$, involving the within-subject factors hazard condition (increasing vs. constant) and stimulus interval (1-6). Interval 7 was excluded from analysis since, in the case of an increasing hazard rate, no false alarms can be generated in this interval. The Greenhouse-Geyser correction has been applied where appropriate.

Reverse correlation analysis. Following Cook and Maunsell (2004), we assumed that an subject's response $(R)$ is the result of a perceptual filter $(K)$ applied to the motion signal in the stimulus $(M)$, which can be expressed as a convolution

$$
R=K * M
$$

The convolution in Equation 1 can also be formulated as a linear regression problem (Ahumada Jr. and Lovell, 1971). Since we regress motion components on binary response outcomes, i.e. false alarms (1) or correct rejections (0), we used multiple logistic regression to estimate the kernel weights. The logistic regression model is a special case of the general linear model, in which the link function consists of the $\operatorname{logit}$ transformation $\log [p /(1-p)]$, symbolized by $\operatorname{logit}(p)$ (Agresti, 1996). Positive values for estimated coefficients reflect an increased, negative coefficients a decreased probability for an outcome of 1 , respectively. Thus, our regression model is given by

$$
\operatorname{logit}\left(p_{i}\right)=\log \left(\frac{p_{i}}{1-p_{i}}\right)=c+\sum_{d=1}^{12} k_{i, d} m_{i, d}
$$

where $c$ corresponds to the intercept term, $i$ is an index for interval, $d$ an index for direction of motion, $k$ represents the kernel weights, and $m$ the strength of the direction signal in the random noise stimulus. Using a bin size of $30^{\circ}$, Equation 2 expands to

$$
\operatorname{logit}\left(p_{i}\right)=c+k_{i, 15} m_{i, 15}+k_{i, 45} m_{i, 45}+\ldots+k_{i, 345} m_{i, 345}
$$

The obtained kernel weights as determined by the regression analysis are independent. However, since adjacent kernel weights are correlated, we have smoothed the kernel weights with a Gaussian function having a sigma of 1 bin $\left(30^{\circ}\right)$. To compute kernels across subjects, we have horizontally flipped the kernels for those subjects who had been assigned leftward motion as the target direction.

To test for differences in shape between kernels in successive intervals we adapted the procedure proposed by Cook and Maunsell (2004). First, we determined a best-fit scaling factor by which the kernel weights for a given interval $(i)$ would have to be multiplied to 
obtain the corresponding kernel weights for the subsequent interval $(i+1)$. To do so, we minimized a $\chi^{2}$-merit function with respect to the scaling factor $\gamma$ (Press et al., 1992):

$$
\chi^{2}=\sum_{d=1}^{12} \frac{\left(\gamma k_{i_{d}}-k_{i+1_{d}}\right)^{2}}{\gamma^{2} \sigma_{\text {noise }_{i}}^{2}+\sigma_{\text {noise }_{i+1}}^{2}}
$$

In Equation 4, the denominator represents the weighted sum of variances for 'noise kernels' $\left(\sigma_{\text {noise }}^{2}\right)$ in two successive intervals $i$. We computed these 'noise kernels' by correlating behavioral responses with such 0\%-coherence stimuli that have never been presented (because the trial had ended before that particular stimulus was going to be shown; see, e.g., the last two stimuli in Fig. 1), or that were going to be presented in future trials, relative to the current behavioral response (Fig. 3 b).

Second, we predicted kernel weights for interval $i+1$ by multiplying the kernel for interval $i$ with the estimated scaling factor. Such predictions will never be perfect, though, and the resulting residual kernels $K_{\text {res }}$ are given by the difference between the predicted kernel weights and the actual kernel weights for interval $i+1$.

$$
K_{\text {res }}=\gamma K_{i}-K_{i+1}
$$

Non-zero weights in the residual kernel, however, not only reflect deviations from multiplicative scaling, but also arise because of noise inherent in the estimation procedure. To disentangle these two components, we predicted the variance of $K_{\text {res }}$ due to noise $\left(\sigma_{0}\right)$ by the weighted sum of the variances in pairs of successive noise kernels.

$$
\sigma_{0}=\gamma^{2} \sigma_{\text {noise }_{i}}^{2}+\sigma_{\text {noise }_{i+1}}^{2}
$$

where $\gamma$ is our estimated multiplicative scaling factor. Deviations from multiplicative scaling would result in a larger variance in the residual kernel $K_{\text {res }}$ compared to the variance predicted on the basis of noise kernels $\left(\sigma_{0}\right)$. Statistical significance can then be evaluated by

$$
L=\frac{v \sigma_{r e s}^{2}}{\sigma_{0}^{2}}
$$

where $L$ follows a $\chi^{2}$-distribution with $v=12-1$ degrees of freedom (Zar, 1999).

Finally, we used a bootstrap approach to test whether the peak-to-peak amplitudes increased as a function of interval for the two hazard conditions. We first determined, for each interval separately, the difference between the maximal and minimal kernel weights and computed the slope of the best-fit line through the amplitude values. To test whether the slope of this line is significantly different from zero, we created bootstrap replicates of kernels by sampling with replacement the weights for each motion direction randomly from different intervals. We then smoothed the bootstrap replicates of the kernels with a Gaussian filter (sigma $=1 \mathrm{bin}$ ), before determining the slopes of the best-fit line through the amplitude values. We repeated this procedure 10000 times to obtain a distribution of bootstrapped slopes, which in 
turn allowed us to determine statistical significance of the actual slope. This procedure was done separately for increasing and constant hazard conditions.

\section{References}

Agresti, A. (1996). An introduction to categorical data analysis. Wiley, New York.

Ahumada Jr., A. J. and Lovell, J. (1971). Stimulus features in signal detection. Journal of the Acoustical Society of America, 49(6):1751-1756.

Barlow, H. and Tripathy, S. P. (1997). Correspondence noise and signal pooling in the detection of coherent visual motion. Journal of Neuroscience, 17(20):7954-7966.

Bertelson, P. and Tisseyre, F. (1968). The time-course of preparation with regular and irregular foreperiods. Quarterly Journal of Experimental Psychology, 20(3):297-300.

Blake, D. T. and Merzenich, M. M. (2002). Changes of AI receptive fields with sound density. Journal of Neurophysiology, 88(6):3409-3420.

Cook, E. P. and Maunsell, J. H. (2004). Attentional modulation of motion integration of individual neurons in the middle temporal visual area. Journal of Neuroscience, 24(36):79647977.

DiCarlo, J. J., Johnson, K. O., and Hsiao, S. S. (1998). Structure of receptive fields in area 3b of primary somatosensory cortex in the alert monkey. Journal of Neuroscience, 18(7):26262645.

Drazin, D. H. (1961). Effects of foreperiod, foreperiod variability, and probability of stimulus occurrence on simple reaction time. Journal of Experimental Psychology, 62:43-50.

Eckstein, M. P., Shimozaki, S. S., and Abbey, C. K. (2002). The footprints of visual attention in the Posner cueing paradigm revealed by classification images. Journal of Vision, 2(1):2545.

Efron, B. and Tibshirani, R. (1993). An Introduction to the Bootstrap. Chapman and Hall, London.

Firth, D. (1993). Bias reduction of maximum likelihood estimates. Biometrika, 80(1):27-38.

Ghose, G. M. and Maunsell, J. H. (2002). Attentional modulation in visual cortex depends on task timing. Nature, 419(6907):616-620.

Green, D. M. and Swets, J. A. (1966). Signal detection theory and psychophysics. Wiley, New York. 
Hanks, T. D., Ditterich, J., and Shadlen, M. N. (2006). Microstimulation of macaque area LIP affects decision-making in a motion discrimination task. Nature Neuroscience, 9(5):682-689.

Janssen, P. and Shadlen, M. N. (2005). A representation of the hazard rate of elapsed time in macaque area LIP. Nature Neuroscience, 8(2):234-241.

Kaernbach, C. (1991). Simple adaptive testing with the weighted up-down method. Perception \&5 Psychophysics, 49(3):227-229.

Klemmer, E. T. (1956). Time uncertainty in simple reaction time. Journal of Experimental Psychology, 51(3):179-184.

Neri, P. and Levi, D. M. (2006). Receptive versus perceptive fields from the reverse-correlation viewpoint. Vision Research, 46(16):2465-2474.

Nickerson, R. S. and Burnham, D. W. (1969). Response times with nonaging foreperiods. Journal of Experimental Psychology, 79(3):452-457.

Niemi, P. and Näätänen, R. (1981). Foreperiod and simple reaction time. Psychological Bulletin, 89(1):133-162.

Platt, M. L. and Glimcher, P. W. (1999). Neural correlates of decision variables in parietal cortex. Nature, 400(6741):233-238.

Press, W. H., Teukolsky, S. A., Vetterling, W. T., and Flannery, B. P. (1992). Numerical Recipes in C: the Art of Scientific Computing. Cambridge University Press, Cambridge, UK, 2nd edition.

R Development Core Team (2006). R: A Language and Environment for Statistical Computing. R Foundation for Statistical Computing, Vienna, Austria. ISBN 3-900051-07-0.

Requin, J. and Granjon, M. (1969). The effect of conditional probability of the response signal on the simple reaction time. Acta Psychologica, 31(2):129-144.

Ringach, D. and Shapley, R. (2004). Reverse correlation in neurophysiology. Cognitive Science, 28:147-166.

Sakai, H. M., Naka, K., and Korenberg, M. J. (1988). White-noise analysis in visual neuroscience. Visual Neuroscience, 1(3):287-296.

Theunissen, F. E., David, S. V., Singh, N. C., Hsu, A., Vinje, W. E., and Gallant, J. L. (2001). Estimating spatio-temporal receptive fields of auditory and visual neurons from their responses to natural stimuli. Network, 12(3):289-316.

Zar, J. H. (1999). Biostatistical Analysis. Prentice Hall, Upper Saddle River, NJ, 4th edition. 


\section{Chapter 3}

\section{Summary}

In summary, this thesis investigates cognitive influences on the processing of visual motion information. A major part of this work focuses on the time course of attentional modulation during shifts of attention. Using extracellular single-unit recordings in the motion sensitive area MT of awake behaving macaque monkeys, we show that automatic and voluntary shifts of attention are associated with characteristic modulations of sensory information processing. In a complementary behavioral experiment, we investigate spatial and feature-based attentional effects of exogenous cueing on the processing of visual motion. Finally, a short review summarizes one of the first electrophysiological studies directly measuring the temporal dynamics of neuronal modulation in area V1 during shifts of attention.

A second focus of this thesis are the attentional influences on the processing of color and motion. We provide the first single-unit evidence for object-based attentional modulation in the primate visual cortex showing that attention to the color of a moving object enhances neuronal activity in the motion-sensitive area MT. A concomitant behavioral experiment shows that feature-based attentional mechanisms can best explain the perceptual integration of color and motion information in a divided attention paradigm.

The remaining research articles investigate the effects of attention on perceptual tuning curves

for direction of motion, and the influences of signal anticipation on perceptual sensitivity and response criterion. Together, these contributions show that cognitive factors strongly modulate the processing of visual motion. 


\section{Bibliography}

Adelson, E. H. and Bergen, J. R. (1985). Spatiotemporal energy models for the perception of motion. Journal of the Optical Society of America A, 2(2):284-299.

Adelson, E. H. and Movshon, J. A. (1982). Phenomenal coherence of moving visual patterns. Nature, 300(5892):523-525.

Albright, T. D. (1984). Direction and orientation selectivity of neurons in visual area MT of the macaque. Journal of Neurophysiology, 52(6):1106-1130.

Albright, T. D., Desimone, R., and Gross, C. G. (1984). Columnar organization of directionally selective cells in visual area MT of the macaque. Journal of Neurophysiology, 51(1):16-31.

Borghuis, B. G., Perge, J. A., Vajda, I., van Wezel, R. J., van de Grind, W. A., and Lankheet, M. J. (2003). The motion reverse correlation (MRC) method: a linear systems approach in the motion domain. Journal of Neuroscience Methods, 123(2):153-166.

Born, R. T. and Bradley, D. C. (2005). Structure and function of visual area MT. Annual Review of Neuroscience, 28:157-189.

Boynton, G. M., Ciaramitaro, V. M., and Arman, A. C. (2006). Effects of feature-based attention on the motion aftereffect at remote locations. Vision Research, 46(18):2968-2976.

Brefczynski, J. A. and DeYoe, E. A. (1999). A physiological correlate of the 'spotlight' of visual attention. Nature Neuroscience, 2(4):370-374.

Britten, K. H. (2004). The middle temporal area: motion processing and the link to perception. In Chalupa, L. M. and Werner, J. F., editors, The Visual Neurosciences, pages 1203-1216. MIT Press, Cambridge, MA.

Britten, K. H., Newsome, W. T., Shadlen, M. N., Celebrini, S., and Movshon, J. A. (1996). A relationship between behavioral choice and the visual responses of neurons in macaque MT. Visual Neuroscience, 13(1):87-100. 
Britten, K. H., Shadlen, M. N., Newsome, W. T., and Movshon, J. A. (1992). The analysis of visual motion: a comparison of neuronal and psychophysical performance. Journal of Neuroscience, 12(12):4745-4765.

Chichilnisky, E. J. (2001). A simple white noise analysis of neuronal light responses. Network: Computation in Neural Systems, 12(2):199-213.

Compte, A. and Wang, X.-J. (2006). Tuning curve shift by attention modulation in cortical neurons: a computational study of its mechanisms. Cerebral Cortex, 16(6):761-778.

Connor, C. E., Preddie, D. C., Gallant, J. L., and Van Essen, D. C. (1997). Spatial attention effects in macaque area V4. Journal of Neuroscience, 17(9):3201-3214.

Cook, E. P. and Maunsell, J. H. (2002). Dynamics of neuronal responses in macaque MT and VIP during motion detection. Nature Neuroscience, 5(10):985-994.

Culham, J., He, S., Dukelow, S., and Verstraten, F. A. (2001). Visual motion and the human brain: what has neuroimaging told us? Acta Psychologica, 107(1-3):69-94.

Dayan, P. and Abbott, L. F. (2001). Theoretical Neuroscience. Computational and Mathematical Modeling of Neural Systems, chapter Neural encoding II: Reverse correlation and visual receptive fields, pages 45-85. MIT Press.

DeAngelis, G. C., Cumming, B. G., and Newsome, W. T. (1998). Cortical area mt and the perception of stereoscopic depth. Nature, 394(6694):677-680.

DeAngelis, G. C. and Newsome, W. T. (1999). Organization of disparity-selective neurons in macaque area MT. Journal of Neuroscience, 19(4):1398-1415.

DeAngelis, G. C., Ohzawa, I., and Freeman, R. D. (1995). Receptive-field dynamics in the central visual pathways. Trends in Neurosciences, 18:451-458.

Desimone, R. and Duncan, J. (1995). Neural mechanisms of selective visual attention. Annual Review of Neuroscience, 18:193-222.

Dubner, R. and Zeki, S. M. (1971). Response properties and receptive fields of cells in an anatomically defined region of the superior temporal sulcus in the monkey. Brain Research, 35:528-532.

Duncan, J. (1984). Selective attention and the organization of visual information. Journal of Experimental Psychology: General, 113(4):501-517. 
Egly, R., Driver, J., and Rafal, R. D. (1994). Shifting visual attention between objects and locations: evidence for normal and parietal lesion subjects. Journal of Experimental Psychology: General, 123(2):161-177.

Felleman, D. J. and Van Essen, D. C. (1991). Distributed hierarchical processing in the primate cerebral cortex. Cerebral Cortex, 1(1):1-47.

Frechette, E. S., Sher, A., Grivich, M. I., Petrusca, D., Litke, A. M., and Chichilnisky, E. J. (2005). Fidelity of the ensemble code for visual motion in primate retina. Journal of Neurophysiology, 94(1):119-135.

Fries, P., Reynolds, J. H., Rorie, A. E., and Desimone, R. (2001). Modulation of oscillatory neuronal synchronization by selective visual attention. Science, 291(5508):1560-1563.

Gattass, R. and Gross, C. G. (1981). Visual topography of striate projection zone (MT) in posterior superior temporal sulcus of the macaque. Journal of Neurophysiology, 46(3):621-638.

Geesaman, B. J., Born, R. T., Andersen, R. A., and Tootell, R. B. (1997). Maps of complex motion selectivity in the superior temporal cortex of the alert macaque monkey: a doublelabel 2-deoxyglucose study. Cerebral Cortex, 7(8):749-757.

Ghose, G. M. and Maunsell, J. H. (2002). Attentional modulation in visual cortex depends on task timing. Nature, 419(6907):616-620.

Guo, K., Benson, P. J., and Blakemore, C. (2004). Pattern motion is present in V1 of awake but not anaesthetized monkeys. European Journal of Neuroscience, 19(4):1055-1066.

Hawken, M. J., Parker, A. J., and Lund, J. S. (1988). Laminar organization and contrast sensitivity of direction-selective cells in the striate cortex of the old world monkey. Journal of Neuroscience, 8(10):3541-3548.

Hubel, D. H. and Wiesel, T. N. (1968). Receptive fields and functional architecture of monkey striate cortex. Journal of Physiology, 195(1):215-243.

Janssen, P. and Shadlen, M. N. (2005). A representation of the hazard rate of elapsed time in macaque area LIP. Nature Neuroscience, 8(2):234-241.

Kastner, S. and Ungerleider, L. G. (2000). Mechanisms of visual attention in the human cortex. Annual Review of Neuroscience, 23:315-341. 
Khayat, P. S., Spekreijse, H., and Roelfsema, P. R. (2006). Attention lights up new object representations before the old ones fade away. Journal of Neuroscience, 26(1):138-142.

Klein, R. M. (2000). Inhibition of return. Trends in Cognitive Sciences, 4(4):138-147.

Lauwers, K., Saunders, R., Vogels, R., Vandenbussche, E., and Orban, G. A. (2000). Impairment in motion discrimination tasks is unrelated to amount of damage to superior temporal sulcus motion areas. The Journal of Comparative Neurology, 420(4):539-557.

Liu, J. and Newsome, W. T. (2003). Functional organization of speed tuned neurons in visual area MT. Journal of Neurophysiology, 89(1):246-256.

Liu, J. and Newsome, W. T. (2005). Correlation between speed perception and neural activity in the middle temporal visual area. Journal of Neuroscience, 25(3):711-722.

Luck, S. J., Chelazzi, L., Hillyard, S. A., and Desimone, R. (1997). Neural mechanisms of spatial selective attention in areas V1, V2, and V4 of macaque visual cortex. Journal of Neurophysiology, 77(1):24-42.

Martínez-Trujillo, J. and Treue, S. (2002). Attentional modulation strength in cortical area MT depends on stimulus contrast. Neuron, 35(2):365-370.

Martínez-Trujillo, J. C. and Treue, S. (2004). Feature-based attention increases the selectivity of population responses in primate visual cortex. Current Biology, 14(9):744-751.

Maunsell, J. H. and Van Essen, D. C. (1983). Functional properties of neurons in middle temporal visual area of the macaque monkey. I. Selectivity for stimulus direction, speed, and orientation. Journal of Neurophysiology, 49(5):1127-1147.

Maunsell, J. H. R. and Treue, S. (2006). Feature-based attention in visual cortex. Trends in Neurosciences, 29(6):317-322.

McAdams, C. J. and Maunsell, J. H. (1999). Effects of attention on orientation-tuning functions of single neurons in macaque cortical area V4. Journal of Neuroscience, 19(1):431-441.

McAlonan, K., Cavanaugh, J., and Wurtz, R. H. (2006). Attentional modulation of thalamic reticular neurons. Journal of Neuroscience, 26(16):4444-4450.

Melcher, D., Papathomas, T. V., and Vidnyanszky, Z. (2005). Implicit attentional selection of bound visual features. Neuron, 46(5):723-729. 
Moran, J. and Desimone, R. (1985). Selective attention gates visual processing in the extrastriate cortex. Science, 229(4715):782-784.

Motter, B. C. (1994). Neural correlates of feature selective memory and pop-out in extrastriate area V4. Journal of Neuroscience, 4(4):2190-2199.

Movshon, J. A., Adelson, E. H., Gizzi, M. S., and Newsome, W. T. (1985). The analysis of moving visual patterns. In Chagas, C., Gattass, R., and Gross, C., editors, Study Group of Pattern Recognition Mechanisms, pages 117-151. Pontifica Academia Scientiarum, Vatican City.

Müller, M. M., Teder-Sälejärvi, W., and Hillyard, S. A. (1998). The time course of cortical facilitation during cued shifts of spatial attention. Nature Neuroscience, 1(7):631-634.

Müller, N. G. and Kleinschmidt, A. (2003). Dynamic interaction of object- and space-based attention in retinotopic visual areas. Journal of Neuroscience, 23(30):9812-9816.

Newsome, W. T., Britten, K. H., and Movshon, J. A. (1989). Neuronal correlates of a perceptual decision. Nature, 341(6237):52-54.

Newsome, W. T. and Pare, E. B. (1988). A selective impairment of motion perception following lesions of the middle temporal visual area (MT). Journal of Neuroscience, 8(6):2201-2211.

Niemi, P. and Näätänen, R. (1981). Foreperiod and simple reaction time. Psychological Bulletin, 89(1):133-162.

O’Connor, D. H., Fukui, M. M., Pinsk, M. A., and Kastner, S. (2002). Attention modulates responses in the human lateral geniculate nucleus. Nature Neuroscience, 5(11):1203-1209.

O'Craven, K. M., Downing, P. E., and Kanwisher, N. (1999). fMRI evidence for objects as the units of attentional selection. Nature, 401(6753):584-587.

Orban, G. A., Saunders, R. C., and Vandenbussche, E. (1995). Lesions of the superior temporal cortical motion areas impair speed discrimination in the macaque monkey. European Journal of Neuroscience, 7(11):2261-2276.

Parker, A. J. and Newsome, W. T. (1998). Sense and the single neuron: probing the physiology of perception. Annual Review of Neuroscience, 21:227-277.

Pasternak, T. and Merigan, W. H. (1994). Motion perception following lesions of the superior temporal sulcus in the monkey. Cerebral Cortex, 4(3):247-259. 
Perge, J. A., Borghuis, B. G., Bours, R. J., Lankheet, M. J., and van Wezel, R. J. (2005). Temporal dynamics of direction tuning in motion-sensitive macaque area MT. Journal of Neurophysiology, 93(4):2104-2116.

Posner, M. I. (1980). Orienting of attention. Quarterly Journal of Experimental Psychology, $32(1): 3-25$.

Posner, M. I. and Cohen, Y. (1984). Components of visual orienting. In Bouma, H. and Bouwhuis, D. G., editors, Attention and Performance X, pages 531-556. Erlbaum, Hillsdale, NJ.

Purushothaman, G. and Bradley, D. C. (2005). Neural population code for fine perceptual decisions in area mt. Nature Neuroscience, 8(1):99-106.

Reynolds, J. H., Pasternak, T., and Desimone, R. (2000). Attention increases sensitivity of V4 neurons. Neuron, 26:703-714.

Rizzolatti, G., Riggio, L., Dascola, I., and Umilta, C. (1987). Reorienting attention across the horizontal and vertical meridians: evidence in favor of a premotor theory of attention. Neuropsychologia, 25(1A):31-40.

Roelfsema, P. R., Lamme, V. A., and Spekreijse, H. (1998). Object-based attention in the primary visual cortex of the macaque monkey. Nature, 395(6700):376-381.

Sàenz, M., Buraĉas, G. T., and Boynton, G. M. (2002). Global effects of feature-based attention in human visual cortex. Nature Neuroscience, 5(7):631-632.

Sàenz, M., Buraĉas, G. T., and Boynton, G. M. (2003). Global feature-based attention for motion and color. Vision Research, 43(6):629-637.

Salzman, C. D., Britten, K. H., and Newsome, W. T. (1990). Cortical microstimulation influences perceptual judgements of motion direction. Nature, 346(6280):174-177.

Schiller, P. H. (1993). The effects of V4 and middle temporal (MT) area lesions on visual performance in the rhesus monkey. Visual Neuroscience, 10(4):717-746.

Shadlen, M. N., Britten, K. H., Newsome, W. T., and Movshon, J. A. (1996). A computational analysis of the relationship between neuronal and behavioral responses to visual motion. Journal of Neuroscience, 16(4):1486-1510. 
Simoncelli, E. P. and Heeger, D. J. (1998). A model of neuronal responses in visual area mt. Vision Research, 38(5):743-761.

Snowden, R. J., Treue, S., and Andersen, R. A. (1992). The response of neurons in areas V1 and MT of the alert rhesus monkey to moving random dot patterns. Experimental Brain Research, 88(2):389-400.

Tanner, W. P. and Swets, J. A. (1954). A decision-making theory of visual detection. Psychological Review, 61(6):401-409.

Theeuwes, J., Godijn, R., and Pratt, J. (2004). A new estimation of the duration of attentional dwell time. Psychonomic Bulletin \& Review, 11(1):60-64.

Tinsley, C. J., Webb, B. S., Barraclough, N. E., Vincent, C. J., Parker, A., and Derrington, A. M. (2003). The nature of V1 neural responses to 2D moving patterns depends on receptive-field structure in the marmoset monkey. Journal of Neurophysiology, 90(2):930-937.

Treisman, A. M. and Gelade, G. (1980). A feature-integration theory of attention. Cognitive Psychology, 12(1):97-136.

Treue, S. (2001). Neural correlates of attention in primate visual cortex. Trends in Neurosciences, 24(5):295-300.

Treue, S. and Martínez-Trujillo, J. C. (1999). Feature-based attention influences motion processing gain in macaque visual cortex. Nature, 399(6736):575-579.

Treue, S. and Maunsell, J. H. R. (1996). Attentional modulation of visual motion processing in cortical areas MT and MST. Nature, 382(6591):539-541.

Ungerleider, L. G. and Mishkin, M. (1982). Two cortical visual systems. In Ingle, D. J., Goodale, M. A., and Mansfield, R. J. W., editors, Analysis of Visual Behavior, pages 549-586. MIT Press, Cambridge, MA.

Van Essen, D. C., Maunsell, J. H. R., and Bixby, J. L. (1981). The middle temporal visual area in the macaque: myeloarchitecture, connections, functional properties and topographic organization. Journal of Comparative Neurology, 199(3):293-326.

Wandell, B. A. (1995). Foundations of Vision, chapter 10. Motion and Depth, pages 341-385. Sinauer Associates, Inc., Sunderland, Massachusetts. 
Watson, A. B. and Ahumada Jr., A. J. (1983). A look at motion in the frequency domain. NASA Technical Memorandum 84352.

Williford, T. and Maunsell, J. H. R. (2006). Effects of spatial attention on contrast response functions in macaque area v4. Journal of Neurophysiology, 96(1):40-54.

Wolfe, J. M. (1994). Guided search 2.0: a revised model of visual search. Psychonomic Bulletin \& Review, 1:202-238.

Wolfe, J. M. (1998). What can 1 million trials tell us about visual search? Psychological Science, 9(1):33-39.

Womelsdorf, T., Anton-Erxleben, K., Pieper, F., and Treue, S. (2006). Dynamic shifts of visual receptive fields in cortical area MT by spatial attention. Nature Neuroscience, advanced online publication.

Zeki, S., Watson, J. D., Lueck, C. J., Friston, K. J., Kennard, C., and Frackowiak, R. S. (1991). A direct demonstration of functional specialization in human visual cortex. Journal of Neuroscience, 11(3):641-649. 


\section{Curriculum Vitae}

\section{Laura Busse}

\section{Scientific Education}

since 12/02 Graduate student, German Primate Center, Göttingen, Germany

Advisor: Prof. Dr. Stefan Treue

10/99-09/01 M.Sc. Neural \& Behavioral Sciences, University of Tübingen, Germany

International Max-Planck Research School

Thesis: The ERP Omitted Stimulus Response to 'No-Stim' events and its implications for fast-rate event-related fMRI designs.

Advisor: Prof. Dr. Marty G. Woldorff

10/97-09/99 Undergraduate studies in Psychology, University of Leipzig, Germany

06/97 Abitur, Spessart-Gymnasium Alzenau, Germany

\section{Relevant Experience}

$06 / 06$

Participant in the Summer Course "Computational Neuroscience: Vision" at the Cold Spring Harbor Laboratories, Cold Spring Harbor, NY, USA

10/01-11/02 Research Assistant

Center for Cognitive Neuroscience, Duke University, Durham, NC, USA

Advisor: Prof. Dr. Marty G. Woldorff

02/01-07/01 Visiting Student

Center for Cognitive Neuroscience, Duke University, Durham, NC, USA

Advisor: Prof. Dr. Marty G. Woldorff 
09/98-09/99 Research Assistant (part-time)

Max-Planck Institute for Cognitive Neuroscience, Leipzig, Germany

Advisor: Dr. Evelyn C. Ferstl \& Dr. Katja Werheid

\section{Scholarships}

10/97-09/01 Scholarship by the Bavarian State Ministry

\section{Journal publications}
- $\quad$ Busse, L. and Katzner, S. (2006). The time course of shifting visual atten- tion. Journal of Neuroscience, 26(15):3885-3886.
Katzner, S., Busse, L., and Treue, S. (2006). Feature-based attentional inte- gration of color and visual motion. Journal of Vision, 6(3):269-284.
Busse, L., Katzner, S., and Treue, S. (2006). Spatial and feature-based ef- fects of exogenous cueing on visual motion processing. Vision Research, 46(13):2019-2027.

Schmajuk, M., Liotti, M., Busse, L., and Woldorff, M. G. (2006). Electrophysiological activity underlying inhibitory control processes in normal adults. Neuropsychologia, 44(3):384-395.

Busse, L., Roberts, K. C., Crist, R. E., Weissman, D. H., and Woldorff, M. G. (2005). The spread of attention across modalities and space in a multisensory object. Proceedings of the National Academy of Sciences, 102(51):1875118756.

Busse, L. and Woldorff, M. G. (2003). The ERP omitted stimulus response to "no-stim" events and its implications for fast-rate event-related fMRI designs. NeuroImage, 18(4):856-864.

Woldorff, M. G., Liotti, M., Seabolt, M., Busse, L., Lancaster, J. L., and Fox, P. T. (2002). The temporal dynamics of the effects in occipital cortex of visual-spatial selective attention. Cognitive Brain Research, 15(1):1-15.

Lutzenberger, W., Ripper, B., Busse, L., Birbaumer, N., and Kaiser, J. (2002). Dynamics of gamma-band activity during an audiospatial working memory task in humans. Journal of Neuroscience, 22(13):5630-5638. 


\title{
Published abstracts
}

\author{
- $\quad$ Busse, L., Katzner, S. \& Treue, S. (2006). The time course of spatial atten- \\ tion effects in macaque area MT. Poster at the meeting of the Society for \\ Neuroscience, Atlanta, GA. \\ Katzner, S. Busse, L., Tillmann, C. \& Treue, S. (2006). Effects of attention \\ on perceptual tuning curves for direction of motion. Poster at the meeting of \\ the Society for Neuroscience, Atlanta, GA. \\ Tillmann, C., Busse, L., Katzner, S. \& Treue, S. (2006). Attentional modu- \\ lation of psychophysical tuning curves for direction of motion. Poster pre- \\ sented at the European Conference on Visual Perception, St. Petersburg, \\ Russia.
}

Busse, L., Katzner, S. \& Treue, S. (2005). Temporal dynamics of attentional modulation of neuronal responses in macaque area MT. Talk presented at the meeting of the Society for Neuroscience, Washington, DC.

Katzner, S., Busse, L. \& Treue, S. (2005). Attending to the color in a motion signal: Evidence for object-based attentional effects in macaque area MT. Talk presented at the meeting of the Society for Neuroscience, Washington, DC.

Treue, S., Busse, L. \& Katzner, S. (2005). Behavioral benefits of signal anticipation in motion detection: improvement in perceptual sensitivity or adjustment of decision criterion? Poster presented at the meeting of the Society for Neuroscience, Washington, DC.

Busse, L., Katzner, S., Real, R. G. \& Treue, S. (2004). Time course of shifts of covert attention across features and space. Poster presented at the meeting of the Society for Neuroscience, San Diego.

Katzner, S., Busse, L. \& Treue, S. (2004). Dividing attention between color and motion: Feature-based effects of redundant targets. Poster presented at the meeting of the Society for Neuroscience, San Diego.

Busse, L., Katzner, S. \& Treue, S. (2004). The effects of exogenous attention on the processing of visual motion. Poster presented at the meeting of the 
Cognitive Neuroscience Society, San Francisco.
- $\quad$ Katzner, S. Busse, L., Akcay, C. \& Treue, S. (2004). Processing visual motion and color: The effect of redundant targets. Poster presented at the meeting of the Cognitive Neuroscience Society, San Francisco.
Busse, L., Katzner, S. \& Treue, S. (2003). Spatial and feature-based effects of exogenous cueing on visual motion processing. Poster presented at the meeting of the Society for Neuroscience, New Orleans.
Busse, L. \& Woldorff, M.G. (2003). Visual spatial attention modulates ERP brain responses to mislocated task-irrelevant tones in the ventriloquism illu- sion. Poster presented at the 10th Annual meeting of the Cognitive Neuro- science Society, New York, NJ.
Busse, L. \& Woldorff, M.G. (2002). Attention-related brain mechanisms underlying the auditory-visual perceptual grouping in the ventriloquism ef- fect. Talk presented at the 32nd meeting of the Society for Neuroscience, Orlando, FL.
Busse, L. \& Woldorff, M.G. (2002). The ERP Omitted Stimulus Response to No-Stim events and its implications for fast-rate event-related fMRI designs. Poster presented at the 9th Annual meeting of the Cognitive Neuroscience Society, San Francisco, CA.

Göttingen, 31.10.2006 